DOI: $10.1002 /$ adfm.201703523

Article type: Feature Article

\title{
Multifunctional Neural Interfaces for Closed-Loop Control of Neural Activity
}

Christopher A. R. Chapman, Noah Goshi, and Erkin Seker*

Dr. C. A. R. Chapman

Department of Medical Physics \& Biomedical Engineering

University College London

London, U.K. WC1E 6BT

N. Goshi

Department of Biomedical Engineering

University of California, Davis

Davis, CA 95616, USA

Prof. E. Seker

Department of Electrical and Computer Engineering

University of California, Davis

Davis, CA 95616, USA

E-mail: eseker@ucdavis.edu

Keywords: (neural interfaces, microfabrication, nanotechnology, high-throughput screening, closed-loop control)

Microfabrication and nanotechnology have significantly expanded the technological capabilities for monitoring and modulating neural activity with the goal of studying the nervous system and managing neurological disorders. This feature article initially provides a tutoriallike review of the prominent technologies for enabling this two-way communication with the nervous system via electrical, chemical, and optical means. Following this overview, the article discusses emerging high-throughput methods for identifying device attributes that enhance the functionality of interfaces. The discussion then extends into opportunities and challenges in integrating different device functions within a small footprint with the goal of closed-loop control of neural activity with high spatiotemporal resolution and reduced adverse tissue response. The article concludes with an outline of future directions in the development and applications of multifunctional neural interfaces. 


\section{WILEY-VCH}

\section{Introduction}

In properly functioning nervous tissue, neurons carry electrical signals, termed action potentials, through the tissue via a switching of the membrane potential caused by electricallyand chemically-gated ion channels embedded in the cell membrane. Although the action potential propagating along the length of the neuron is primarily achieved by electrical means, neurons communicate with each other at chemical junctions termed synapses via release and binding of various neurotransmitters. Through these complex chemical and electrical interactions between neurons as well as supporting cells (glia), information is distributed and processed in the central nervous system. In neurological disorders, spanning Alzheimer's to epilepsy, significant changes to this delicate neural tissue environment cause extremely deleterious effects ranging from memory loss to seizures. Whether this change is in electrical, chemical, or other nature, these disruptions possess valuable information for understanding and managing the disorders. Along with pharmaceutical and surgical modalities, the use of implanted devices to study and treat neurological conditions has been intriguing for both the academic and medical communities alike. One reason for this interest is that in order to successfully implement more effective treatments for dynamic disease states, such as epilepsy, it is necessary to achieve closed-loop control of the underlying physiological mechanisms of the disorder. More specifically, a successful device must be able to continuously monitor the state of the neural environment and respond by modulating it with an appropriate stimulus. For instance, as in the case of epilepsy, the device should keep track of anomalous electrophysiological signals and deliver stimuli to suppress a likely seizure ${ }^{[1]}$. This ability to both monitor and modulate the surrounding tissue via a single device, termed multifunctionality, has been a central goal of implanted devices for over a decade. To that end, there have been impressive advancements in multifunctional device engineering; however, challenges remain in combining multiple functions in an implantable device with a small form factor. A quick glance at the current state-of-the-art methods for monitoring and modulating neural activity 


\section{WILEY-VCH}

reveals that there are roughly five main approaches ${ }^{[2,3]}$ : electrical ${ }^{[4]}$, chemical $^{[5]}$, optical ${ }^{[6,7]}$, acoustic $^{[8,9]}$, and magnetic ${ }^{[10]}$. This review focuses specifically on the three modalities (electrical, chemical, and optical) that form a direct interface between device and neural tissue.

Electrical interfaces have been used extensively in neuroscience as tools to study neural electrophysiology and its manifestations in higher level activities, such as cognition and behavior. These interfaces typically consist of electrodes that interact with electrogenic cells that modulate their surrounding ionic environment (via action potentials). These collective ionic variations, known as field potentials, can be transduced into electrical signals via the electrodes or conversely the ionic environment can be influenced by injecting charge through the electrodes.

Chemical interfaces, in which biochemical species are either presented to or detected from the surrounding biological tissue, are another widely used tool for interacting with neural tissue. These interactions can range from simply presenting bioactive molecules on the surfaces of devices $^{[11]}$ to releasing such molecules from the device interface itself $f^{[12]}$. These interfaces can also monitor the neurochemical environment through direct electrochemical detection and microdialysis $^{[13,14]}$.

Optical interfaces are an emerging and transformative method primarily centered around optogenetic methods which are at present limited to non-human use ${ }^{[15]}$. For this approach, cells are genetically transformed to express light-sensitive ion channels, where optical stimulation at different wavelengths can excite or inhibit specific cell populations. Accompanying the optical stimulation front, development of new dyes that can transduce electrophysiological and neurochemical signals to optical information has created novel ways to monitor neural 


\section{WILEY-VCH}

activity $^{[16-19]}$. Both optical monitoring and modulation has demanded the fabrication of new interfaces to maximize the capabilities offered by these molecular approaches ${ }^{[15,20-23]}$.

Microfabrication technology has revolutionized the field of neural interfaces; however, as the need to integrate multiple functions on a single interface becomes more evident, miniaturization technology alone has proved to be insufficient. Innovations on the materials front, especially the emergence of new nanostructured materials with unique properties (e.g., mechanical, electrical, chemical, and optical ${ }^{[12,24-26]}$, have opened up new avenues to introduce multifunctional interfaces for closed-loop control of neural activity. The goal of this review is two-fold. We first provide an overview of the current state of miniaturization technology and nanostructured materials used in electrical, chemical, and optical monitoring and modulating interfaces. We then discuss the methods and challenges in combining these interfaces to achieve multifunctionality for closed-loop control of electrical, chemical, and optical neural devices.

\section{Monitoring Neural Activity}

\subsection{Electrical Monitoring}

Electrical interfaces are arguably the most widely utilized tools for studying the nervous system. Typically comprised of an electrode that interacts with surrounding electrogenic neural cells, the resulting interface formed between the cells and the electrode surface can be represented as an equivalent electrical circuit (Figure 1) that captures the essential system parameters. When action potentials propagate through a neuron via the opening of ion channels in the cell membrane (illustrated by purple lines in Figure 1) a local imbalance of charge is created. Combination of this time-varying ionic imbalance from numerous neurons, termed local field potential (denoted as $\mathrm{e}_{\mathrm{n}}$ in Figure 1), is then transduced by the neural electrical interface. This interface can then be broken down into approximately seven simplified electrical constituents. 


\section{WILEY-VCH}

Attributes such as physiological buffer resistance $\left(R_{s}\right)$, metal (trace) resistance $\left(R_{m}\right)$, shunt capacitance $\left(\mathrm{C}_{\mathrm{s}}\right)$ and amplifier impedance $\left(\mathrm{Z}_{\mathrm{a}}\right)$ can typically be ignored for a well-fabricated system. The other attributes, such as electrode material resistance $\left(\mathrm{R}_{\mathrm{e}}\right)$, and electrode doublelayer capacitance $\left(C_{e}\right)$ (which together can be represented as electrode impedance $\left(Z_{e}\right)$ ), alongside seal resistance $\left(\mathrm{R}_{\text {seal }}\right)$ largely dictate the nature of the interface. The reader is directed to comprehensive reviews on the neural-electrical interface for additional details ${ }^{[27-31]}$. For intracellular recording techniques, such as patch-clamp and other emerging approaches ${ }^{[28,32-34]}$, the electrical properties of the cell membrane and ionic channels become more important. However, for the sake of this review, we will mainly focus on extracellular recording approaches. In this section, we outline the utility of microfabrication and nanostructured materials in improving electrical monitoring of neural activity.

Dating as far back as the 1950 s, the monitoring neural activity initially via electrical interfaces involved wire electrodes (platinum or iridium) implanted into the brain ${ }^{[35]}$. These wires with typical diameters of 100 s of micrometers, consisted of a conducting metal core and an insulating sheath and enabled some of the first electrical recordings from neural tissue (Figure 2A) ${ }^{[35-38]}$. In order to sort the spike patterns from different neurons, it became necessary to have multiple recording sites, which was addressed by using tetrodes consisting of four thin wire electrodes (10s of micrometers in diameter) bundled together into a single implant (Figure 2B) ${ }^{[39]}$. These devices, easily accessible and manufacturable by researchers are still extensively used for electrophysiology; however, they nevertheless suffer from a very limited number of recording sites and consequently limited neural spiking information.

The emergence of microfabrication technology enabled the production of microelectrode arrays with arbitrary geometries patterned on a variety of substrates. These fabrication techniques constitute the foundation of the current state-of-the-art devices (e.g., Michigan-style planar 


\section{WILEY-VCH}

shanks (Figure 2C) and Utah-style pillars (Figure 2D)) for monitoring (and modulating) neural electrophysiology ${ }^{[4,40,41]}$. In vitro systems for studying the electrophysiology of organotypic tissues and dissociated neural cell cultures have also benefited from the microfabrication technology with the production of planar microelectrode arrays (MEAs) ${ }^{[2,25,28,42,43]}$. A major limitation of these in vitro and in vivo electrode arrays has been the difficulty in obtaining high enough signal-to-noise ratio (SNR) for resolving signals from neural cells. This difficulty arises partially due to high electrode impedance $\left(Z_{e}\right)$, resulting from reduced electrode surface area ${ }^{[27]}$. Additionally, scar tissue that forms after implantation in many cases can lead to significant loss of electrode performance due to distancing of neurons from the electrode surface and the scar tissue acting as an ionically-insulating layer (i.e., additional resistor in the electrical circuit between the neuron and the $\mathrm{R}_{\mathrm{e}}-\mathrm{C}_{\mathrm{e}}$ combination). Adverse tissue response can also result in complete device rejection within weeks after implantation, thus further decreasing the resolvable signal ${ }^{[44,45]}$.

Utilizing nanostructured material coatings on recording electrodes has become widely accepted as a method for decreasing impedance. Coating an electrode surface with a nanostructured material dramatically increases surface area and subsequently increases the electrode capacitance $\left(C_{e}\right)$ thus decreasing total electrode impedance ${ }^{[27]}$. Since the electrode material resistivity $\left(R_{e}\right)$ remains similar between conductive materials and the impedance is mainly due to the electrode-electrolyte interfaces (through electric double layer capacitance $\left(\mathrm{C}_{\mathrm{e}}\right)$ ), augmenting the area of this interface via nanostructuring is the preferred method for decreasing overall electrode impedance. Additionally, this increase in surface area is approximately inversely proportional to the feature size of the nanostructured material. This is due to the spherical electrode volume increasing as a function of the feature size to the third power $\left(\mathrm{r}^{3}\right)$ and the area increasing as a function of the feature size to the second power $\left(\mathrm{r}^{2}\right)$, resulting in an inverse (1/r) relationship between feature size and the effective surface area of the material. 


\section{WILEY-VCH}

One of the initial reports using this approach appeared in 1980 with the observation that electrochemical platinum deposition onto gold electrodes improved recording performance ${ }^{[46]}$. This process resulted in significant gains in SNR through a reduction in electrode impedance. The electrodeposited film growth results in a nanostructured platinum structure with feature sizes ranging from 10 s to 100 s of nanometers (commonly referred to as platinum black) on the surface of the electrode and has been shown to reduce impedance to a range of $1-100 \mathrm{k} \Omega$ at 1 $\mathrm{kHz}$ for electrodes in the $10 \mathrm{~s}-100 \mathrm{~s}$ of micrometer range (Figure 3A $)^{[46-51]}$. Although platinum black possesses the impressive electrochemical properties of platinum, it suffers from poor mechanical stability and is prone to delamination ${ }^{[51]}$. Therefore, more mechanically-stable metals have been investigated through nanostructuring the electrode surface. Some examples include iridium oxide ${ }^{[52,53]}$ and titanium nitride ${ }^{[53]}$ which can both be patterned with feature sizes ranging in 100 s of nanometers, as well as nanostructured gold ${ }^{[54-57]}$ and nanostructured platinum $^{[58]}$ which can be fabricated with smaller feature sizes in the 10 s of nanometer range. These materials have all been shown to reduce impedance to levels of $10 \mathrm{~s}$ to $100 \mathrm{~s}$ of $\mathrm{k} \Omega$ at 1 $\mathrm{kHz}$ for micro-scale electrodes capable of detecting single unit action potentials (Figure 3A $)^{[54-}$ ${ }^{60]}$. Since these materials are fabricated through controllable microfabrication techniques, their nanostructure can often be precisely tuned by optimizing the processing steps. The ability to tune nanostructure feature size is a major advantage over many of the electrochemicallydeposited films that are economical and easily accessible, yet traditionally suffer from poor reproducibility of film nanostructure.

Precise control of electrode nanostructure transcends the electrical improvements and creates unique opportunities to enhance the electrical interface. There has been significant research effort on utilizing nanostructured surfaces to influence cell behavior such as adhesion and motility ${ }^{[61-63]}$. Much of this effort has focused on utilizing nanostructure to control stem cell differentiation $^{[64,65]}$; however, the focus also encompasses the response of neural cells to 


\section{WILEY-VCH}

nanostructure to address challenges in neural recording. One of the largest hurdles facing the electrical monitoring of neural function via implanted electrodes is the foreign body response after implantation ${ }^{[44,45]}$. There are many in-depth review articles highlighting the cellular mechanisms influencing this response ${ }^{[66-68]}$. Briefly, trauma after implantation (both acute and chronic) causes a local inflammatory response, in which activated astrocytes gradually cover the electrode surface and deposit extracellular matrix to create a protective barrier between neurons and the electrode (Figure 4A) ${ }^{[68]}$. This scar tissue separates neurons from the electrode surface, reducing the recorded signal amplitude (Figure 4B) ${ }^{[44]}$. To this extent, some of the most promising results regarding nanostructured electrical interfaces have been utilizing nanostructure to directly control how neural cells adhere and spread over material surfaces. Neural cells (both neurons and glia) display a strong response to nanoscale feature sizes, such as surface roughness in the range of 30 to 100 nanometers $^{[69]}$, ridge widths in the regime of 500 to 2000 nanometers ${ }^{[70]}$, as well as surface morphology length scales ranging from 30 to 500 nanometers (Figure 3B) ${ }^{[54,71,72]}$. The underlying mechanisms of the cellular response to the nanostructure is complex and is a function of various mechanotransduction events ${ }^{[64]}$. One such mechanism is the regulation of focal adhesion formation on by the nanoscale spacing of biologically adhesive sites of the material (Figure 3B) ${ }^{[73-76]}$. Based on this principle, along with the notion that neurons and astrocytes form focal adhesion complexes influenced by different nanostructure size ranges ${ }^{[7]}$, it is possible to imagine the possibility of differentially controlling neural cell response by precisely tuning the underlying substrate nanostructure. Recently, we have demonstrated this phenomenon and its ability to not only improve SNR chronically, but also to increase the number of active electrodes over the entire duration of a mixed cortical cell culture $^{[54]}$. This nanostructure-mediated functionality taken together with the reduced electrode impedance stemming from electrode nanostructuring alone, is a promising demonstration of materials-enabled multifunctionality in a small footprint. Another promising avenue of mitigating adverse tissue response via material properties has been the use of compliant 


\section{WILEY-VCH}

materials as implant substrates to obtain a better mechanical matching between the interface and the neural tissue. Although this topic is beyond the scope of this review, multiple reviews have been recently published highlighting current efforts to utilize soft and stretchable materials for reducing chronic implant trauma caused by micro-motion of the electrodes, and for improving device interface through more conformal device geometries ${ }^{[24,78-80]}$.

In tandem, there have been considerable efforts towards the development of non-metallic materials for use as electrode coatings with a focus on conducting polymers and carbon-based materials $^{[81]}$. Conducting polymers, most notably poly(3,4 ethylenedioxythiophene) (PEDOT), have received significant attention due to their ability to form stable layers with small feature sizes of 10 s of nanometers through electrochemical deposition ${ }^{[82-85]}$. Similar to platinum black, the conducting polymer layer formed through this method is nanostructured and therefore benefits from a significant reduction in impedance to values between $1-10 \mathrm{k} \Omega$ at $1 \mathrm{kHz}$ for certain geometries (Figure 3A). Additionally, the polymer base of these materials makes an ideal scaffold for chemical functionalization and drug release from the polymer film ${ }^{[86-88]}$. This functionality will be covered in the Chemical Modulation section. On the carbon-based materials front, the main interest has been around either single-walled or multi-walled carbon nanotubes (CNTs) that are attached over a metal or semiconductor substrate and have typical feature sizes below 50 nanometers. Depending on how the CNTs are functionalized (vertically aligned or stacked), there can be a substantial increase in the electrochemically-accessible surface area, thus achieving low electrical impedances of 3 to $55 \mathrm{k} \Omega$ at $1 \mathrm{kHz}$ (Figure 3A) ${ }^{89-}$ ${ }^{93}$. However, the biocompatibility of CNT coatings is yet to be determined ${ }^{[94-96]}$. Recently, graphene, a two-dimensional carbon-based material, has received attention for use in electrical interfaces ${ }^{[97,98]}$. Although graphene is mostly planar and therefore cannot achieve the same surface area as CNTs, it has been shown to promote cellular adhesion ${ }^{[99,100]}$. This attribute is a potential avenue for enhancing SNR by obtaining a close physical coupling between the neuron 


\section{WILEY-VCH}

and the electrode (thus increasing seal resistance $\left(\mathrm{R}_{\text {seal }}\right)$ ). In addition, its embodiment as field effect transistors (FET) constitutes a new direction for high SNR neural recording seamlessly integrated with electronics ${ }^{[28]}$. The utilization of transistors as interfaces for electrical monitoring has seen a rise in popularity within the past few years. These technologies have successfully been fabricated using a range of the materials outlined above such as PEDOT and

graphene interfacing with gold or iridium ${ }^{[28,101-103]}$. Through a combination of optimal device geometries and material selection, FET have shown promise in amplifying the signal from the local field potentials via their modulation of gate dielectric polarization and consequently the channel conductance of the transistor.

Ultimately, utilizing nanostructured surfaces to improve the fidelity of electrically monitoring neural activity has led to important enhancements in our ability to acquire extracellular electrical information from neural systems. Nanostructure has played an instrumental role in enhancing recording fidelity by both decreasing electrode impedance $\left(Z_{\mathrm{e}}\right)$, providing cell type specific cues via nanostructure, as well as increasing the seal resistance $\left(R_{\text {seal }}\right)$ through promoting neuronal coupling to the electrode surface.

\subsection{Chemical Monitoring}

While neurons manifest their activity via both electrical (action potentials) and chemical (neurotransmitters) means, monitoring neural activity has traditionally been focused on electrophysiological recordings. In contrast to electrical communication that runs along the neuronal branches (i.e., axons, dendrites), chemical communication can influence a volume of disconnected neurons at once through the dispersion of neurotransmitters from the synaptic cleft to the extracellular space. This, in turn, leads to larger scale activation/inhibition of neural circuits. The most prominent chemical monitoring methods are electrochemical-based detection and microdialysis-based sampling (followed by detection through other modalities such as 


\section{WILEY-VCH}

fluorescence and electrical) ${ }^{[14]}$ (Figure 5). Briefly, electrochemical methods (voltammetry) offer rapid (sub-second) and direct detection of electroactive molecules ${ }^{[5]}$, such as dopamine and serotonin, but are prone to selectivity and biofouling issues. On the other hand, microdialysis-based sampling can monitor a wider range of molecules ranging from oxytocin (hormone important in bonding) to metabolites (such as glucose and lactate), but it has limited temporal resolution. In this section, we will focus on these two techniques, with a discussion of how micro- and nano-technology have improved the functionality of these chemical monitoring modalities.

\subsubsection{Electrochemical}

The operation principle of electrochemical monitoring is to apply a certain electrical potential (with respect to a reference electrode), which leads to the oxidation or reduction of a moleculeof-interest, yielding an electrical current, known as faradaic current (Figure 5A) ${ }^{[5]}$. The electrochemical potential at which this oxidation or reduction (redox) event occurs serves as a fingerprint for the molecule type. This method requires the molecules-of-interest to be electroactive ${ }^{[5]}$. Some examples of electroactive neuro-relevant molecules include biogenic amines (dopamine, norepinephrine, and serotonin) ${ }^{[5,104-106]}$, as well as their metabolites, glutamate, and glucose ${ }^{[107]}$. There are two dominant modes of this technique. The first one is constant-potential amperometry wherein, as the name entails, the working electrode is kept at a fixed potential and the number of electroactive species (whose oxidation potential lies below the applied potential) are oxidized or reduced when they reach the electrode. In this electrochemical protocol, there is no capacitive charging current (since the system is non-timevarying), where capacitive current typically disguises the faradaic current especially in working electrodes with large surface areas. Consequently, the number of molecules reduced or oxidized can simply be determined by $Q=z N F$, where $Q$ is the total electrical charge (produced due to a redox event), $z$ is the number of electrons transferred per ion, $N$ is the moles of molecules, 


\section{WILEY-VCH}

and $F$ is Faraday constant. However, the shortcoming with this method is that it lacks specificity, especially in vivo where there may be multiple electroactive molecules present. This method is therefore more suitable for conditions where the molecular content is well known. For example, in vitro cell culture, where the types of neurotransmitters present are well characterized, this method becomes powerful ${ }^{[104,108,109]}$. In this example, cells are cultured near a working electrode, where neurotransmitters released from vesicles at the axon leak into the extracellular space. While some neurotransmitters bind with the receptors on the dendrite to stimulate the next cell and some are oxidized at the electrode to yield neurotransmitter release information. The transient measurements provide release kinetics information with millisecond resolution, the time integral of these events yield the number of neurotransmitters released, and the shape of the spikes can be used for qualifying the neurotransmitter type and cell stimulationexocytosis delays ${ }^{[14]}$.

An alternative technique is fast-scan cyclic voltammetry, where the potential is varied rapidly $(100 \mathrm{~V} / \mathrm{s})$ across the range that covers the electroactive species. The advantage of this technique is that since a potential range is scanned, the different electroactive species can be identified by the potentials where a peak is observed. The challenge is that rapid cycling leads to large background currents due to capacitive charging, which reduces the signal-to-noise ratio (SNR) for the actual molecule detection. In order to mitigate this issue, several post-processing and other electrochemical techniques (e.g., square-wave or differential pulse voltammetry) have been devised ${ }^{[110]}$. The most popular neurotransmitter that is monitored with this technique has been dopamine, which plays a significant role in diseases such as Parkinson's and schizophrenia $^{[111,112]}$. Detection of norepinephrine and serotonin have been more challenging due to selectivity and electrode biofouling issues. Changing the sweep profile, as well as the range, can lead to oxidation of different species due to their different diffusion coefficients, multiple oxidation levels, and electron transfer rates. Using electrochemical scan waveform 


\section{WILEY-VCH}

modulations, a variety of different neurotransmitters have been detected, such as adenosine (which plays a role in regulating brain metabolism) in the presence of common interfering molecules such as hydrogen peroxide and $\mathrm{ATP}^{[113,114]}$ and methionine-enkephalin (opioid ${ }^{[115]}$ in the presence of catecholamines (e.g., norepinephrine).

The first electrodes used for electrochemical detection were carbon paste electrodes ${ }^{[16]}$ and metal wires. However, these systems lacked multiplexing capabilities and led to large tissue damage that likely resulted in artifacts in measurements. The onset of microfabrication techniques and miniaturization of electrodes generated exciting possibilities. Aside from being able to create arrays of electrodes that can monitor neurotransmitters in different anatomical regions with high spatial resolution due to smaller electrodes, the reduction of electrode size enhanced limits of detection (although with a downside of increasing settling/response time) ${ }^{[117-}$ 119]. One of the significant issues to consider when miniaturizing electrodes is whether the material of interest is microfabrication-compatible. For example, while carbon displays good biocompatibility and electrical properties, the conventional carbon paste electrode that has been the workhorse of electrochemistry could not easily be patterned via microfabrication techniques. An advancement over carbon paste has been the carbon fiber electrode ${ }^{[120,121]}$, which is typically sealed in a borosilicate glass capillary $(\sim 500 \mu \mathrm{m}$-diameter $)$ that is prone to breakage or in a more robust fused silica capillary $(\sim 100 \mu \mathrm{m}$-diameter $)$ that can reduce tissue damage during implantation ${ }^{[122]}$. More microfabrication-compatible materials such as gold and platinum have replaced carbon, particularly when photolithography is used for creating electrochemical arrays. Gold especially benefits from the well-established gold-thiol linker chemistry to further functionalize electrodes with capture/reporter moieties such as enzymes. However, carbon-based MEAs are still possible by creative techniques such as pyrolysis of organic material $^{[123]}$. 


\section{WILEY-VCH}

Emergence of nanotechnology, particularly the development of nanomaterials, allowed for the use of advanced coatings in electrochemical detection. In addition, nanofabrication allowed for reducing sensor footprint leading to better multiplexing. Nanostructure, has not only enhanced the limits of detection, but also has provided some solutions unique to the nanometers length scales $^{[124]}$. For example, Colinson et al., our group, and others have shown that nanoporous gold (np-Au) electrodes remain electrochemically active due to their intrinsic sieving function, where large proteins non-specifically adsorb onto the surface of electrode but not efficiently seal the pores, therefore small molecules (such as redox molecules) or fibrillar polymers (such as short nucleic acids) can permeate the porous electrode to sustain detection performance ${ }^{[125-}$ 127]. Similar to the carbon fiber electrode, carbon nanofibers have found use for both chemical and electrophysiological recordings ${ }^{[128]}$. Nanomaterials can also be seen as an add-on surface functionalization (described in Electrical Monitoring section), as a means to increase the effective surface area. For example, carbon nanotubes and graphene have been coated on various electrodes (e.g., carbon fiber, gold) to enhance limits of detection and selectivity ${ }^{[129]}-$ a good example is dopamine (Figure 6A and B). By leveraging the different charge transfer rates of ascorbic acid and dopamine, high selectivity measurements of dopamine in the presence of the interfering ascorbic acid has been achieved ${ }^{[130,131]}$. However, one challenge with increased effective surface area is the aforementioned high capacitive current (related to the electric double-layer capacitance, $\mathrm{C}_{\mathrm{e}}$, described in the context of recording electrodes) masking the faradaic current that is specific to the target molecule. An emerging field, nanofluidics, have shown promise in novel detection schemes, where electrochemical cycling of redox reaction in nano-cavities can enhance detection performance ${ }^{[132-134]}$.

The selectivity and specificity have been significantly improved by immobilizing enzymes specific to the neurotransmitter-of-interest. In general, the enzymatic action produces hydrogen peroxide, which is an electroactive species that can be easily detected by electrochemical 


\section{WILEY-VCH}

means. This approach also paves the road to detecting neurotransmitters that are not electroactive, such as glutamate ${ }^{[107,135]}$, acetylcholine ${ }^{[136]}$, and adenosine $e^{[137]}$ (Figure 6C). However, multiplexing different hydrogen peroxide-reporter enzyme systems is difficult, since it is not possible to distinguish between different enzymes that correspond to signal from different target molecules. Although this can be mitigated by immobilizing the enzymes on different electrodes in an electrode array to keep track of the enzyme activity at individual electrodes. In addition to enzymes, other capture moieties, such as aptamers, have shown promise in detecting psycho-stimulants, such as cocaine ${ }^{[138]}$.

\subsubsection{Microdialysis}

Microdialysis is based on collecting a liquid sample, typically through a semipermeable membrane (based on a concentration gradient across the membrane) coupled to a small capillary tube for further downstream analysis of the dialysate (Figure 5B $)^{[13]}$. By varying the size cutoff the semipermeable membrane, where nanoporous materials hold significant promise ${ }^{[139]}$, a variety of different molecule sizes can be detected, ranging from energetic molecules like glucose to larger proteins such as beta-amyloids ${ }^{[140,141]}$. However, in order to have enough SNR for reliable measurements, a large amount of sample (hence long collection duration) has traditionally been necessary. This, together with the latency resulting from molecules traversing the semi-permeable membrane driven by the molecular concentration gradient, adversely affects temporal resolution. Integrating this technique with capillary electrophoresis (that requires less sample volume) has improved detection limits considerably and hence reduced sampling times increasing temporal resolution ${ }^{[142]}$. Another challenge is the diffusion-related broadening of the sample fractions as they travel through the dialysis tube, called Taylor dispersion. This is an important obstacle to temporal resolution. Droplet-based approaches have largely mitigated this sampling/transport problem, where the samples are encapsulated in droplets, therefore there is no diffusion-broadening and the samples can be analyzed ex vivo, 


\section{WILEY-VCH}

which provides the opportunity to use a wide range of analytical techniques ${ }^{[142-144]}$ (Figure 6E). A device architecture, where micro-encapsulation is both used for delivering and sampling chemical moieties, has not been used in the context of neuroscience yet, but it has potential to be a useful tool to probe neural tissue (Figure 6F). Microfluidics, where channels with welldefined dimensions can be patterned on substrates via microfabrication-techniques, have drastically improved the microdialysis-based techniques ${ }^{[145]}$. This has become especially useful in on-chip generation of droplets for subsequent on-chip or off-chip detection.

In order to not deplete the fluidic environment of the sampled region (which could adversely affect physiological function), push-pull method of infusing physiological buffer via one capillary/microfluidic channel, while sampling with another has been employed (Figure 5B and 6 ${ }^{[142,146,147]}$. The probes used for microdialysis are usually a few millimeters long and a few hundreds of micrometers in diameter, while the capillary assemblies (such as for push-pull) are generally made by hand. Microfabrication technology for microfluidic channel definition permit more sophisticated designs without sacrificing precision. There is number of analysis methods used for characterizing the constituents of the dialysate. Traditionally, highperformance liquid chromatography (HPLC) with fluorescent or electrochemical reporters have been used, but now liquid chromatography coupled with mass spectroscopy is emerging ${ }^{[148]}$. As is the case for the majority of microfluidic devices, channel clogging is an obstacle to long term durability of microdialysis platforms. The semipermeable membrane is also prone to biofouling and clogging, yet nanostructured coatings have shown promise in alleviating some of these issues. To that end, several monolithic membranes have been devised including, deep-reactive ion-etched porous anodic alumina ${ }^{[139]}$ (Figure 6D) and nanoporous polymers obtained by laserinduced phase separation ${ }^{[149]}$. 


\section{WILEY-VCH}

Taken alongside electrophysiological recordings, the neurochemical information extracted from electrochemical and microdialysis approaches create a richer set of data to monitor neural activity. As in the case of electrical interfaces, microfabrication and nanostructured materials have not only improved the conventional techniques but also gave birth to unique capabilities.

\subsection{Optical Monitoring}

Optical monitoring techniques have become a promising modality for investigating neural populations in vivo, largely due to advances in microscope technology and the development of new fluorescent probes (Figure 7A). As photons are capable of traveling up to $600 \mu \mathrm{m}$ to $2 \mathrm{~mm}$ into neural tissue depending on the wavelength of light ${ }^{[3]}$, optical monitoring techniques are capable of observing large volumes of tissue with subcellular resolution, and without directly interacting with the tissue being interrogated. Additionally, recent advancements in chemistry and molecular biology have provided a suite of different fluorescent indicators that can monitor different aspects of neural signaling as well as label specific subsets of the neural population or subcellular domains. Apart from some newly developed label-free optical recording techniques such as stimulated Raman scattering microscopy ${ }^{[150]}$ and detection of fast intrinsic optical signals from membrane electromobility-induced cell deformation ${ }^{[151]}$, optical monitoring is fundamentally an indirect monitoring technique, as changes to membrane potential or ion flux must first be transduced by fluorescent indicators before they can be optically detected. Therefore, the fundamental parameters in determining the effectiveness of any optical recording modality (i.e., SNR and spatiotemporal resolution) are affected by both the recording device and the fluorescent indicator.

In optical monitoring techniques, the SNR is largely dominated by the efficiency and brightness of the fluorescent indicator. Unlike electrical recording modalities that monitor a large change in the voltage at the surface of the electrode, during optical measurements only a small number 


\section{WILEY-VCH}

of photons reach the detector, and if this number of photons become sufficiently small, the SNR will be negatively affected due to shot noise ${ }^{[152]}$. For this case, the SNR is now determined by both the change in fluorescence and the number of photons that reach the detector and is given by the following equation:

$\mathrm{SNR}=\frac{\Delta \mathrm{F}}{\mathrm{F}_{0}} \sqrt{\mathrm{n}}$

where $F_{0}$ is the baseline fluorescence, $\Delta F$ is the change in fluorescence and $\mathrm{n}$ is the number of photons that reach the detector ${ }^{[153]}$. To improve the SNR, two parameters can be altered, increasing the photon count or improving the relative fluorescence change $\left(\Delta F / F_{0}\right)$ of the fluorescent indicator. Increasing photon count can be accomplished in two relatively simple ways, either through increasing the number of fluorescent indicators in a given volume or by increasing the excitation intensity. However, neither of these options are ideal, as increasing the number of fluorescent indicators can lead to increased background fluorescence and metabolic load ${ }^{[153]}$, while increasing the excitation intensity can lead to phototoxicity and photobleaching ${ }^{[154]}$. Additionally, since the SNR is dependent on the square root of the photon count, it is typically considered more efficacious to increase SNR through improving the relative fluorescence change of the indicators $\left(\Delta \mathrm{F} / \mathrm{F}_{0}\right)$.

Using these optical monitoring techniques, it is possible to probe neural activity and the structure of the neural tissue. Derivatives of the original green fluorescent protein ${ }^{[155]}$ can be genetically expressed in different cell populations and can be used to monitor the structure of both neurons ${ }^{[156-158]}$ and glial cells ${ }^{[159,160]}$. Additionally, transgenic mice have been developed that express a wide variety of fluorescent protein emission wavelengths allowing for neighboring cells to be labeled with different colors, enabling the tracking of individual cells and synaptic connections over multiple time points ${ }^{[161-163]}$. An excellent example of the 


\section{WILEY-VCH}

capabilities of optical monitoring techniques is to track individual neuron and glial cells is given by the 'Brainbow' method, in which the Cre/lox recombination system is used to create cells that express a random combination of three to four different fluorescent proteins (XFPs) (Figure 7A). This random expression of XFPs leads to the labeling of individual cells with more than 90 discernible colors. Monitoring neural activity is typically achieved through genetically-encoded fluorescent indicators that monitor either membrane voltage or $\mathrm{Ca}^{2+}$ concentration (Figure 7B and C). Monitoring membrane voltage through genetically-encoded voltage indicators (GEVIs) is a more direct method of monitoring neural activity and is capable of detecting trains of action potentials at a rate of $200 \mathrm{~Hz}^{[164]}$; however, the low brightness of most GEVIs limit their in vivo application ${ }^{[165]}$. Tracking the changes in intracellular $\mathrm{Ca}^{2+}$ concentration, has been the gold standard for optical monitoring of neural activity, and has been used to investigate a wide variety of neural processes ${ }^{[166-168]}$. During action potentials, $\mathrm{Ca}^{2+}$ concentration can increase over 100-fold due to the opening of voltage gated calcium channels ${ }^{[169]}$, and the current generation of genetically-encoded calcium indicators (GECI) are capable of transducing individual action potentials and action potential trains ${ }^{[16,17,170]}$. GECI are typically preferred over GEVI because they provide increased SNR and increased brightness; however, it is important to note that increases in intracellular $\mathrm{Ca}^{2+}$ concentration does not necessarily indicate neural activity as $\mathrm{Ca}^{2+}$ is used for many other intracellular signaling pathways.

Improvements in microscopy technology for in vivo applications is a significant driving force for the use of optical techniques to monitor neural activity and has been covered in depth by a number of excellent reviews ${ }^{[3,20,21]}$. In short, the goal of all in vivo microscopy technologies is to increase the spatial and temporal resolution in addition to increasing recording depth. The simplest microscopy method is epifluorescence microscopy, in which the entire focal plane is visualized and a 3D reconstruction of the tissue volume can be formed by scanning along the 


\section{WILEY-VCH}

focal plane into the tissue depth ${ }^{[171]}$. Although this method has the lowest spatial resolution (largely due to decreased SNR stemming from background fluorescence), it has excellent temporal resolution as the entire $2 \mathrm{D}$ field of view is imaged simultaneously. It is therefore conducive to mapping neural connections across different brain regions ${ }^{[172]}$. To improve the spatial resolution, confocal laser scanning microscopy uses a pinhole to eliminate out-of-focus fluorescence and reduces background noise at the cost of temporal resolution (as the laser needs to be scanned across the $2 \mathrm{D}$ plane $)^{[173]}$. Two-photon and multi-photon microscopy improve recording fidelity by using femtosecond infrared laser pulses, which have higher penetration depths than visible light ${ }^{[174,175]}$. Additionally, the spatial resolution for these techniques is improved due to individual photons not having enough energy to excite a fluorescent indicator. The fluorescence therefore is tightly localized at the focal point, where there is enough photon density for individual fluorescent indicators to absorb multiple photons. As with confocal laser scanning, two-photon microscopy requires the infrared laser to be scanned across the sample volume, and thus the improved recording depth and spatial resolution comes at the cost of temporal resolution. To improve the temporal resolution of two-photon and multi-photon microscopy techniques, a number of approaches have been developed to multiplex the number of scanning points including temporal multiplexing ${ }^{[176]}$, wavelength multiplexing ${ }^{[177]}$, and holographic multiplane strategies ${ }^{[178]}$.

\subsubsection{Cortical Imaging}

As most of the optics and actuators are located in the microscope assembly, the actual neural interface is typically only used to relay optical information. Chronic optical windows are the simplest form of optical neural interface and are comprised of a fixed glass slide placed over a craniotomy through which optical microscopy images can be recorded ${ }^{[179-182]}$. Optical windows are less invasive than other neural interfaces for optical recording as the glass slide does not 


\section{WILEY-VCH}

penetrate the neural tissue. Furthermore by using two-photon microscopy, it is possible to image through a thinned portion of the skull eliminating any contact between the brain and interface $^{[183]}$. However, even when using multi-photon microscopy, single cell resolution can only be maintained to a depth of $\sim 800 \mu \mathrm{m}$ into the neural tissue ${ }^{[179-181]}$; therefore, chronic optical windows are limited to recording from the surface layer of the cortex. In order to visualize neurons deeper within the cortex and at different angles, right angle prisms have been implanted into the cortex of mice that provided the capability to observe entire cortical columns ${ }^{[184,185]}$; however, due to the size and geometry of the prisms, they are still limited to cortical imaging.

\subsubsection{Deep Brain Imaging}

Beyond $\sim 800 \mu \mathrm{m}$, photon scattering becomes prohibitive to imaging at a single cell resolution, and therefore implanted neural interfaces are required to reduce the distance that the photons need to travel through the tissue (Figure 8). Optical monitoring of deep brain structures is typically accomplished through the insertion of microscale optical fibers into the neural tissue. The two most common optical fibers used are gradient-index (GRIN) fibers and fiber bundles. GRIN fibers provide the best spatial resolution when monitoring deep brain structures, as the GRIN fiber acts as a micro lens that can refocus a laser focal point scanned on the proximal end of the GRIN fiber into the neural tissue ${ }^{[186]}$. GRIN fibers are compatible with most microscopy techniques and have been used with epifluorescence, confocal and two-photon techniques ${ }^{[187-}$ ${ }^{190]}$. However, GRIN fiber diameter is relatively large (ranging from $350 \mu \mathrm{m}$ to $1000 \mu \mathrm{m}$ ) and they are not flexible ${ }^{[191]}$. An attractive alternative is fiber bundles consisting of thousands of closely packed step-index fibers that can be used instead of GRIN fibers to impart flexibility and enhance miniaturization ${ }^{[191]}$. The main drawback of fiber bundles is that the individual fibers can only relay light intensity to and from the target tissue, and thus the image is formed by combining the fluorescent intensity from each fiber with the reconstruction of each fiber's placement within the fiber bundle. Therefore, the spatial resolution is limited by the individual 


\section{WILEY-VCH}

fiber diameter and spacing. For both GRIN fibers and fiber bundles, the microscopy setup is located at the proximal end of the fiber. In the case of confocal and two-photon microscopy, the laser is scanned at the proximal end of the fiber and relayed to the neural tissue. An alternative to optical fibers is the direct implantation of microfabricated complementary metaloxide-semiconductor (CMOS) image sensors ${ }^{[192]}$. These sensors eliminate the need to insert a relatively blunt optical fiber into the neural tissue, and they can be arranged in a similar style to the Michigan array to decrease the total implanted footprint. However, CMOS image sensors still suffer from poor spatial resolution due to the resolution being determined by the size and arrangement of the individual sensors.

Overall, optical monitoring techniques can provide unique information from the other recording modalities, most notably the ability to monitor changes to the structural architecture of both neurons and glial cells. Additionally, as photons can travel through tissue, optical techniques are able to monitor neurons from a greater distance (Figure 8), and the formation of scar tissue is less impactful to their functionality. However, ultimately the exact number of fluorescent indicators located within a cell is typically not known, and therefore most optical monitoring techniques are only suitable for qualitative assessments of neural structure and activation.

\subsection{Summary of Interfaces for Monitoring Neural Activity}

Overall, electrical interfaces have been the most popular tools for monitoring neural activity. This is in part due to the ease of recording by interfacing neurons to an electrode and relative practically in multiplexing the number of electrodes (compared to chemical and optical devices). Direct electrochemical monitoring (without functionalization with intermediate molecules such as enzymes) of neurotransmitters and metabolites offer similar advantages, yet multiplexing these chemical interfaces have not been trivial. Microdialysis-based interfaces are more complicated and more challenging to multiplex; however, they offer real-time monitoring 


\section{WILEY-VCH}

of multiple analytes with high specificity. Optical interfaces generally require reporter molecules to transduce the signals, which makes them more complicated than electrical and chemical interfaces, unless well-defined reporters (such as for calcium signaling) are employed. However, optical interfaces allow for monitoring large numbers of neurons at once and may be more conducive to in vivo studies. Overall, each modality captures a distinct portion of neural activity, which underlines the importance of multifunctional interfaces in obtaining a more complete picture of neural circuit operation.

\section{Modulating Neural Activity}

\subsection{Electrical Modulation}

In addition to monitoring neural activity, electrical interfaces are widely used as a method to modulate electrophysiological activity by stimulating the neural tissue. As depicted in Figure 1, the electrical interface can be represented as electrical coupling of a neural cell to an electrode. Although the electrical parameters defined in the context of neuron-electrode equivalent circuit (Figure 1) remain important, other critical parameters emerge for the case of electrical modulation. These parameters are the mechanism of charge injection (e.g., capacitive or faradaic) and the charge injection capacity (CIC), which is defined as the amount of charge that can be injected at a given current or potential before inducing irreversible chemical reactions at the electrode surface (Figure 9). Both of these mechanisms are dictated by the material properties and effective surface area of the electrode. There are multiple reviews on the mechanisms of charge injection and their impact on neural tissue ${ }^{[4,193]}$. In a purely capacitive process, charge is transferred via electrically-induced changes in the electrical double-layer at the electrode-neuron interface, without the generation or consumption of chemical species. Conversely, in the faradaic process, the electrode participates directly in a redox reaction that produces or consumes charged chemical species. This section outlines how nanostructured 


\section{WILEY-VCH}

materials have been used to improve electrical modulation of neural tissue through altering the properties of the electrical modulating interface.

Similar to the monitoring of neural function, implanted devices for the electrical modulation of neural tissue started being investigated in the 1950s and 60s primarily as implantable wire electrodes (Figure 2A) ${ }^{[37,38]}$. These wire implants facilitated the modulation of large tissue regions, but given their 100 s of micrometer scale it proved difficult to selectively modulate a desired tissue region. The first attempts to improve spatial resolution were through the implementation of microwire arrays. These arrays consisted of tens of insulated gold wires with exposed platinized tips ${ }^{[194]}$. As a result of the advances in silicon-based microfabrication, penetrating implants with micrometer-sized electrode arrays such as the Michigan and Utah arrays (Figure 2C and D) have enabled significantly improved selectivity in the modulation of the neural tissue. However, as electrode size is reduced to the micrometer scale, the charge density at the electrode must be increased to compensate for the reduced surface area. One method of achieving higher charge density is simply by increasing the charge being injected through the electrode itself. This makes the selection of material charge injection mechanism extremely important to avoid electrode breakdown or tissue damage due to production of reactive ion species beyond a certain stimulating current.

The limitation imposed by the charge injection mechanism makes certain materials, such as gold, less attractive as a stimulating electrode due to their propensity to ionize permanently into the solution through faradaic charge injection. Since capacitive charge injection is ultimately the safest method to inject current into neural tissue, due to the absence of any chemical reactions, ceramics such as titanium nitride as well as carbon-based electrodes that act entirely through capacitive injection have seen extensive use ${ }^{[4]}$. However, since faradaic processes can achieve significantly higher amounts of charge transfer, materials such as platinum ${ }^{[47,48,50,58]}$, 


\section{WILEY-VCH}

platinum iridium ${ }^{[4]}$, and iridium oxide ${ }^{[48,53,195]}$ which inject charge through pseudo-capacitive mechanisms, are some of the most popular material options. Additionally, the conducting polymer PEDOT has received attention for stimulation despite it acting primarily through faradaic mechanisms. This is primarily due to the reported electrochemical stability of the PEDOT films and their diffusion-limited transfer of ions away from the films enabling the rebinding of ions inside the polymer without interaction with the external tissue ${ }^{[81,82,86]}$.

The other mechanism through which charge injection can be improved is by increasing electrode CIC. Nanostructuring of electrode surfaces can significantly improve CIC through increases in effective surface area. The most commonly used nanostructured surface remains electrochemically deposited platinum (platinum black). Platinum black-coated electrodes typically have a CIC in a range of $0.3-0.4 \mathrm{mC} / \mathrm{cm}^{2}$, which is approximately 50 to $100 \%$ larger than the CIC of $0.2 \mathrm{mC} / \mathrm{cm}^{2}$ for planar platinum (Figure 10) ${ }^{[48,50,58]}$. Although the CIC is still low, the ease of platinization makes platinum black an attractive method for coating electrode surfaces. Another frequently used material, titanium nitride, which acts through purely capacitive charge injection, has been shown to achieve a CIC of $0.87 \mathrm{mC} / \mathrm{cm}^{2}$ through nanostructuring (Figure 10 ${ }^{[53,196,197]}$. Further improvements in purely capacitive-based CIC have been observed through utilizing carbon nanotube (CNTs) based electrodes. These surfaces take advantage of the large electrochemical surface area of CNTs to achieve increased CICs. In one example, Wang et al. demonstrated a CIC of $1.6 \mathrm{mC} / \mathrm{cm}^{2}$, approximately four times that of platinum black electrodes (Figure 10 ${ }^{[91-93,198]}$. However, even better electrode performance can be observed through the nanostructuring of iridium oxide by altering fabrication parameters to create nanoscale roughness on the surface. These nanostructural changes have been shown to result in large increases in CIC to over $4 \mathrm{mC} / \mathrm{cm}^{2}$ (Figure 10 ${ }^{[48,53,195]}$. Finally, the conducting polymer PEDOT has gained popularity due to its efficaciousness in both monitoring and modulating neural activity ${ }^{[81,86]}$. With a reported maximum CIC of $2.3 \mathrm{mC} / \mathrm{cm}^{2}$, it is on par with 


\section{WILEY-VCH}

CNT and titanium nitride functionalized electrodes and offers additional options for the further functionalization of the device surface to give multifunctionality ${ }^{[199]}$. Another approach to preserve the multifunctionality of other nanostructured materials is the templating of stimulating electrode materials over nanostructured materials. To that end, iridium oxide has been conformally electro-deposited on nanoporous gold (np-Au), resulting in a nanostructured iridium oxide surface ${ }^{[200]}$.

As the predominantly utilized method of neural modulation, identifying new methods to integrate electrical modulation functionality into existing devices is an important step towards building multifunctional interfaces. Utilizing nanostructured materials as electrode coatings to attain high charge injection capacities, it has become possible to inject biologically-relevant charge densities from micropatterned electrodes with a small footprint while minimizing irreversible chemical reactions.

\subsection{Chemical Modulation}

Although electrical stimulation has been the most commonly used technique for modulating neural activity and optogenetic stimulation has emerged as a tool with previously unimaginable capabilities, direct chemical modulation of the neural activity, for example via controlled delivery of soluble neuromodulators remains a powerful tool for stimulating the neural tissue. To that end, there have been numerous techniques, where external stimuli, such as acoustic or magnetic energy, burst drug carrying micelles delivered through blood infusion or microinjection into the brain ${ }^{[201]}$. Chemical modulation through soluble factor delivery has been used extensively in the context of reducing astrogliosis, including therapeutics for alleviating foreign body response, such as anti-inflammatory agents (e.g., dexamethasone, $\alpha$-melanocyte stimulating hormone $(\alpha \mathrm{MSH}))^{[202-206]}$, neurotrophic factors (e.g., nerve growth factor (NGF), neurotrophin-3 (NT-3), and brain-derived neurotrophic factor (BNDF)), other growth factors 


\section{WILEY-VCH}

(e.g., ciliary neurotrophic factor (CNTF), fibroblast growth factors (acidic and basic, aFGF and bFGF), transforming growth factor- $\beta$ (TGF- $\beta$ ), and glial derived neurotrophic factor (GDNF)) [207,208]. Other less common pharmaceutical approaches include anti-TGF, interleukin-10, and even antibodies against astroglial markers ${ }^{[209]}$. Immobilized chemical moieties on surfaces are useful in modulating cellular behavior, by either influencing physical properties (e.g., hydrophilicity) or direct biochemical activity of the electrodes (via immobilized growth factors and/or adhesion biomolecules). Particularly this has found use in reducing immune-reactivity of electrodes by surface treatment approaches, particularly the surface-immobilization of biomolecules have shown promise in promoting neural growth and attachment ${ }^{[210]}$. These molecules include common extracellular matrix (ECM) elements (e.g., laminin ${ }^{[211]}$ and poly-Dlysine ${ }^{[44]}$ ) and surface recognition molecules (e.g., N-cadherin, NCAM, L1, CHL1) ${ }^{[209,212]}$. Recently, shorter peptides that are reminiscent of larger ECM molecules have not only shown to improve neuronal survival, but also hint at the possibility of cell type-specific adhesion ${ }^{[211-}$ ${ }^{213]}$. In this section, we focus on approaches for delivering soluble chemical factors to neural tissue with a particular emphasis on modulating neural behavior (Figure 11).

\subsubsection{Fluidics-Based Delivery Approaches}

Conventionally, brain infusion has been accomplished via cannulas made of materials such as steel and glass. Typically, the chemical factor containing fluids are microinjected through this cannula via tubing attached to a syringe pump. This has been successful in the delivery of therapeutics and transfection agents for gene therapy and optogenetics. For example, a neurotrophic factor has been successfully infused into the brain via catheters for management of Parkinson disease ${ }^{[214]}$. However, this technique lacks spatial resolution, especially in probing small circuits in the brain. In addition, the insertion of cannula/catheters results in significant tissue damage and these systems are not compatible with studies involving moving animals. There have been approaches to incorporate an electrode in this system such as carbon fiber or 


\section{WILEY-VCH}

tungsten wire for recording while injecting pharmaceuticals. These electrodes have also been used for keeping track of the number of molecules delivered by electrochemically oxidizing a reference molecule mixed in with the active molecules as they are leaving the capillary ${ }^{[215]}$.

Introduction of micropatterning techniques led to integration of microfluidic channels into electrical probes and allowed for precise infusion of drug molecules into neural tissue. A recent review by Sim et al. overviews fabrication techniques, materials, and potential applications of microfluidic-based neural probes ${ }^{[216]}$. An example is a shank electrode with buried microfluidic channels, where the electrode tips are coated with platinum ${ }^{[217]}$. The microfluidic channels can be connected to the surface of the probe using etched through-holes for drug delivery and simultaneous recording (Figure 12A). Devices that enable multiplexed delivery of different pharmaceuticals have been demonstrated ${ }^{[218-220]}$. Microfluidic probes, similar to other probes, have been made of hard materials, such as silicon (better studied microfabrication processes for patterning), and soft materials (e.g., SU8 ${ }^{[221]}$, polyimide ${ }^{[222]}$, parylene- $\mathrm{C}^{[223]}$ ) to reduce micromotion-related adverse tissue response. For miniaturized systems to be used in moving animals, it is essential that the dependence on external components are minimized, therefore use of syringe pumps or large external reservoirs is not practical. To that end, there have been developments on micropumps ${ }^{[224]}$. For mechanical pumps, typically there is a diaphragm that pushes the liquid out, where the diaphragm can be actuated via thermal, electrostatic, electromagnetic, and electrochemical modes. The control of volume and rate of flow, power

consumption, and non-hazardous actuation are key requirements for all pumps ${ }^{[225]}$. Another important aspect is fluid storage. In addition to there being limited space to include a small reservoir, it is also important to ensure the reservoir can safely contain the liquids. Several microfabrication strategies have been employed to address this issue, such as check valves, hermetic sealing, and materials to prevent fluid evaporation ${ }^{[226-228]}$. 


\section{WILEY-VCH}

\subsubsection{Coating-Based Delivery Approaches}

A significant impact/contribution of nanotechnology has been on development of nanoparticlebased theranostic modalities, where the influence on monitoring can be seen in optical monitoring of brain activity. On the therapeutic front, nanoparticles loaded with drug molecules have been used for delivering their cargo upon various triggers (optical, magnetic, acoustic, thermal). An excellent set of reviews covering nanoparticle-based delivery methods can be seen elsewhere ${ }^{[229-233]}$. In this review, the focus is on nanostructured coating-based modalities. Nanomaterials with their high effective surface area for increased loading capacity and tunable morphology have provided new avenues for controlled drug delivery to the neural tissue. For these types of coatings, where the surface area-to-volume ratio is high, the majority of the transport is due to the surface-molecule interactions, which not only results in high loading capacity but also enables the ability to control release kinetics via modulating the surfacemolecule interactions by a variety of modalities (e.g., electrical to switch electrode surface charge). Our group has demonstrated that nanoporous gold (np-Au) coatings can retain physiologically-relevant doses of anti-mitotic small molecules drugs (Ara-C) to suppress astrocytic proliferation in an in vitro astrocyte culture model ${ }^{[234]}$. This molecular release can be gated by changing the type and strength of ionic species ${ }^{[235]}$. The np-Au coatings can also be used for capacitive loading and release of ionic small molecule drugs via iontophoresis ${ }^{[236]}$. Abidian et al. used PEDOT nanotubes for electrical release of dexamethasone by varying electrochemical potentials $^{[237,238]}$ (Figure 12C). Layer-by-layer deposition is another attractive technique that provides precise control of layer thickness of various materials including, carbon nanotubes $^{[239]}$. There are examples of drug-eluting coatings using polymeric coatings on the electrodes $^{[203]}$, as well as carbon nanotube-based coatings for release of dexamethasone to reduce gliosis ${ }^{[204]}$. Unfortunately, nanomaterial-based coatings are not attractive options if they cannot be successfully integrated into electrodes with well-defined techniques such as 


\section{WILEY-VCH}

microfabrication processes. To that end, electrochemical methods, although reproducibility may be an issue, can easily be integrated into electrodes where existing electrodes are used as working electrodes for nanostructured material growth. Other desirable processes commonly used are physical vapor deposition techniques (such as evaporation and sputtering). A universal challenge to nanostructured coating-based drug delivery schemes is to reduce molecular release during the OFF state. Charged polymer-based structures have reduced the undesirable release by strong electrostatic containment of drug molecules on the polymers. Others, such as Cui et al., have used polymers (polypyrrole) to cap carbon nanotubes to reduce molecular release of dexamethasone via steric hindrance ${ }^{[204]}$.

Taken together, the miniaturization technology, coupled with the interfacial phenomena emerging at the nano-scale materials and channels, have paved the way to miniaturizing neuromodulator delivery schemes and enhanced the possibility to integrate them into multifunctional neural interfaces for monitoring and modulation of neural activity.

\subsection{Optical Modulation}

Advances in optogenetic technologies have elevated the interest in optical techniques to modulate neural activity. As optogenetics has become a powerful tool for neuroscientists, there have been a number of excellent reviews published ${ }^{[15,22,23,165]}$. Briefly, optical modulation of neural activity is dictated by the genetic expression of light-activated transmembrane ion channels (opsins) in targeted neural populations ${ }^{[240]}$. There are now many different opsins to both activate and inhibit neural activity that are excited by many different wavelengths of light ${ }^{[241-245]}$. Additionally, expression of these light-activated ion channels can be targeted to specific neural populations, thereby allowing the optical stimuli to selectively modulate the neural activity of a specific sub-population of genetically defined neurons even when a large volume of tissue is exposed to the optical stimulus ${ }^{[240]}$. This has allowed researchers to increase 


\section{WILEY-VCH}

the understanding of functional neural anatomy through improved brain mapping ${ }^{[241,246]}$ and investigate the roles that specific neural circuits may play in behavior and disease ${ }^{[1,247,248]}$.

\subsubsection{Photon Delivery}

The role of neural interfaces during optical modulation techniques is to deliver light to the neuron population of interest at the minimum required irradiance $\left(\sim 1 \mathrm{~mW} / \mathrm{mm}^{2}\right.$ for optogenetic activation $^{[240]}$ ) for optical modulation. As most light-activated ion channels used in optogenetic controls respond most strongly to light in the visible spectrum, scattering effects through the neural tissue become increasingly significant and substantial attenuation of the optical stimuli occurs within a few hundreds of micrometers ${ }^{[40]}$. Longer wavelength, two-photon stimulation is possible with optogenetics to increase the penetration depth of the light source and to achieve sub-cellular spatial resolution of the optical stimuli ${ }^{[249-252]}$. However the single channel conductance of individual opsins are generally low and typically have fast kinetics meaning that multiple opsins in an area much larger than the typical two-photon excitation volume need to be stimulated nearly simultaneously to induce an action potential ${ }^{[253]}$. While the increased spatial resolution from two-photon optical stimulation allows for the modulation of subcellular compartments of neurons (e.g., individual dendrites) ${ }^{[249,250]}$, it also requires complex scanning and stimulation methods to activate the neuron. Therefore, as optogenetics is already capable of selectively activating genetically defined sub-population of neurons, most optical modulation approaches involve the use of visible light delivered to relatively large volumes of neural tissue $^{[40]}$.

The most common light delivery interface used during optogenetic studies are commercially available optical fibers coupled to external light sources, often with sharpened tips to reduce tissue damage during implantation ${ }^{[240,254-256]}$ (Figure 13A). This approach is commonplace as optical fibers are relatively inexpensive, simple to fabricate and assemble, and can typically 


\section{WILEY-VCH}

illuminate entire brain regions in small animal models ${ }^{[257]}$. However, optical fibers have several drawbacks, as they are fragile, restrict movement, only allow for optical stimulation at a single site, and are not compatible with traditional microfabrication techniques. An inexpensive alternative is the use of chronic optical windows in conjunction with head-mounted light emitting diodes (LEDs) that allow free movement, and are significantly less expensive than optical fibers $^{[258,259]}$. Just as with optical modulation, chronic optical windows are typically considered less invasive as the interface is not penetrating the brain, but also are only capable of modulating neurons in the cortex. Chronic optical windows are compatible with transparent micro-electocotography $(\mu \mathrm{ECoG})$ arrays fabricated from materials such as parylene-C, graphene and ITO, which can obtain ECoG recordings from neurons directly beneath the recording sites ${ }^{[97,260,261]}$ (Figure 13B). Microscale waveguides have gained increased popularity as they can be fabricated using standard microfabrication techniques and can be used to optically modulate multiple regions of the brain at any depth. The waveguides are typically coupled to an external light source and fabricated using an oxynitride ${ }^{[262,263]}$ or SU8 core ${ }^{[264,265]}$ (Figure 13C). Since they are fabricated using traditional microfabrication techniques, the waveguides can be multiplexed into Michigan-style ${ }^{[262,264]}$ and Utah-style ${ }^{[266]}$ probe configurations. Furthermore, flexible waveguides have been fabricated using two types of PDMS to produce a more "biocompatible" interface ${ }^{[267]}$.

An alternative to delivering optical stimulation from an external light source is the direct implantation of $\mu \mathrm{LED}$ arrays, which are fabricated using gallium nitride $(\mathrm{GaN})$ on a sapphire substrate using standard microfabrication techniques ${ }^{[268]}$ (Figure 13D). These $\mu$ LEDs and array patterns can be fabricated in a variety of geometries to improve the spatial resolution of the stimulating light, and the arrays can be designed to modulate a large volume of tissue while displacing the smallest volume possible. Additionally, it is now possible to transfer $\mu$ LED arrays onto flexible, polymeric substrates to reduce the mechanical mismatch and chronic 


\section{WILEY-VCH}

immune response ${ }^{[269-271]}$. However, because LEDs emit non-coherent light, the effective penetration depth of the optical stimuli is reduced and therefore the LEDs need to be in closer proximity to the target cells. Additionally, thermal management of the device can become an issue, especially when large LEDs or closely spaced LEDs arrays are used ${ }^{[272-274]}$. Furthermore the thermal conductivity of the substrate can greatly impact the thermal fluctuations around the $\mu \mathrm{LED}$, with flexible, polymeric substrates concentrating thermal fluctuations due to their lower thermal conductivity ${ }^{[274]}$.

\subsubsection{Optical Nanomaterials}

Nanoparticles can be used to optically modulate neural activity in conjunction with or independently from optogenetic techniques. Upconverting nanoparticles act in a similar fashion to two-photon microscopy-compatible fluorescent indicators, in which they absorb multiple photons of infrared wavelength light and emit shorter wavelength light ${ }^{[275-278]}$. As many popular opsins require visible light for activation, these upconverting nanoparticles can improve the penetration depth of optical stimuli ${ }^{[275-278]}$. Alternatively, thin-films formed from $\mathrm{HgTe}$ nanoparticles have been shown to directly convert optical stimulation to electrical stimulation, and could possibly induce optically-modulated neural activity if embedded within the cellular membrane ${ }^{[279]}$. However there are a few barriers to the successful implementation of $\mathrm{HgTe}$ nanoparticles to induce optically-controlled neural modulation, most notably the cytotoxicity of the HgTe nanoparticles and the difficulty of localizing them to the cellular membrane ${ }^{[23]}$. Alternatively, gold nanoparticles can induce optically-controlled neural modulation through the conversion of an optical stimuli to heat through surface plasmon resonance ${ }^{[280-282]}$. Depending on the optical stimuli, gold nanoparticles can inhibit neural activity during sustained optical illumination through the activation of temperature sensitive potassium ion channels ${ }^{[281]}$, or can activate neural activity during pulsed optical stimulation through temperature induced changes 


\section{WILEY-VCH}

to cellular membrane capacitance ${ }^{[280,282]}$. Nevertheless, while gold nanoparticles are considered more biocompatible than HgTe nanoparticles, the cytotoxicity of gold nanoparticles is still debated $^{[283-285]}$ and localizing them to the appropriate cell membrane in vivo is still considered non-trivial ${ }^{[2]}$.

As optogenetics technology became more mature, optical modulation techniques have become the premiere tool to interrogate neural circuits for fundamental neuroscience research. Optical modulation is unique in that it is capable of modulating specific subsets of neural populations and even subcellular domains of individual neurons. Furthermore, similar to optical monitoring, as photons are able to travel through tissue, optical interfaces are less affected by the chronic immune response and glial scar formation. However, the use of optical modulation techniques requires either the genetic modulation of the target neuron or the use of potentially cytotoxic nanoparticles that may impact its translation to the clinical setting.

\subsection{Summary of Interfaces for Modulating Neural Activity}

Each interface modality discussed in this section offer unique opportunities to modulate the behavior of neural tissue. Similar to the interfaces used for monitoring neural activity, the electrical interface is the most commonly used methods for modulation; however, while this method is easy to combine with existing recording electrode architectures, it has very low spatial and cellular specificity. In addition, it can only be used to trigger neuronal firing. Optical interfaces coupled with optogenetic techniques largely mitigates the shortcoming of electrical interfaces, as they are able to turn on and off specific neuronal cell types, creating an extremely powerful tool for neuroscience. However, the requirement to use viral vectors to transform the neuronal cells raises issues in its translation to clinical use. Interfaces for chemical modulation, especially in situ delivery of neuromodulators and therapeutics, holds promise for management 


\section{WILEY-VCH}

of complex neurological disorders, such as epilepsy. Advancements in optical and chemical modulation modalities are expected to surpass the capabilities of electrical interfaces if their ease of use and fabrication can meet that of electrical interfaces.

\section{Combinatorial Material Libraries}

A major limitation to determining the correct material attributes for multifunctional interfaces and devices is the enormous effort and time required to test combinations of different attributes. Therefore, testing each single combination of material attributes (e.g., morphology and electrical properties) and establishing relevant controls within the same experiments becomes prohibitively cumbersome and costly. In order to mitigate this issue, there has been a push towards high-throughput testing platforms or 'combinatorial material libraries' that can be used to rapidly identify the optimal material attributes for a specific application ${ }^{[286-288]}$.

Many implementations of combinatorial material libraries have focused on soft biomaterials with a focus on regenerative medicine. These libraries have been utilized for characterization of hydrogels ${ }^{[289]}$, polymers ${ }^{[290]}$, as well as extracellular matrix components ${ }^{[291]}$ both in vitro $^{[292,293]}$ and in vivo ${ }^{[291]}$ (Figure 14). Recently, Beachley et al. demonstrated the patterning and fabrication of both $2 \mathrm{D}$ and $3 \mathrm{D}$ tissue arrays as a high-throughput method to study tissuespecific cellular responses ${ }^{[293]}$ (Figure 14C). Using the 2D implementation of a tissue-array, they investigated the response of both stem cells and melanoma explants to bone, brain, cartilage, collagen, lung, and spleen tissues in parallel. The resulting cultures were then studied at a proteomic level to provide insight into how these cells differ in their interactions between the different tissues. Similarly, Hook et al. have demonstrated two realizations of combinatorial material libraries for the investigation of polymers that reduce bacterial adhesion ${ }^{[290]}$, as well as in vitro biomaterial development ${ }^{[287]}$ (Figure 14A and B). Surprisingly, the application of 


\section{WILEY-VCH}

combinatorial material libraries to neural interfaces have been limited. Some examples include using ECM protein combination to identify compositions and geometries that lead to desired cell phenotype (i.e., neuron or astrocyte) upon insoluble factor-induced differentiation of neural stem cells ${ }^{[294]}$.

Although many of these material libraries consist primarily of soft biomaterials (e.g., hydrogels, tissue, etc.), there have been advancements in fabrication of combinatorial libraries of nanostructured materials ${ }^{[286,295]}$. Recently our group has demonstrated a method to create electrode morphology libraries of nanoporous gold (np-Au) to rapidly screen for feature sizedependent processes. These material libraries were photolithographically patterned and subsequently annealed via laser irradiation at different energies to coarsen each np-Au morphology to display a prescribed median feature size ranging from 30 to over 400 nanometers $^{[295]}$ (Figure 14D). In a recent article, we identified a regime of nanoporous gold feature sizes that selectively reduced the spreading of astrocytes while not affecting neuronal coverage. This selective coupling along with the reduction in impedance from nanostructure (compared to planar gold) improved SNR by approximately $50 \%$ above the improvements already seen over standard $\mathrm{np}-\mathrm{Au}^{[54]}$. Additionally, we have also employed our material libraries to study how far-field fluorescence from cell-bound fluorophores changes as a function of feature size. In this work, we demonstrated that even with identical base material (e.g., gold) the scattering due to nanostructure can significantly decrease perceived fluorescence intensity from a planar counterpart ${ }^{[296]}$ (Figure 14E and F). This study emphasized that quantitative immune-fluorescence should be used with caution when evaluation the influence of nanostructured materials on cell behavior, such as in the case of astrogliotic response to nanostructured electrodes. 


\section{WILEY-VCH}

These recent reports highlight the benefit of studying processes as a function of material nanostructure as well as how utilizing higher throughput methodologies such as combinatorial material libraries facilitate this investigation. The translation of this approach towards studying other processes such as drug release, material functionalization, optical properties, and biocompatibility will undoubtedly lead to enhanced discovery of regimes of feature size that result in enhanced performance for neural interfaces.

\section{System Integration, Closed-Loop Control, and Multifunctionality}

Multifunctionality, which we have previously described as the ability to both monitor and modulate neural activity, is a desirable trait for neural implants in both the clinical and research setting. In the research setting, it is possible to treat a neural circuit as a system to be characterized via systems identification principles, that is, to systematically modulate the system and monitor the outcome to develop transfer functions that describe the system behavior. In the clinical setting, multifunctional devices promise the ability to monitor the neural tissue for pathological signals and respond with an appropriate pharmaceutical or therapy in real time $^{[297]}$. The various modalities described above (electrical, chemical and optical) allow for modulation and monitoring neural systems within a rich parameter space, with each modality providing certain advantages and limitations. A major remaining challenge is to integrate these modalities within a small form factor to ensure functionality without sacrificing spatial or temporal resolution and to minimize the chronic immune response to the implant. Here, we provide an overview of some of the major considerations when designing multifunctional neural interfaces, along with some examples of successful approaches towards this goal.

As can be seen in Figure 15, electrical interfaces commonly constitute the foundation of integrated systems for the closed-loop control of neural function. This is partially due to the wide use of both electrical monitoring and modulation in currently implemented devices, along 


\section{WILEY-VCH}

with its compatibility with microfabrication techniques. Electrical monitoring provides a good balance between spatiotemporal resolution and device footprint, and therefore has become the main modality to monitor neural tissue in multifunctional devices. Electrical monitoring interfaces can easily be adapted to produce an electrical signal for the modulation aspect of a multifunctional device. This configuration has led to promising implementations as a feedback control for modulation ${ }^{[50,298,299]}$. However, while electrical modulation provides similar benefits as electrical monitoring in terms of spatiotemporal resolution and size, when combined with electrical monitoring techniques, the electrical stimulation leads to artifacts in the recording during and immediately following the stimulation, which introduces a latency in the closedloop control ${ }^{[298,300]}$ (Figure 16A). Therefore, a majority of multifunctional devices pair chemical or optical modulation with electrical monitoring. There are several examples that utilize electrical monitoring as a feedback signal to release a chemical modulator in response to a pathologic electrophysiological signal (Figure 16B and E) ${ }^{[88,221,301-303]}$, or incorporate a chemical modulating interface to release anti-inflammatory drugs to reduce the chronic immune response and increase device longevity ${ }^{[203,205,206]}$. There are also many examples of neural implants that couple electrical monitoring with optical stimulation ${ }^{[260,304-308]}$ (Figure 16C and D). These technologies typically revolve around utilizing optogenetic stimulation with electrophysiological feedback, and are useful in many fundamental neuroscience studies. However, with the advent of new gene editing strategies, new avenues for the treatment of diseases can be imagined ${ }^{[1,309]}$. While it is also feasible to utilize electrical modulation with other modalities such as optical and chemical monitoring for the feedback signal, this is less common $^{[103,310]}$. In one system, the monitoring is done via extracellular electrophysiology (electrical recording) and voltammetry (neurotransmitter detection), and the stimulation is carried out via iontophoresis of neuromodulators ${ }^{[311]}$. For optical monitoring, a challenge is electrode opacity. Materials such as indium tin oxide are commonly used for in vitro implementations, but they lack the benefits of the nanostructured surfaces that improve the 


\section{WILEY-VCH}

performance of electrical monitoring interfaces. However, some successful implementations of optical monitoring have been achieved through combination with chemical or optical modulation $^{[7,312-314]}$.

Ultimately, the most effective way to create a multifunctional neural implant is to combine two or more existing modalities into a single device. Fabrication compatibility becomes the limiting factor in this scenario. For devices containing optical fibers or cannulas, fabrication techniques compatible with these materials, such as thermal drawing ${ }^{[304]}$ and metallization of an optical fiber cladding ${ }^{[315]}$, are necessary. For example, a thermal drawing process was used to fabricate a multifunctional fiber, which was capable of optical and chemical modulation through optical waveguides and microfluidic channels respectively, and electrical monitoring through conductive polymer electrodes $^{[304]}$ (Figure 16C). While these devices are relatively simple and economical (thus accessible by researcher) to fabricate, each fiber is only capable of interrogating a small volume of neural tissue, the formation of an array of these fibers is time consuming, and individual devices are not reproducible (as each device is typically made manually). Alternatively, by using microfabrication techniques, multifunctional devices can be reproducibly fabricated in a variety of combinations and complex geometries. Some examples are Michigan-style probes fabricated from SU8 containing $20 \mu \mathrm{m}$-diameter platinum recording electrodes with embedded microfluidic channels ${ }^{[221]}$ and a Utah-style array consisting of microneedle SU8 waveguides with a metal/polyimide cladding that also functions as recording electrodes $^{[266]}$ (Figure 16D and E). However, while these strategies produce multifunctional devices, they typically do not embody multifunctional interfaces, as each individual interface only consists of a single modality. 


\section{WILEY-VCH}

As multifunctional neural implants are typically designed for long term applications, device failure due to the chronic immune response becomes a larger issue. Therefore the ability to fabricate a multifunctional interface in the smallest footprint possible is not only important for improving spatial resolution, but also for minimizing the inflammatory response ${ }^{[316]}$. To that end interface materials that are capable of both monitoring and modulating neural activity such as np-Au, carbon fibers, and conductive polymers are particularly well suited to multifunctional applications. Additionally, all three have shown compatibility with microfabrication processes and the ability to be incorporated into compliant substrates ${ }^{[317-320]}$. An excellent example of the implementation of a multifunctional neural interface is the 'bioelectronic neural pixel' ${ }^{\text {'[88] }}$ (Figure 12B and Figure 16B). In this example, an electrophysiological recording of a neural tissue slice is used as the feedback signal for triggered release of an inhibitory neurotransmitter, GABA, from a polymer reservoir onto the tissue slice, effectively utilizing the nanostructured conducting polymer to facilitate this multifunctionality at a single electrode footprint.

\section{Outlook \& Conclusion}

In this article, we have reviewed several strategies for monitoring and modulating neural activity. While the progress on this front is expected to continue, a new front is the multifunctional and closed-loop control of neural systems. This is a multifaceted problem that involves not only innovations on the devices and materials front, but also on the controls, communications, power transfer, and thermal management. For the former, creating devices with integrated optical, fluidic, electrical components with high yield and reliability will require new fabrication strategies, especially if the components were to be incorporated on soft substrates, such as polyimide, SU8, parylene-C, and silicone ${ }^{[321]}$. Advancements have also been made towards realizing true wireless control (both input and output) from an implanted device through ultrasonic waves ${ }^{[322]}$. Another challenge is controlling the modulation, especially via soluble cues. A significant issue for any drug delivery platform is the drug cargo eventually 


\section{WILEY-VCH}

depleting. There have been interesting examples of refilling cargo using nucleic acid-based carriers $^{[323]}$ and recycling neurotransmitters for sustained cargo ${ }^{[324]}$. In order to efficiently use the limited storage capacity in miniaturized systems, potent neuromodulators and high concentrations are used, which necessitates precise dosing of the active molecules. Accordingly, there is a need for actuation and control algorithms to attain this. The control algorithm development and systems identification concept described in the previous section faces the significant challenge of how to treat neural systems that are far from static (i.e., everevolving via their inherent plasticity). To that end, there is no doubt that new theoretical frameworks are needed to be able to accurately develop dynamic transfer functions for such systems. It is also crucial to underscore the importance regulatory considerations in translating neural interface technologies to patients, which will in the end determine whether a technology has true clinical value ${ }^{[325,326]}$.

Additionally, with the ever-expanding repertoire of multifunctional materials, a need has emerged for high-throughput platforms to rapidly assess material attributes and their influence on device function. As discussed previously, there have been many innovations on this front with both in vitro and in vivo combinatorial systems but with a very limited focus on neural applications. Further translation of these combinatorial material characterization techniques to neural interfaces and subsequently to combinatorial studies in vivo will significantly accelerate advancements in the development of new material coatings. Additionally, translating these studies to other material dependent areas such as the development of new device substrates from materials such as bioactive or smart polymers can further improve the success of implanted devices. Ultimately, it is expected that the development of multifunctional systems will prove to be essential for the treatment of neurological diseases and that through improving device functionality it will be possible to achieve the long-standing goal of a single chronic therapeutic implant. 


\section{WILEY-VCH}

\section{Conflict of Interest}

The authors declare no conflict of interest.

\section{Acknowledgements}

We gratefully acknowledge the support from National Science Foundation Awards (CBET1512745 and CBET\&DMR-1454426).

Received: ((will be filled in by the editorial staff))

Revised: ((will be filled in by the editorial staff)) Published online: ((will be filled in by the editorial staff)) 


\section{WILEY-VCH}

\section{References}

[1] E. Krook-Magnuson, C. Armstrong, M. Oijala, I. Soltesz, Nature Communications 2013, 4, 1376.

[2] R. Chen, A. Canales, P. Anikeeva, Nature Reviews Materials 2017, 2, natrevmats201693.

[3] A. H. Marblestone, B. M. Zamft, Y. G. Maguire, M. G. Shapiro, T. R. Cybulski, J. I. Glaser, D. Amodei, P. B. Stranges, R. Kalhor, D. A. Dalrymple, D. Seo, E. Alon, M. M. Maharbiz, J. M. Carmena, J. M. Rabaey, E. S. Boyden, G. M. Church, K. P. Kording, Front Comput Neurosci 2013, 7, 137.

[4] S. F. Cogan, Annu Rev Biomed Eng 2008, 10, 275.

[5] D. L. Robinson, A. Hermans, A. T. Seipel, R. M. Wightman, Chem. Rev. 2008, 108, 2554.

[6] A. M. Aravanis, L.-P. Wang, F. Zhang, L. A. Meltzer, M. Z. Mogri, M. B. Schneider, K. Deisseroth, J Neural Eng 2007, 4, S143.

[7] A. M. Packer, L. E. Russell, H. W. P. Dalgleish, M. Häusser, Nature Methods 2015, 12,140 .

[8] W. Legon, T. F. Sato, A. Opitz, J. Mueller, A. Barbour, A. Williams, W. J. Tyler, Nat. Neurosci. 2014, 17, 322.

[9] E. Macé, G. Montaldo, I. Cohen, M. Baulac, M. Fink, M. Tanter, Nature Methods 2011, 8, 662 .

[10] E. A. Allen, B. N. Pasley, T. Duong, R. D. Freeman, Science 2007, 317, 1918.

[11] S. Khan, G. Newaz, J Biomed Mater Res A 2010, 93, 1209.

[12] M. Goldberg, R. Langer, X. Jia, J Biomater Sci Polym Ed 2007, 18, 241.

[13] R. T. Kennedy, Curr Opin Chem Biol 2013, 17, 860.

[14] E. S. Bucher, R. M. Wightman, Annu Rev Anal Chem (Palo Alto Calif) 2015, 8, 239.

[15] L. Fenno, O. Yizhar, K. Deisseroth, Annu. Rev. Neurosci. 2011, 34, 389.

[16] M. Mank, O. Griesbeck, Chem. Rev. 2008, 108, 1550.

[17] S. A. Hires, L. Tian, L. L. Looger, Brain Cell Biology 2008, 36, 69.

[18] T. Knöpfel, Nat. Rev. Neurosci. 2012, 13, 687.

[19] L. L. Looger, O. Griesbeck, Curr. Opin. Neurobiol. 2012, 22, 18.

[20] W. Yang, R. Yuste, Nature Methods 2017, 14, 349.

[21] B. A. Wilt, L. D. Burns, E. T. Wei Ho, K. K. Ghosh, E. A. Mukamel, M. J.

Schnitzer, Annu. Rev. Neurosci. 2009, 32, 435.

[22] C. K. Kim, A. Adhikari, K. Deisseroth, Nat. Rev. Neurosci. 2017, 18, 222.

[23] K. Huang, Q. Dou, X. J. Loh, RSC Adv. 2016, 6, 60896.

[24] P. Fattahi, G. Yang, G. Kim, M. R. Abidian, Adv. Mater. Weinheim 2014, 26, 1793.

[25] W. M. Grill, S. E. Norman, R. V. Bellamkonda, Annu Rev Biomed Eng 2009, 11, 1.

[26] N. A. Kotov, J. O. Winter, I. P. Clements, E. Jan, B. P. Timko, S. Campidelli, S.

Pathak, A. Mazzatenta, C. M. Lieber, M. Prato, R. V. Bellamkonda, G. A. Silva, N.

W. S. Kam, F. Patolsky, L. Ballerini, Adv. Mater. Weinheim 2009, 21, 3970.

[27] D. A. Robinson, Proceedings of the IEEE 1968, 56, 1065.

[28] M. E. Spira, A. Hai, Nature Nanotechnology 2013, 8, 83.

[29] W. Franks, I. Schenker, P. Schmutz, A. Hierlemann, IEEE Transactions on Biomedical Engineering 2005, 52, 1295.

[30] M. Jorfi, J. L. Skousen, C. Weder, J. R. Capadona, J Neural Eng 2015, 12.

[31] M. P. Ward, P. Rajdev, C. Ellison, P. P. Irazoqui, Brain Res. 2009, 1282, 183.

[32] M. A. Long, A. K. Lee, Curr. Opin. Neurobiol. 2012, 22, 34.

[33] V. Marx, Nature Methods 2014, 11, 1099.

[34] M. R. Angle, B. Cui, N. A. Melosh, Curr. Opin. Neurobiol. 2015, 32, 132. 


\section{WILEY-VCH}

[35] S. WOLDRING, M. N. J. DIRKEN, Acta Physiol Pharmacol Neerl 1950, 1, 369.

[36] R. M. DOWBEN, J. E. ROSE, Science 1953, 118, 22.

[37] C. W. Sem-Jacobsen, J. Neurosurg. 1966, 24, Suppl:388.

[38] J. M. R. Delgado, H. Hamlin, W. P. Chapman, Stereotactic and Functional Neurosurgery 1952, 12, 315.

[39] K. D. Harris, D. A. Henze, J. Csicsvari, H. Hirase, G. Buzsaki, J. Neurophysiol. 2000, 84, 401.

[40] F. Pisanello, L. Sileo, M. De Vittorio, Front Neurosci 2016, 10, 70.

[41] G. Buzsáki, Nat. Neurosci. 2004, 7, 446.

[42] M. P. Ward, P. Rajdev, C. Ellison, P. P. Irazoqui, Brain Res. 2009, 1282, 183.

[43] D. R. Kipke, W. Shain, G. Buzsaki, E. Fetz, J. M. Henderson, J. F. Hetke, G. Schalk, Journal of Neuroscience 2008, 28, 11830.

[44] V. S. Polikov, P. A. Tresco, W. M. Reichert, J. Neurosci. Methods 2005, 148, 1.

[45] A. J. Woolley, H. A. Desai, K. J. Otto, J Neural Eng 2013, 10, 026007.

[46] J. Pine, J. Neurosci. Methods 1980, 2, 19.

[47] K. G. Shah, V. M. Tolosa, A. C. Tooker, S. H. Felix, S. S. Pannu, Conf Proc IEEE Eng Med Biol Soc 2013, 2013, 1546.

[48] S. Negi, R. Bhandari, L. Rieth, F. Solzbacher, Biomed Mater 2010, 5, 15007.

[49] Y. Rui, J. Liu, Y. Wang, C. Yang, Microsystem Technologies-Micro-and Nanosystems-Information Storage and Processing Systems 2011, 17, 437.

[50] D. C. Rodger, A. J. Fong, W. Li, H. Ameri, A. K. Ahuja, C. Gutierrez, I. Lavrov, H. Zhong, P. R. Menon, E. Meng, J. W. Burdick, R. R. Roy, V. R. Edgerton, J. D. Weiland, M. S. Humayun, Y.-C. Tai, Sensors and Actuators B: Chemical 2008, 132, 449.

[51] B. Ilic, D. Czaplewski, P. Neuzil, T. Stanczyk, J. Blough, G. J. Maclay, Journal of Materials Science 2000, 35, 3447.

[52] S. F. Cogan, T. D. Plante, J. Ehrlich, Conf Proc IEEE Eng Med Biol Soc 2004, 6, 4153.

[53] J. D. Weiland, D. J. Anderson, M. S. Humayun, IEEE Transactions on Biomedical Engineering 2002, 49, 1574.

[54] C. A. R. Chapman, L. Wang, H. Chen, J. Garrison, P. J. Lein, E. Seker, Advanced Functional Materials 2016, 1604631.

[55] E. Seker, Y. Berdichevsky, M. R. Begley, M. L. Reed, K. J. Staley, M. L. Yarmush, Nanotechnology 2010, 21, 125504.

[56] D. Brüggemann, B. Wolfrum, V. Maybeck, Y. Mourzina, M. Jansen, A. Offenhäusser, Nanotechnology 2011, 22, 265104.

[57] H. Yoon, D. C. Deshpande, V. Ramachandran, V. K. Varadan, Nanotechnology 2008, 19 .

[58] C. Boehler, T. Stieglitz, M. Asplund, Biomaterials 2015, 67, 346.

[59] J.-H. Kim, G. Kang, Y. Nam, Y.-K. Choi, Nanotechnology 2010, 21, 85303.

[60] Y. H. Kim, G. H. Kim, A. Y. Kim, Y. H. Han, M.-A. Chung, S.-D. Jung, J Neural Eng 2015, 12, 066029.

[61] V. Vogel, M. Sheetz, Nat. Rev. Mol. Cell Biol. 2006, 7, 265.

[62] M. J. P. Biggs, R. G. Richards, M. J. Dalby, Nanomedicine 2010, 6, 619.

[63] H. K. Kim, E. Kim, H. Jang, Y.-K. Kim, K. Kang, ChemNanoMat 2017, 3, 278.

[64] B. K. K. Teo, S. T. Wong, C. K. Lim, T. Y. S. Kung, C. H. Yap, Y. Ramagopal, L. H. Romer, E. K. F. Yim, ACS Nano 2013, 7, 4785.

[65] K. Yang, K. Jung, E. Ko, J. Kim, K. I. Park, J. Kim, S.-W. Cho, ACS Appl Mater Interfaces 2013, 5, 10529.

[66] V. S. Polikov, M. L. Block, J.-M. Fellous, J.-S. Hong, W. M. Reichert, Biomaterials 2006, 27, 5368 . 


\section{WILEY-VCH}

[67] M. V. Sofroniew, H. V. Vinters, Acta Neuropathol. 2010, 119, 7.

[68] M. Pekny, M. Pekna, Physiol. Rev. 2014, 94, 1077.

[69] V. Brunetti, G. Maiorano, L. Rizzello, B. Sorce, S. Sabella, R. Cingolani, P. P. Pompa, Proc. Natl. Acad. Sci. U.S.A. 2010, 107, 6264.

[70] A. Ferrari, M. Cecchini, A. Dhawan, S. Micera, I. Tonazzini, R. Stabile, D. Pisignano, F. Beltram, Nano Lett. 2011, 11, 505.

[71] Y. H. Tan, S. E. Terrill, G. S. Paranjape, K. J. Stine, M. R. Nichols, Biomater. Sci. 2014, 2, 110.

[72] C. A. R. Chapman, H. Chen, M. Stamou, J. Biener, M. M. Biener, P. J. Lein, E. Seker, ACS Appl Mater Interfaces 2015, 7, 7093.

[73] J. P. Spatz, B. Geiger, Methods Cell Biol. 2007, 83, 89.

[74] M. Schvartzman, M. Palma, J. Sable, J. Abramson, X. Hu, M. P. Sheetz, S. J. Wind, Nano Lett. 2011, 11, 1306.

[75] K. Yang, K. Jung, E. Ko, J. Kim, K. I. Park, J. Kim, S.-W. Cho, ACS Appl Mater Interfaces 2013, 5, 10529.

[76] E. S. Ereifej, H. W. Matthew, G. Newaz, A. Mukhopadhyay, G. Auner, I. Salakhutdinov, P. J. VandeVord, J Biomed Mater Res A 2013, 101, 1743.

[77] N. Tawil, P. Wilson, S. Carbonetto, The Journal of Cell Biology 1993, 120, 261.

[78] S. P. Lacour, G. Courtine, J. Guck, Nature Reviews Materials 2016, 1, 16063.

[79] C. L. Kolarcik, S. D. Luebben, S. A. Sapp, J. Hanner, N. Snyder, T. D. Y. Kozai, E. Chang, J. A. Nabity, S. T. Nabity, C. F. Lagenaur, X. T. Cui, Soft Matter 2015, 11, 4847.

[80] H. Han, A. M. Reichmuth, A. F. Renz, F. Stauffer, M. Thielen, J. Vörös, International Journal of Automation and Smart Technology 2017, 7, 37.

[81] R. Green, M. R. Abidian, Adv. Mater. Weinheim 2015, 27, 7620.

[82] X. Cui, D. C. Martin, Sensors and Actuators B: Chemical 2003, 89, 92.

[83] K. A. Ludwig, J. D. Uram, J. Yang, D. C. Martin, D. R. Kipke, J Neural Eng 2006, 3,59 .

[84] R. A. Green, N. H. Lovell, G. G. Wallace, L. A. Poole-Warren, Biomaterials 2008, 29, 3393.

[85] M. R. Abidian, K. A. Ludwig, T. C. Marzullo, D. C. Martin, D. R. Kipke, $A d v$. Mater. Weinheim 2009, 21, 3764.

[86] M. R. Abidian, D. C. Martin, Biomaterials 2008, 29, 1273.

[87] X. Cui, V. A. Lee, Y. Raphael, J. A. Wiler, J. F. Hetke, D. J. Anderson, D. C. Martin, J. Biomed. Mater. Res. 2001, 56, 261.

[88] A. Jonsson, S. Inal, L. Uguz, A. J. Williamson, L. Kergoat, J. Rivnay, D. Khodagholy, M. Berggren, C. Bernard, G. G. Malliaras, D. T. Simon, Proc. Natl. Acad. Sci. U.S.A. 2016, 113, 9440.

[89] E. W. Keefer, B. R. Botterman, M. I. Romero, A. F. Rossi, G. W. Gross, Nature Nanotechnology 2008, 3, 434.

[90] T. Gabay, M. Ben-David, I. Kalifa, R. Sorkin, Z. R. Abrams, E. Ben-Jacob, Y. Hanein, Nanotechnology 2007, 18.

[91] M. David-Pur, L. Bareket-Keren, G. Beit-Yaakov, D. Raz-Prag, Y. Hanein, Biomedical Microdevices 2014, 16, 43.

[92] H.-C. Su, C.-M. Lin, S.-J. Yen, Y.-C. Chen, C.-H. Chen, S.-R. Yeh, W. Fang, H. Chen, D.-J. Yao, Y.-C. Chang, T.-R. Yew, Biosens Bioelectron 2010, 26, 220.

[93] K. Fuchsberger, A. Le Goff, L. Gambazzi, F. M. Toma, A. Goldoni, M. Giugliano, M. Stelzle, M. Prato, Small 2011, 7, 524.

[94] J. L. McKenzie, M. C. Waid, R. Shi, T. J. Webster, Biomaterials 2004, 25, 1309.

[95] T. J. Webster, M. C. Waid, J. L. McKenzie, R. L. Price, J. U. Ejiofor, Nanotechnology 2003, 15, 48. 


\section{WILEY-VCH}

[96] M. P. Mattson, R. C. Haddon, A. M. Rao, J. Mol. Neurosci. 2000, 14, 175.

[97] D.-W. Park, S. K. Brodnick, J. P. Ness, F. Atry, L. Krugner-Higby, A. Sandberg, S. Mikael, T. J. Richner, J. Novello, H. Kim, D.-H. Baek, J. Bong, S. T. Frye, S. Thongpang, K. I. Swanson, W. Lake, R. Pashaie, J. C. Williams, Z. Ma, Nature Protocols 2016, 11, 2201.

[98] Y. Lu, H. Lyu, A. G. Richardson, T. H. Lucas, D. Kuzum, Sci Rep 2016, 6, 33526.

[99] S. W. Hong, J. H. Lee, S. H. Kang, E. Y. Hwang, Y.-S. Hwang, M. H. Lee, D.-W. Han, J.-C. Park, Biomed Res Int 2014, 2014, 212149.

[100] M. Lorenzoni, F. Brandi, S. Dante, A. Giugni, B. Torre, Sci Rep 2013, 3, 1954.

[101] D. Khodagholy, T. Doublet, P. Quilichini, M. Gurfinkel, P. Leleux, A. Ghestem, E. Ismailova, T. Hervé, S. Sanaur, C. Bernard, G. G. Malliaras, Nature

Communications 2013, 4, ncomms2573.

[102] L. Yang, Y. Zhao, W. Xu, E. Shi, W. Wei, X. Li, A. Cao, Y. Cao, Y. Fang, Nano Lett. 2017, 17, 71.

[103] V. Benfenati, S. Toffanin, S. Bonetti, G. Turatti, A. Pistone, M. Chiappalone, A. Sagnella, A. Stefani, G. Generali, G. Ruani, D. Saguatti, R. Zamboni, M. Muccini, Nature Materials 2013, 12, 672.

[104] M. Borisovska, A. L. Bensen, G. Chong, G. L. Westbrook, J. Neurosci. 2013, 33, 1790.

[105] X. Borue, S. Cooper, J. Hirsh, B. Condron, B. J. Venton, J. Neurosci. Methods 2009, 179, 300.

[106] J. Park, P. Takmakov, R. M. Wightman, J. Neurochem. 2011, 119, 932.

[107] E. A. Kiyatkin, K. T. Wakabayashi, M. Lenoir, ACS Chem Neurosci 2013, 4, 652.

[108] J. Petrovic, P. L. Walsh, K. T. Thornley, C. E. Miller, R. M. Wightman, Endocrinology 2010, 151, 1773.

[109] R. H. S. Westerink, A. G. Ewing, Acta Physiol (Oxf) 2008, 192, 273.

[110] J. G. Osteryoung, R. A. Osteryoung, Anal. Chem. 1985, 57, 101A.

[111] K. L. Davis, R. S. Kahn, G. Ko, M. Davidson, Am J Psychiatry 1991, 148, 1474.

[112] H. Bernheimer, W. Birkmayer, O. Hornykiewicz, K. Jellinger, F. Seitelberger, J. Neurol. Sci. 1973, 20, 415.

[113] B. E. K. Swamy, B. J. Venton, Anal. Chem. 2007, 79, 744.

[114] M. L. Pajski, B. J. Venton, Purinergic Signal. 2013, 9, 167.

[115] A. C. Schmidt, L. E. Dunaway, J. G. Roberts, G. S. McCarty, L. A. Sombers, Anal. Chem. 2014, 86, 7806.

[116] R. N. Adams, Anal. Chem. 1976, 48, 1126A.

[117] T. M. Squires, R. J. Messinger, S. R. Manalis, Nat. Biotechnol. 2008, 26, 417.

[118] P. E. Sheehan, L. J. Whitman, Nano Lett. 2005, 5, 803.

[119] P. R. Nair, M. A. Alam, Applied Physics Letters 2006, 88, 233120.

[120] A. L. Sanford, S. W. Morton, K. L. Whitehouse, H. M. Oara, L. Z. Lugo-Morales, J. G. Roberts, L. A. Sombers, Anal. Chem. 2010, 82, 5205.

[121] M. L. Huffman, B. J. Venton, Analyst 2008, 134, 18.

[122] A. H. Swiergiel, V. S. Palamarchouk, A. J. Dunn, J. Neurosci. Methods 1997, 73, 29.

[123] S. Ranganathan, R. McCreery, S. M. Majji, M. Madou, Journal of The Electrochemical Society 2000, 147, 277.

[124] S. O. Kelley, C. A. Mirkin, D. R. Walt, R. F. Ismagilov, M. Toner, E. H. Sargent, Nature Nanotechnology 2014, 9, 969.

[125] P. Daggumati, Z. Matharu, L. Wang, E. Seker, Anal. Chem. 2015, 87, 8618.

[126] J. Patel, L. Radhakrishnan, B. Zhao, B. Uppalapati, R. C. Daniels, K. R. Ward, M. M. Collinson, Anal. Chem. 2013, 85, 11610.

[127] S. Saraf, C. J. Neal, S. Park, S. Das, S. Barkam, H. J. Cho, S. Seal, RSC Adv. 2015, 


\section{WILEY-VCH}

5,46501 .

[128] D. A. Zhang, E. Rand, M. Marsh, R. J. Andrews, K. H. Lee, M. Meyyappan, J. E. Koehne, Mol. Neurobiol. 2013, 48, 380.

[129] C. B. Jacobs, M. J. Peairs, B. J. Venton, Anal. Chim. Acta 2010, 662, 105.

[130] Y. Wang, Y. Li, L. Tang, J. Lu, J. Li, Electrochemistry Communications 2009, 11, 889.

[131] H.-J. Qiu, G.-P. Zhou, G.-L. Ji, Y. Zhang, X.-R. Huang, Y. Ding, Colloids Surf B Biointerfaces 2009, 69, 105.

[132] E. Kätelhön, B. Hofmann, S. G. Lemay, M. A. G. Zevenbergen, A. Offenhäusser, B. Wolfrum, Anal. Chem. 2010, 82, 8502.

[133] E. Kätelhön, B. Wolfrum, Reviews in Analytical Chemistry 2012, 31.

[134] H. R. Zafarani, K. Mathwig, E. J. R. Sudhölter, L. Rassaei, ACS Sensors 2017, 2, 724.

[135] W. H. Oldenziel, Ben H C Westerink, Anal. Chem. 2005, 77, 5520.

[136] M. Sarter, V. Parikh, W. M. Howe, Nat. Rev. Neurosci. 2009, 10, 383.

[137] L. I. Schmitt, R. E. Sims, N. Dale, P. G. Haydon, J. Neurosci. 2012, 32, 4417.

[138] I. M. Taylor, Z. Du, E. T. Bigelow, J. R. Eles, A. R. Horner, K. A. Catt, S. G.

Weber, B. G. Jamieson, X. T. Cui, J. Mater. Chem. B 2017, 5, 2445.

[139] W. H. Lee, T. Ngernsutivorakul, O. S. Mabrouk, J.-M. T. Wong, C. E. Dugan, S. S. Pappas, H. J. Yoon, R. T. Kennedy, Anal. Chem. 2016, 88, 1230.

[140] P. M. Vespa, D. McArthur, K. O'Phelan, T. Glenn, M. Etchepare, D. Kelly, M. Bergsneider, N. A. Martin, D. A. Hovda, J. Cereb. Blood Flow Metab. 2003, 23, 865.

[141] A. W. Bero, P. Yan, J. H. Roh, J. R. Cirrito, F. R. Stewart, M. E. Raichle, J.-M. Lee, D. M. Holtzman, Nat. Neurosci. 2011, 14, 750.

[142] T. R. Slaney, J. Nie, N. D. Hershey, P. K. Thwar, J. Linderman, M. A. Burns, R. T. Kennedy, Anal. Chem. 2011, 83, 5207.

[143] F. Gu, X. Zhou, X. Zhu, M. Zhao, J. Hao, P. Yu, L. Mao, Analyst 2015, 140, 3814.

[144] M. Wang, G. T. Roman, K. Schultz, C. Jennings, R. T. Kennedy, Anal. Chem. 2008, $80,5607$.

[145] M. J. de Boer, R. W. Tjerkstra, J. W. Berenschot, H. V. Jansen, G. J. Burger, J. G.

E. Gardeniers, M. Elwenspoek, A. van den Berg, Journal of

Microelectromechanical Systems 2000, 9, 94.

[146] S. Kottegoda, I. Shaik, S. A. Shippy, J. Neurosci. Methods 2002, 121, 93.

[147] T. R. Slaney, O. S. Mabrouk, K. A. Porter-Stransky, B. J. Aragona, R. T. Kennedy, ACS Chem Neurosci 2013, 4, 321.

[148] P. Song, N. D. Hershey, O. S. Mabrouk, T. R. Slaney, R. T. Kennedy, Anal. Chem. 2012, 84, 4659.

[149] S. Song, A. K. Singh, T. J. Shepodd, B. J. Kirby, Anal. Chem. 2004, 76, 2367.

[150] Bin Liu, H. J. Lee, D. Zhang, C.-S. Liao, N. Ji, Y. Xia, J.-X. Cheng, Applied Physics Letters 2015, 106, 173704.

[151] S. Oh, C. Fang-Yen, W. Choi, Z. Yaqoob, D. Fu, Y. Park, R. R. Dassari, M. S. Feld, Biophys. J. 2012, 103, 11.

[152] Y. Garini, A. Gil, I. Bar-Am, D. Cabib, N. Katzir, Cytometry Part A 1999, 35, 214.

[153] M. Scanziani, M. Häusser, Nature 2009, 461, 930.

[154] W. Denk, K. R. Delaney, A. Gelperin, D. Kleinfeld, B. W. Strowbridge, D. W. Tank, R. Yuste, J. Neurosci. Methods 1994, 54, 151.

[155] M. Chalfie, Y. Tu, G. Euskirchen, W. W. Ward, D. C. Prasher, Science 1994, 263, 802.

[156] S. Higashijima, Y. Hotta, H. Okamoto, J. Neurosci. 2000, 20, 206.

[157] A. L. Peel, S. Zolotukhin, G. W. Schrimsher, N. Muzyczka, P. J. Reier, Gene Ther. 


\section{WILEY-VCH}

1997, 4, 16.

[158] G. Feng, R. H. Mellor, M. Bernstein, C. Keller-Peck, Q. T. Nguyen, M. Wallace, J. M. Nerbonne, J. W. Lichtman, J. R. Sanes, Neuron 2000, 28, 41.

[159] J. Priller, A. Flügel, T. Wehner, M. Boentert, C. A. Haas, M. Prinz, F. FernándezKlett, K. Prass, I. Bechmann, B. A. de Boer, M. Frotscher, G. W. Kreutzberg, D. A. Persons, U. Dirnagl, Nature Medicine 2001, 7, 1356.

[160] M. M. Halassa, T. Fellin, H. Takano, J.-H. Dong, P. G. Haydon, J. Neurosci. 2007, 27, 6473.

[161] J. Livet, T. A. Weissman, H. Kang, R. W. Draft, J. Lu, R. A. Bennis, J. R. Sanes, J. W. Lichtman, Nature 2007, 450, 56.

[162] D. Cai, K. B. Cohen, T. Luo, J. W. Lichtman, J. R. Sanes, Nature Methods 2013, 10,540 .

[163] D. Gomez-Nicola, K. Riecken, B. Fehse, V. H. Perry, Sci Rep 2014, 4, 7520.

[164] F. St-Pierre, J. D. Marshall, Y. Yang, Y. Gong, M. J. Schnitzer, M. Z. Lin, Nat. Neurosci. 2014, 17, 884.

[165] V. Emiliani, A. E. Cohen, K. Deisseroth, M. Häusser, J. Neurosci. 2015, 35, 13917.

[166] R. Schneggenburger, E. Neher, Nature 2000, 406, 889.

[167] D. Lipscombe, D. V. Madison, M. Poenie, H. Reuter, R. Y. Tsien, R. W. Tsien, Proc. Natl. Acad. Sci. U.S.A. 1988, 85, 2398.

[168] J. H. Goldberg, R. Yuste, G. Tamas, The Journal of Physiology 2003, 551, 67.

[169] C. Grienberger, A. Konnerth, Neuron 2012, 73, 862.

[170] T.-W. Chen, T. J. Wardill, Y. Sun, S. R. Pulver, S. L. Renninger, A. Baohan, E. R. Schreiter, R. A. Kerr, M. B. Orger, V. Jayaraman, L. L. Looger, K. Svoboda, D. S. Kim, Nature 2013, 499, 295.

[171] J. W. Lichtman, J.-A. Conchello, Nature Methods 2005, 2, 910.

[172] M. J. Pittet, R. Weissleder, Cell 2011, 147, 983.

[173] K. Carlsson, P. E. Danielsson, R. Lenz, A. Liljeborg, L. Majlöf, N. Aslund, Opt Lett 1985, 10, 53.

[174] P. Theer, M. T. Hasan, W. Denk, Opt Lett 2003, 28, 1022.

[175] F. Helmchen, W. Denk, Nature Methods 2005, 2, 932.

[176] A. Cheng, J. T. Gonçalves, P. Golshani, K. Arisaka, C. Portera-Cailliau, Nature Methods 2011, 8, 139.

[177] P. Mahou, M. Zimmerley, K. Loulier, K. S. Matho, G. Labroille, X. Morin, W. Supatto, J. Livet, D. Débarre, E. Beaurepaire, Nature Methods 2012, 9, 815.

[178] F. Anselmi, C. Ventalon, A. Begue, D. Ogden, V. Emiliani, Proceedings of the National Academy of Sciences 2011, 108, 19504.

[179] A. Birkner, C. H. Tischbirek, A. Konnerth, Cell Calcium 2017, 64, 29.

[180] N. G. Horton, K. Wang, D. Kobat, C. G. Clark, F. W. Wise, C. B. Schaffer, C. Xu, Nat Photonics 2013, 7, 205.

[181] W. Mittmann, D. J. Wallace, U. Czubayko, J. T. Herb, A. T. Schaefer, L. L. Looger, W. Denk, J. N. D. Kerr, Nat. Neurosci. 2011, 14, 1089.

[182] A. Holtmaat, T. Bonhoeffer, D. K. Chow, J. Chuckowree, V. De Paola, S. B. Hofer, M. Hübener, T. Keck, G. Knott, W.-C. A. Lee, R. Mostany, T. D. Mrsic-Flogel, E. Nedivi, C. Portera-Cailliau, K. Svoboda, J. T. Trachtenberg, L. Wilbrecht, Nature Protocols 2009, 4, 1128.

[183] P. J. Drew, A. Y. Shih, J. D. Driscoll, P. M. Knutsen, P. Blinder, D. Davalos, K. Akassoglou, P. S. Tsai, D. Kleinfeld, Nature Methods 2010, 7, 981.

[184] T. H. Chia, M. J. Levene, J. Neurophysiol. 2009, 102, 1310.

[185] M. L. Andermann, N. B. Gilfoy, G. J. Goldey, R. N. S. Sachdev, M. Wölfel, D. A. McCormick, R. C. Reid, M. J. Levene, Neuron 2013, 80, 900.

[186] M. J. Levene, D. A. Dombeck, K. A. Kasischke, R. P. Molloy, W. W. Webb, J. 


\section{WILEY-VCH}

Neurophysiol. 2004, 91, 1908.

[187] J. C. Jung, A. D. Mehta, E. Aksay, R. Stepnoski, M. J. Schnitzer, J. Neurophysiol. 2004, 92, 3121.

[188] J. Knittel, L. Schnieder, G. Buess, B. Messerschmidt, T. Possner, Optics Communications 2001, 188, 267.

[189] C. Wang, N. Ji, Opt Express 2013, 21, 27142.

[190] M. E. Bocarsly, W.-C. Jiang, C. Wang, J. T. Dudman, N. Ji, Y. Aponte, Biomedical Optics Express 2015, 6, 4546.

[191] B. A. Flusberg, E. D. Cocker, W. Piyawattanametha, J. C. Jung, E. L. M. Cheung, M. J. Schnitzer, Nature Methods 2005, 2, 941.

[192] D. C. Ng, T. Nakagawa, T. Mizuno, T. Tokuda, M. Nunoshita, H. Tamura, Y. Ishikawa, S. Shiosaka, J. Ohta, IEEE Sensors Journal 2008, 8, 121.

[193] D. R. Merrill, M. Bikson, J. Jefferys, J. Neurosci. Methods 2005, 141, 171.

[194] K. C. Cheung, Biomedical Microdevices 2007, 9, 923.

[195] S. F. Cogan, J. Ehrlich, T. D. Plante, A. Smirnov, D. B. Shire, M. Gingerich, J. F. Rizzo, J. Biomed. Mater. Res. Part B Appl. Biomater. 2009, 89, 353.

[196] C. Outen, D. W. Konopka, T. F. Fennessey, Society of Vacuum Coaters, 2014; Vol. 57, pp. 33-37.

[197] U. Egert, B. Schlosshauer, S. Fennrich, W. Nisch, M. Fejtl, T. Knott, T. Muller, H. Hammerle, Brain Res. Brain Res. Protoc. 1998, 2, 229.

[198] K. Wang, H. A. Fishman, H. Dai, J. S. Harris, Nano Lett. 2006, 6, 2043.

[199] X. T. Cui, D. D. Zhou, IEEE Trans Neural Syst Rehabil Eng 2007, 15, 502.

[200] Y. H. Kim, G. H. Kim, M. S. Kim, S.-D. Jung, Nano Lett. 2016, 16, 7163.

[201] P. R. Lockman, R. J. Mumper, M. A. Khan, D. D. Allen, Drug Dev Ind Pharm 2002, $28,1$.

[202] D.-H. Kim, D. C. Martin, Biomaterials 2006, 27, 3031.

[203] Y. Zhong, R. V. Bellamkonda, Brain Res. 2007, 1148, 15.

[204] X. Luo, C. Matranga, S. Tan, N. Alba, X. T. Cui, Biomaterials 2011, 32, 6316.

[205] R. Wadhwa, C. F. Lagenaur, X. T. Cui, J Control Release 2006, 110, 531.

[206] L. Spataro, J. Dilgen, S. Retterer, A. J. Spence, M. Isaacson, J. N. Turner, W. Shain, Exp. Neurol. 2005, 194, 289.

[207] E. J. Huang, L. F. Reichardt, Annu. Rev. Neurosci. 2001, 24, 677.

[208] S. M. Willerth, S. E. Sakiyama-Elbert, Advanced Drug Delivery Reviews 2007, 59, 325 .

[209] J. L. Ridet, S. K. Malhotra, A. Privat, F. H. Gage, Trends Neurosci. 1997, 20, 570.

[210] X. Cui, Biomaterials 2003, 24, 777.

[211] L. Kam, W. Shain, J. N. Turner, R. Bizios, Biomaterials 2002, 23, 511.

[212] S. Chen, N. Mantei, L. Dong, M. Schachner, J. Neurobiol. 1999, 38, 428.

[213] S. Khan, G. Newaz, J Biomed Mater Res A 2010, 93, 1209.

[214] S. S. Gill, N. K. Patel, G. R. Hotton, K. O'Sullivan, R. McCarter, M. Bunnage, D. J. Brooks, C. N. Svendsen, P. Heywood, Nature Medicine 2003, 9, 589.

[215] N. R. Herr, B. M. Kile, R. M. Carelli, R. M. Wightman, Anal. Chem. 2008, 80, 8635.

[216] J. Y. Sim, M. P. Haney, S. I. Park, J. G. McCall, J.-W. Jeong, Lab Chip 2017, 17, 1406.

[217] A. Pongrácz, Z. Fekete, G. Márton, Z. Bérces, I. Ulbert, P. Fürjes, Sensors and Actuators B: Chemical 2013, 189, 97.

[218] M. O. Heuschkel, L. Guérin, B. Buisson, D. Bertrand, P. Renaud, Sensors and Actuators B: Chemical 1998, 48, 356.

[219] H. Shin, H. J. Lee, U. Chae, H. Kim, J. Kim, N. Choi, J. Woo, Y. Cho, C. J. Lee, E.S. Yoon, I.-J. Cho, Lab Chip 2015, 15, 3730. 


\section{WILEY-VCH}

[220] K. Seidl, S. Spieth, S. Herwik, J. Steigert, R. Zengerle, O. Paul, P. Ruther, Journal of Micromechanics and Microengineering 2010, 20, 105006.

[221] A. Altuna, E. Bellistri, E. Cid, P. Aivar, B. Gal, J. Berganzo, G. Gabriel, A. Guimerá, R. Villa, L. J. Fernández, L. Menendez de la Prida, Lab Chip 2013, 13, 1422.

[222] S. Metz, A. Bertsch, D. Bertrand, P. Renaud, Biosens Bioelectron 2004, 19, 1309.

[223] D. Ziegler, T. Suzuki, S. Takeuchi, Journal of Microelectromechanical Systems 2006, 15, 1477.

[224] D. J. Laser, J. G. Santiago, Journal of Micromechanics and Microengineering 2004, 14, R35.

[225] A. Cobo, R. Sheybani, E. Meng, Adv Healthc Mater 2015, 4, 969.

[226] R. Sheybani, A. Cobo, E. Meng, Biomedical Microdevices 2015, 17, 74.

[227] N. M. Elman, H. L. Ho Duc, M. J. Cima, Biomedical Microdevices 2009, 11, 625.

[228] J. H. Prescott, S. Lipka, S. Baldwin, N. F. Sheppard, J. M. Maloney, J. Coppeta, B. Yomtov, M. A. Staples, J. T. Santini, Nat. Biotechnol. 2006, 24, 437.

[229] K. S. Soppimath, T. M. Aminabhavi, A. R. Kulkarni, W. E. Rudzinski, J Control Release 2001, 70, 1.

[230] I. I. Slowing, J. L. Vivero-Escoto, C.-W. Wu, V. S.-Y. Lin, Advanced Drug Delivery Reviews 2008, 60, 1278.

[231] P. Ghosh, G. Han, M. De, C. K. Kim, V. M. Rotello, Advanced Drug Delivery Reviews 2008, 60, 1307.

[232] E. J. Anglin, L. Cheng, W. R. Freeman, M. J. Sailor, Advanced Drug Delivery Reviews 2008, 60, 1266.

[233] A. T. Young, N. Cornwell, M. A. Daniele, Advanced Functional Materials 2017, 248, 1700239.

[234] E. Seker, Y. Berdichevsky, K. J. Staley, M. L. Yarmush, Adv Healthc Mater 2012, 1,172 .

[235] O. Polat, E. Seker, The Journal of Physical Chemistry C 2015, 119, 24812.

[236] S. D. Gittard, B. E. Pierson, C. M. Ha, C.-A. M. Wu, R. J. Narayan, D. B. Robinson, Biotechnology Journal 2010, 5, 192.

[237] M. R. Abidian, D. C. Martin, Advanced Functional Materials 2009, 19, 573.

[238] M. R. Abidian, D.-H. Kim, D. C. Martin, Adv. Mater. Weinheim 2006, 18, 405.

[239] M. K. Gheith, T. C. Pappas, A. V. Liopo, V. A. Sinani, B. S. Shim, M. Motamedi, J. P. Wicksted, N. A. Kotov, Advanced Materials 2006, 18, 2975.

[240] E. S. Boyden, F. Zhang, E. Bamberg, G. Nagel, K. Deisseroth, Nat. Neurosci. 2005, $8,1263$.

[241] F. Zhang, L.-P. Wang, M. Brauner, J. F. Liewald, K. Kay, N. Watzke, P. G. Wood,

E. Bamberg, G. Nagel, A. Gottschalk, K. Deisseroth, Nature 2007, 446, 633.

[242] X. Han, E. S. Boyden, PLoS ONE 2007, 2, e299.

[243] F. Zhang, M. Prigge, F. Beyrière, S. P. Tsunoda, J. Mattis, O. Yizhar, P. Hegemann, K. Deisseroth, Nat. Neurosci. 2008, 11, 631.

[244] J. Y. Lin, P. M. Knutsen, A. Muller, D. Kleinfeld, R. Y. Tsien, Nat. Neurosci. 2013, 16, 1499.

[245] J. Wietek, J. S. Wiegert, N. Adeishvili, F. Schneider, H. Watanabe, S. P. Tsunoda, A. Vogt, M. Elstner, T. G. Oertner, P. Hegemann, Science 2014, 344, 409.

[246] B. R. Arenkiel, J. Peca, I. G. Davison, C. Feliciano, K. Deisseroth, G. J. Augustine, M. D. Ehlers, G. Feng, Neuron 2007, 54, 205.

[247] A. V. Kravitz, B. S. Freeze, P. R. L. Parker, K. Kay, M. T. Thwin, K. Deisseroth, A.

C. Kreitzer, Nature 2010, 466, 622.

[248] K. M. Tye, K. Deisseroth, Nat. Rev. Neurosci. 2012, 13, 251.

[249] B. K. Andrasfalvy, B. V. Zemelman, J. Tang, A. Vaziri, Proc. Natl. Acad. Sci. 


\section{WILEY-VCH}

U.S.A. 2010, 107, 11981.

[250] A. M. Packer, D. S. Peterka, J. J. Hirtz, R. Prakash, K. Deisseroth, R. Yuste, Nature Methods 2012, 9, 1202.

[251] E. Papagiakoumou, F. Anselmi, A. Bègue, V. de Sars, J. Glückstad, E. Y. Isacoff, V. Emiliani, Nature Methods 2010, 7, 848.

[252] M. Dal Maschio, J. C. Donovan, T. O. Helmbrecht, H. Baier, Neuron 2017, 94, 774.

[253] A. Vaziri, V. Emiliani, Curr. Opin. Neurobiol. 2012, 22, 128.

[254] D. R. Sparta, A. M. Stamatakis, J. L. Phillips, N. Hovelsø, R. van Zessen, G. D. Stuber, Nature Protocols 2011, 7, 12.

[255] V. Gradinaru, M. Mogri, K. R. Thompson, J. M. Henderson, K. Deisseroth, Science 2009, 324, 354.

[256] S. Jego, S. D. Glasgow, C. G. Herrera, M. Ekstrand, S. J. Reed, R. Boyce, J. Friedman, D. Burdakov, A. R. Adamantidis, Nat. Neurosci. 2013, 16, 1637.

[257] A. V. Kravitz, A. C. Kreitzer, Curr. Opin. Neurobiol. 2011, 21, 433.

[258] C. T. Wentz, J. G. Bernstein, P. Monahan, A. Guerra, A. Rodriguez, E. S. Boyden, J Neural Eng 2011, 8, 046021.

[259] D. Huber, L. Petreanu, N. Ghitani, S. Ranade, T. Hromádka, Z. Mainen, K. Svoboda, Nature 2008, 451, 61.

[260] D.-W. Park, A. A. Schendel, S. Mikael, S. K. Brodnick, T. J. Richner, J. P. Ness, M. R. Hayat, F. Atry, S. T. Frye, R. Pashaie, S. Thongpang, Z. Ma, J. C. Williams, Nature Communications 2014, 5, 5258.

[261] P. Ledochowitsch, E. Olivero, T. Blanche, M. M. Maharbiz, IEEE, 2011; pp. 29372940.

[262] F. Wu, E. Stark, M. Im, I.-J. Cho, E.-S. Yoon, G. Buzsáki, K. D. Wise, E. Yoon, J Neural Eng 2013, 10, 056012.

[263] A. N. Zorzos, E. S. Boyden, C. G. Fonstad, Opt Lett 2010, 35, 4133.

[264] Y. Son, H. J. Lee, D. Kim, Y. K. Kim, E.-S. Yoon, J. Y. Kang, N. Choi, T. G. Kim, I.-J. Cho, IEEE, 2014; pp. 853-856.

[265] I.-J. Cho, H. W. Baac, E. Yoon, IEEE; pp. 995-998.

[266] K. Y. Kwon, H.-M. Lee, M. Ghovanloo, A. Weber, W. Li, IEEE, 2014; pp. 813816.

[267] J. Missinne, S. Kalathimekkad, B. Van Hoe, E. Bosman, J. Vanfleteren, G. Van Steenberge, Opt Express 2014, 22, 4168.

[268] N. McAlinden, E. Gu, M. D. Dawson, S. Sakata, K. Mathieson, Front Neural Circuits 2015, 9, 420.

[269] T. I. Kim, J. G. McCall, Y. H. Jung, X. Huang, E. R. Siuda, Y. Li, J. Song, Y. M. Song, H. A. Pao, R. H. Kim, C. Lu, S. D. Lee, I. S. Song, G. Shin, R. Al-Hasani, S. Kim, M. P. Tan, Y. Huang, F. G. Omenetto, J. A. Rogers, M. R. Bruchas, Science 2013, 340, 211.

[270] H. Cao, L. Gu, S. K. Mohanty, J. C. Chiao, IEEE Transactions on Biomedical Engineering 2013, 60, 225.

[271] J. G. McCall, T.-I. Kim, G. Shin, X. Huang, Y. H. Jung, R. Al-Hasani, F. G. Omenetto, M. R. Bruchas, J. A. Rogers, Nature Protocols 2013, 8, 2413.

[272] N. McAlinden, D. Massoubre, E. Richardson, E. Gu, S. Sakata, M. D. Dawson, K. Mathieson, Opt Lett 2013, 38, 992.

[273] T.-I. Kim, Y. H. Jung, J. Song, D. Kim, Y. Li, H.-S. Kim, I.-S. Song, J. J. Wierer, H. A. Pao, Y. Huang, J. A. Rogers, Small 2012, 8, 1643.

[274] B. Fan, W. Li, Lab Chip 2015, 15, 3838.

[275] X. Wu, Y. Zhang, K. Takle, O. Bilsel, Z. Li, H. Lee, Z. Zhang, D. Li, W. Fan, C. Duan, E. M. Chan, C. Lois, Y. Xiang, G. Han, ACS Nano 2016, 10, 1060.

[276] Y. Zhang, L. Huang, Z. Li, G. Ma, Y. Zhou, G. Han, ACS Nano 2016, 10, 3881. 


\section{WILEY-VCH}

[277] S. Shah, J.-J. Liu, N. Pasquale, J. Lai, H. McGowan, Z. P. Pang, K.-B. Lee, Nanoscale 2015, 7, 16571.

[278] A. Bansal, H. Liu, M. K. G. Jayakumar, S. Andersson-Engels, Y. Zhang, Small 2016, 12, 1732.

[279] T. C. Pappas, W. M. S. Wickramanyake, E. Jan, M. Motamedi, M. Brodwick, N. A. Kotov, Nano Lett. 2007, 7, 513.

[280] J. L. Carvalho-de-Souza, J. S. Treger, B. Dang, S. B. H. Kent, D. R. Pepperberg, F. Bezanilla, Neuron 2015, 86, 207.

[281] S. Yoo, S. Hong, Y. Choi, J.-H. Park, Y. Nam, ACS Nano 2014, 8, 8040.

[282] K. Eom, J. Kim, J. M. Choi, T. Kang, J. W. Chang, K. M. Byun, S. B. Jun, S. J. Kim, Small 2014, 10, 3853.

[283] Y. Pan, S. Neuss, A. Leifert, M. Fischler, F. Wen, U. Simon, G. Schmid, W. Brandau, W. Jahnen-Dechent, Small 2007, 3, 1941.

[284] S.-M. Chuang, Y.-H. Lee, R.-Y. Liang, G.-D. Roam, Z.-M. Zeng, H.-F. Tu, S.-K. Wang, P. J. Chueh, Biochim. Biophys. Acta 2013, 1830, 4960.

[285] A. Q. Tran, C. Kaulen, U. Simon, A. Offenhäusser, D. Mayer, Biomater. Sci. 2017, $5,1051$.

[286] S. Ding, Y. Liu, Y. Li, Z. Liu, S. Sohn, F. J. Walker, J. Schroers, Nature Materials 2014, 13, 494.

[287] A. L. Hook, D. G. Anderson, R. Langer, P. Williams, M. C. Davies, M. R. Alexander, Biomaterials 2010, 31, 187.

[288] R. Potyrailo, K. Rajan, K. Stoewe, I. Takeuchi, B. Chisholm, H. Lam, ACS Combinatorial Science 2011, 13, 579.

[289] A. J. Vegas, O. Veiseh, J. C. Doloff, M. Ma, H. H. Tam, K. Bratlie, J. Li, A. R. Bader, E. Langan, K. Olejnik, P. Fenton, J. W. Kang, J. Hollister-Locke, M. A. Bochenek, A. Chiu, S. Siebert, K. Tang, S. Jhunjhunwala, S. Aresta-Dasilva, N. Dholakia, R. Thakrar, T. Vietti, M. Chen, J. Cohen, K. Siniakowicz, M. Qi, J. McGarrigle, S. Lyle, D. M. Harlan, D. L. Greiner, J. Oberholzer, G. C. Weir, R. Langer, D. G. Anderson, Nat. Biotechnol. 2016, 34, 345.

[290] A. L. Hook, C.-Y. Chang, J. Yang, J. Luckett, A. Cockayne, S. Atkinson, Y. Mei, R. Bayston, D. J. Irvine, R. Langer, D. G. Anderson, P. Williams, M. C. Davies, M. R. Alexander, Nat. Biotechnol. 2012, 30, 868.

[291] G. A. Higuera, J. A. A. Hendriks, J. van Dalum, L. Wu, R. Schotel, L. MoreiraTeixeira, M. van den Doel, J. C. H. Leijten, J. Riesle, M. Karperien, C. A. van Blitterswijk, L. Moroni, Integr Biol (Camb) 2013, 5, 889.

[292] L. R. Giam, M. D. Massich, L. Hao, L. Shin Wong, C. C. Mader, C. A. Mirkin, Proc. Natl. Acad. Sci. U.S.A. 2012, 109, 4377.

[293] V. Z. Beachley, M. T. Wolf, K. Sadtler, S. S. Manda, H. Jacobs, M. R. Blatchley, J. S. Bader, A. Pandey, D. Pardoll, J. H. Elisseeff, Nature Methods 2015, 12, 1197.

[294] A. Solanki, S. Shah, K. A. Memoli, S. Y. Park, S. Hong, K.-B. Lee, Small 2010, 6, 2509.

[295] C. A. R. Chapman, L. Wang, J. Biener, E. Seker, M. M. Biener, M. J. Matthews, Nanoscale 2015, 8, 785 .

[296] C. A. R. Chapman, X. Zhu, H. Chen, A. A. Yanik, P. J. Lein, E. Seker, Sci Rep 2017, 7, 427.

[297] R. E. Fischell, D. R. Fischell, Integrated system for EEG monitoring and electrical stimulation with a multiplicity of electrodes.

[298] R. H. Olsson, D. L. Buhl, A. M. Sirota, G. Buzsáki, K. D. Wise, IEEE Transactions on Biomedical Engineering 2005, 52, 1303.

[299] M. Vomero, E. Castagnola, F. Ciarpella, E. Maggiolini, N. Goshi, E. Zucchini, S. Carli, L. Fadiga, S. Kassegne, D. Ricci, Sci Rep 2017, 7, 40332. 


\section{WILEY-VCH}

[300] J. Isaksson, P. Kjäll, D. Nilsson, N. D. Robinson, M. Berggren, A. RichterDahlfors, Nature Materials 2007, 6, 673.

[301] J. Chen, K. D. Wise, J. F. Hetke, S. C. Bledsoe, IEEE Transactions on Biomedical Engineering 1997, 44, 760.

[302] D. T. Simon, S. Kurup, K. C. Larsson, R. Hori, K. Tybrandt, M. Goiny, E. W. H. Jager, M. Berggren, B. Canlon, A. Richter-Dahlfors, Nature Materials 2009, 8, 742.

[303] D. P. Papageorgiou, S. E. Shore, S. C. Bledsoe, K. D. Wise, Journal of Microelectromechanical Systems 2006, 15, 1025.

[304] A. Canales, X. Jia, U. P. Froriep, R. A. Koppes, C. M. Tringides, J. Selvidge, C. Lu, C. Hou, L. Wei, Y. Fink, P. Anikeeva, Nat. Biotechnol. 2015, 33, 277.

[305] I. Ozden, J. Wang, Y. Lu, T. May, J. Lee, W. Goo, D. J. O'Shea, P. Kalanithi, I. Diester, M. Diagne, K. Deisseroth, K. V. Shenoy, A. V. Nurmikko, J. Neurosci. Methods 2013, 219, 142.

[306] K. Tamura, Y. Ohashi, T. Tsubota, D. Takeuchi, T. Hirabayashi, M. Yaguchi, M. Matsuyama, T. Sekine, Y. Miyashita, J. Neurosci. Methods 2012, 211, 49.

[307] D. Kuzum, H. Takano, E. Shim, J. C. Reed, H. Juul, A. G. Richardson, J. de Vries, H. Bink, M. A. Dichter, T. H. Lucas, D. A. Coulter, E. Cubukcu, B. Litt, Nature Communications 2014, 5, 5259.

[308] S. Chen, W. Pei, Q. Gui, Y. Chen, S. Zhao, H. Wang, H. Chen, J Neural Eng 2013, 10, 046020.

[309] V. Busskamp, S. Picaud, J. A. Sahel, B. Roska, Gene Ther. 2012, 19, 169.

[310] S. Dufour, P. Dufour, O. Chever, R. Vallée, F. Amzica, J. Neurosci. Methods 2011, 194, 206.

[311] A. M. Belle, C. Owesson-White, N. R. Herr, R. M. Carelli, R. M. Wightman, ACS Chem Neurosci 2013, 4, 761.

[312] J.-W. Jeong, J. G. McCall, G. Shin, Y. Zhang, R. Al-Hasani, M. Kim, S. Li, J. Y. Sim, K.-I. Jang, Y. Shi, D. Y. Hong, Y. Liu, G. P. Schmitz, L. Xia, Z. He, P. Gamble, W. Z. Ray, Y. Huang, M. R. Bruchas, J. A. Rogers, Cell 2015, 162, 662.

[313] J. P. Rickgauer, K. Deisseroth, D. W. Tank, Nat. Neurosci. 2014, 17, 1816.

[314] F. Lavoie-Cardinal, C. Salesse, É. Bergeron, M. Meunier, P. De Koninck, Sci Rep 2016, 6, 389.

[315] J. Zhang, F. Laiwalla, J. A. Kim, H. Urabe, R. Van Wagenen, Y.-K. Song, B. W. Connors, F. Zhang, K. Deisseroth, A. V. Nurmikko, J Neural Eng 2009, 6, 055007.

[316] T. D. Y. Kozai, N. B. Langhals, P. R. Patel, X. Deng, H. Zhang, K. L. Smith, J. Lahann, N. A. Kotov, D. R. Kipke, Nature Materials 2012, 11, 1065.

[317] E. Seker, M. Reed, M. Utz, M. R. Begley, Applied Physics Letters 2008, 92, 154101.

[318] M. Vomero, P. van Niekerk, V. Nguyen, N. Gong, M. Hirabayashi, A. Cinopri, K. Logan, A. Moghadasi, P. Varma, S. Kassegne, Journal of Micromechanics and Microengineering 2016, 26, 025018.

[319] D. Khodagholy, J. N. Gelinas, T. Thesen, W. Doyle, O. Devinsky, G. G. Malliaras, G. Buzsáki, NeuroGrid: recording action potentials from the surface of the brain.; 2015; Vol. 18, pp. 310-315.

[320] A. Blau, A. Murr, S. Wolff, E. Sernagor, P. Medini, G. Iurilli, C. Ziegler, F. Benfenati, Biomaterials 2011, 32, 1778.

[321] I. R. Minev, P. Musienko, A. Hirsch, Q. Barraud, N. Wenger, E. M. Moraud, J. Gandar, M. Capogrosso, T. Milekovic, L. Asboth, R. F. Torres, N. Vachicouras, Q. Liu, N. Pavlova, S. Duis, A. Larmagnac, J. Vörös, S. Micera, Z. Suo, G. Courtine, S. P. Lacour, Science 2015, 347, 159.

[322] D. Seo, R. M. Neely, K. Shen, U. Singhal, E. Alon, J. M. Rabaey, J. M. Carmena, M. M. Maharbiz, Neuron 2016, 91, 529. 


\section{WILEY-VCH}

[323] Y. Brudno, E. A. Silva, C. J. Kearney, S. A. Lewin, A. Miller, K. D. Martinick, M. Aizenberg, D. J. Mooney, Proc. Natl. Acad. Sci. U.S.A. 2014, 111, 12722.

[324] E. O. Gabrielsson, P. Janson, K. Tybrandt, D. T. Simon, M. Berggren, Advanced Materials 2014, 26, 5143.

[325] S. M. Wellman, J. R. Eles, K. A. Ludwig, J. P. Seymour, N. J. Michelson, W. E. McFadden, A. L. Vazquez, T. D. Y. Kozai, Advanced Functional Materials 2017, 484, 1701269.

[326] K. Bowsher, E. F. Civillico, J. Coburn, J. Collinger, J. L. Contreras-Vidal, T. Denison, J. Donoghue, J. French, N. Getzoff, L. R. Hochberg, M. Hoffmann, J. Judy, N. Kleitman, G. Knaack, V. Krauthamer, K. Ludwig, M. Moynahan, J. J. Pancrazio, P. H. Peckham, C. Pena, V. Pinto, T. Ryan, D. Saha, H. Scharen, S. Shermer, K. Skodacek, P. Takmakov, D. Tyler, S. Vasudevan, K. Wachrathit, D. Weber, C. G. Welle, M. Ye, IOP Publishing, 2016; Vol. 13, p. 023001.

[327] X. I. A. G. B. R. J. H.-F. I. M.-F. M. T. M. P. G. M. V. S.-V. Gemma Gabriel, R. Villa, In Physical and Chemical Properties of Carbon Nanotubes; InTech, 2013.

[328] I. Yoon, K. Hamaguchi, I. V. Borzenets, G. Finkelstein, R. Mooney, B. R. Donald, PLoS ONE 2013, 8, e65715.

[329] A. G. Zestos, C. Yang, C. B. Jacobs, D. Hensley, B. J. Venton, Analyst 2015, 140, 7283.

[330] N. Vasylieva, S. Marinesco, D. Barbier, A. Sabac, Biosens Bioelectron 2015, 72, 148.

[331] D. Chen, W. Du, Y. Liu, W. Liu, A. Kuznetsov, F. E. Mendez, L. H. Philipson, R. F. Ismagilov, Proc. Natl. Acad. Sci. U.S.A. 2008, 105, 16843.

[332] Y. Gong, C. Huang, J. Z. Li, B. F. Grewe, Y. Zhang, S. Eismann, M. J. Schnitzer, Science 2015, 350, 1361.

[333] F. Zhang, V. Gradinaru, A. R. Adamantidis, R. Durand, R. D. Airan, L. de Lecea, K. Deisseroth, Nature Protocols 2010, 5, 439.

[334] K. Y. Kwon, B. Sirowatka, A. Weber, W. Li, IEEE Transactions on Biomedical Circuits and Systems 2013, 7, 593. 


\section{WILEY-VCH}

\section{FIGURES \& CAPTIONS}

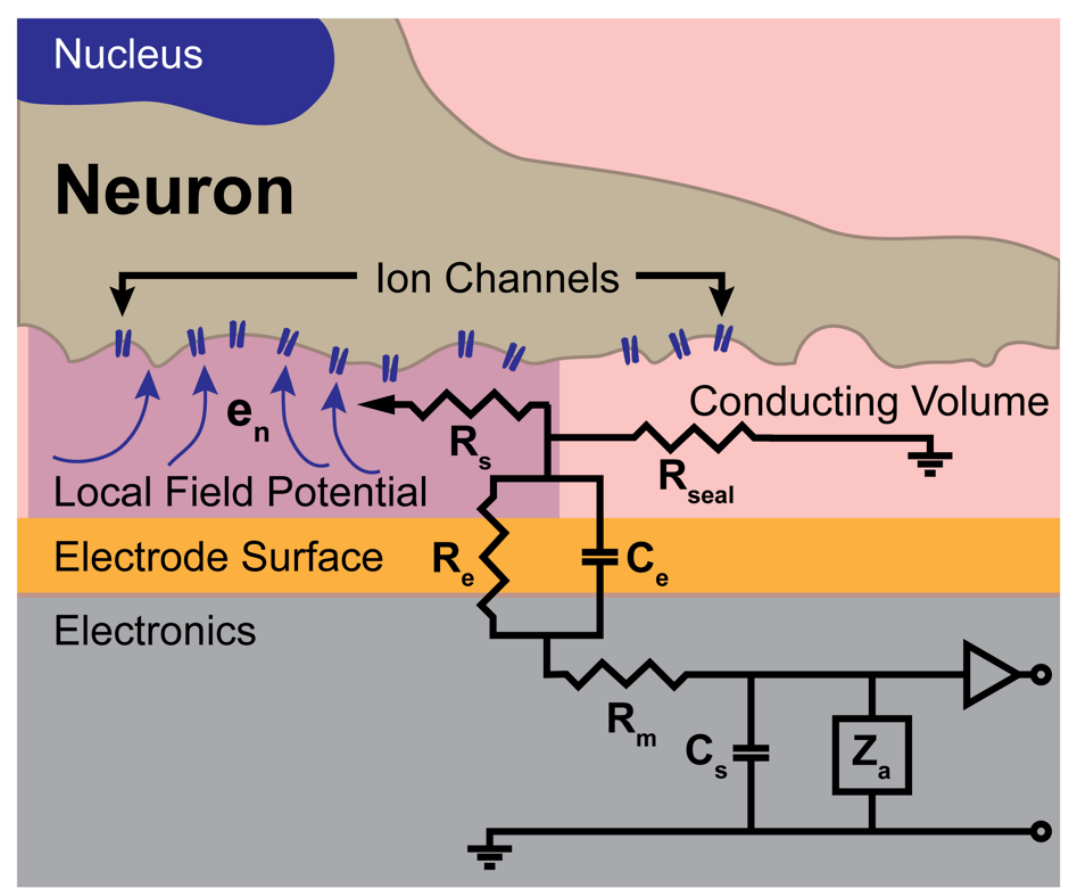

Figure 1. Equivalent circuit model of the electrical recording interface. The neural electrical interface transduces the local field potential $\left(e_{n}\right)$, which is a combination of signals from a volume of neurons. Physiological buffer resistance $\left(\mathrm{R}_{\mathrm{s}}\right)$, metal trace resistance $\left(\mathrm{R}_{\mathrm{m}}\right)$, shunt capacitance $\left(C_{s}\right)$, and amplifier input impedance $\left(Z_{a}\right)$ are typically not limiting factors in monitoring performance. The seal resistance $\left(R_{\text {seal }}\right)$ and electrode impedance $\left(Z_{e}-\right.$ determined by $\mathrm{R}_{\mathrm{e}}$ and $\mathrm{C}_{\mathrm{e}}$ ) are the dominant parameters that determine the monitoring performance at a neural-electrical interface. 


\section{WILEY-VCH}

A

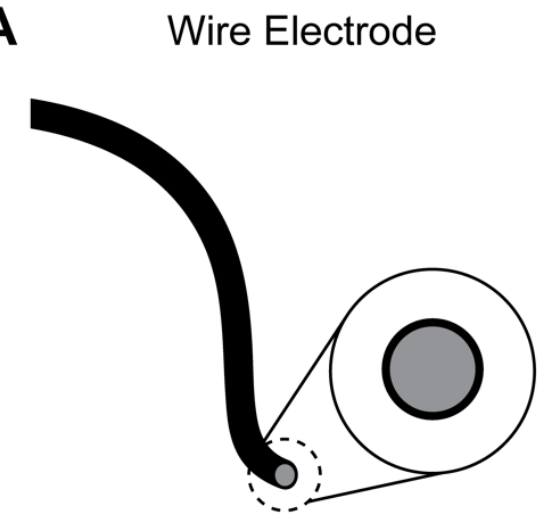

C Michigan Style Array

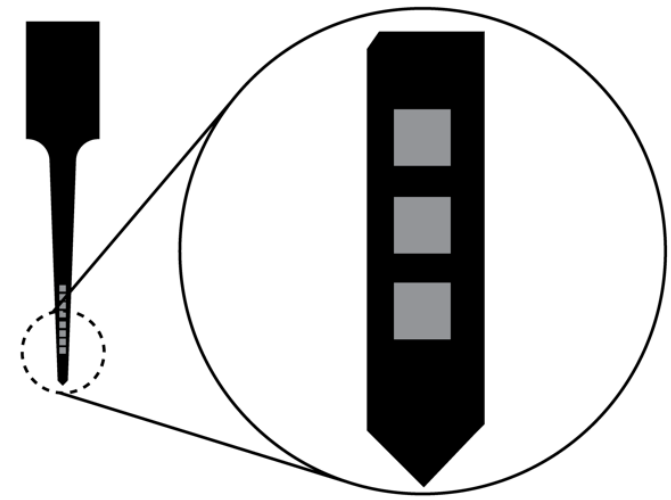

B

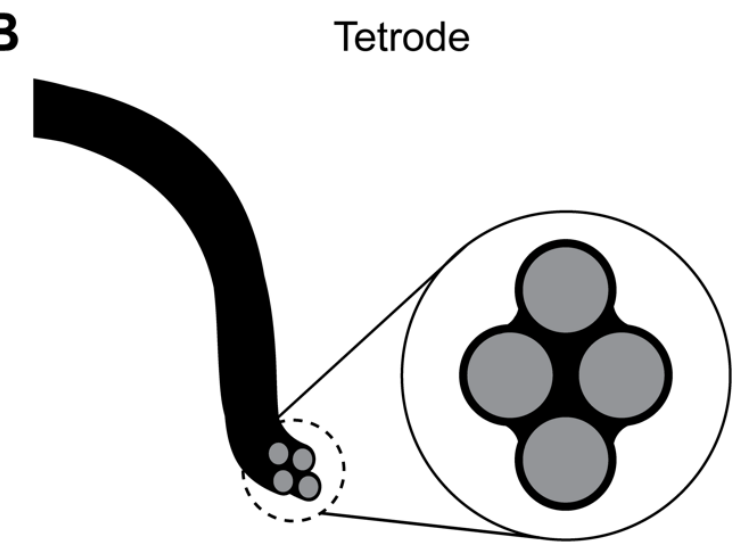

D

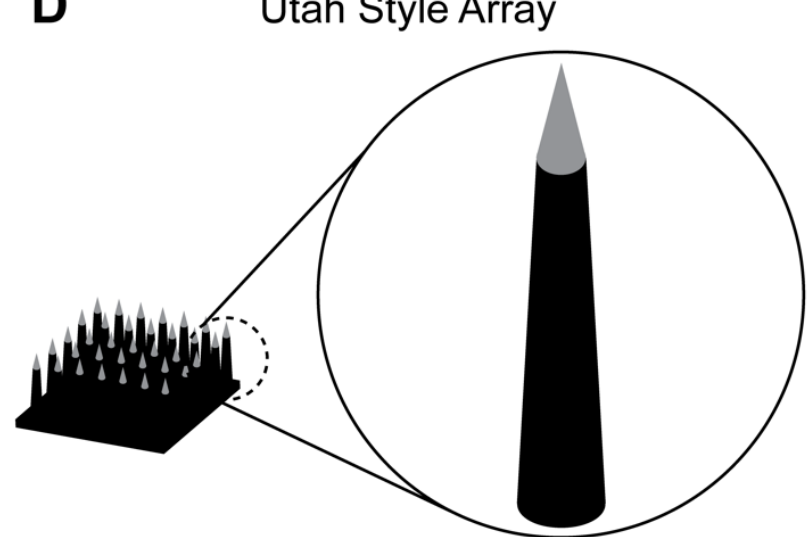

Figure 2. Schematic illustrating common electrode geometries. (A) Wire electrode consisting of a conducting wire (grey) and insulating sheath (black). (B) Tetrode consisting of four conjoined wire electrodes. (C) Michigan style array consisting of electrodes (grey) on a single plane of a shank (black). (D) Utah style array consisting of insulated pillars (black) with conducting electrode tips (grey). 


\section{WILEY-VCH}

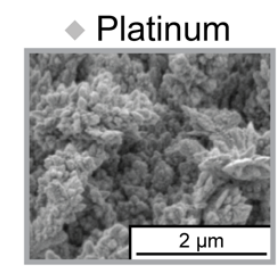

- CNTS

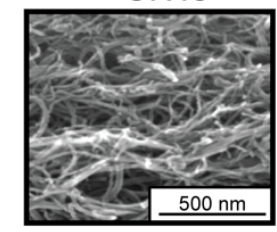

$\checkmark$ Titanium Nitride
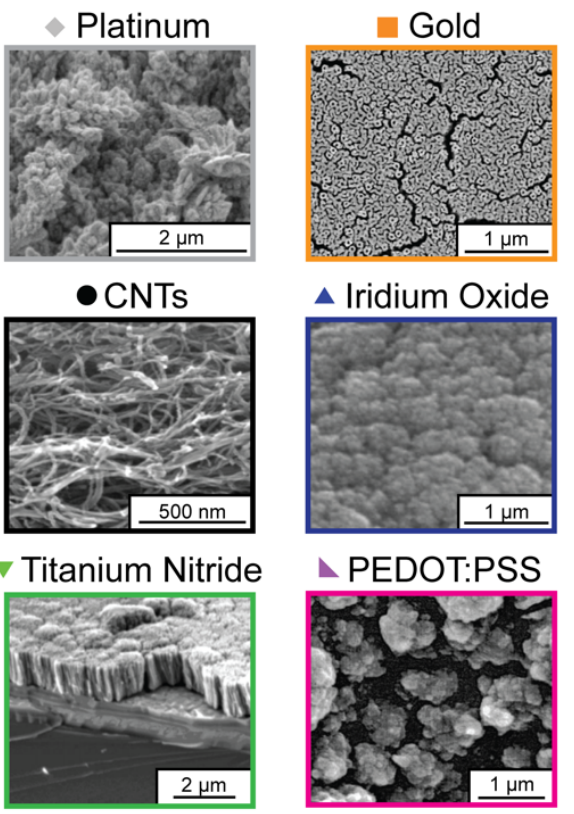

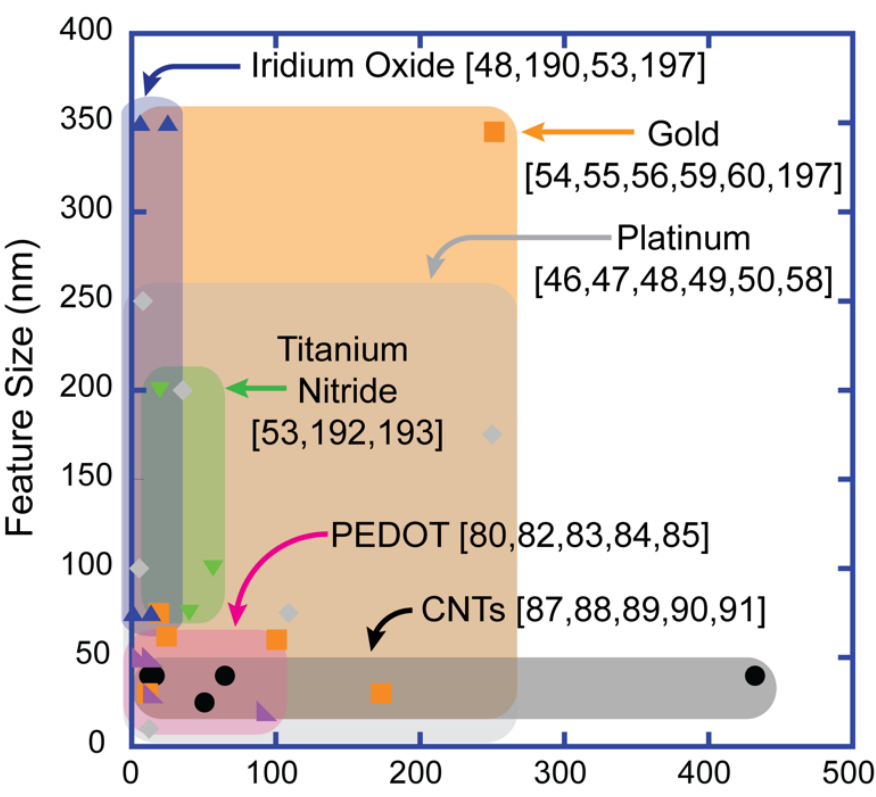

Normalized Impedance $\left(\mathrm{Z}_{\mathrm{e}}\right)$ at $1 \mathrm{kHz}\left(\mathrm{k} \Omega^{*} \mathrm{~mm}^{2}\right)$
B

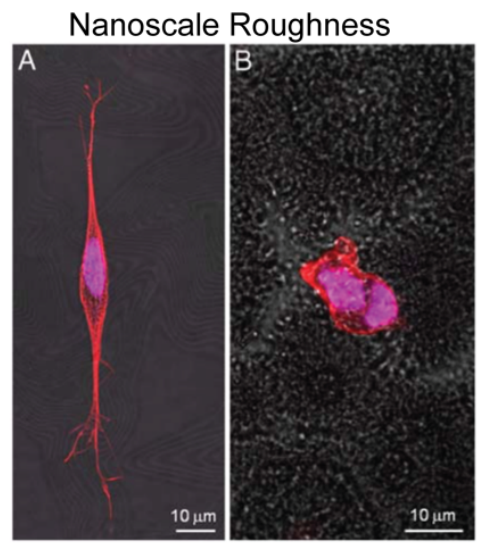

Controlling Cell Coupling through Nanoscale Features
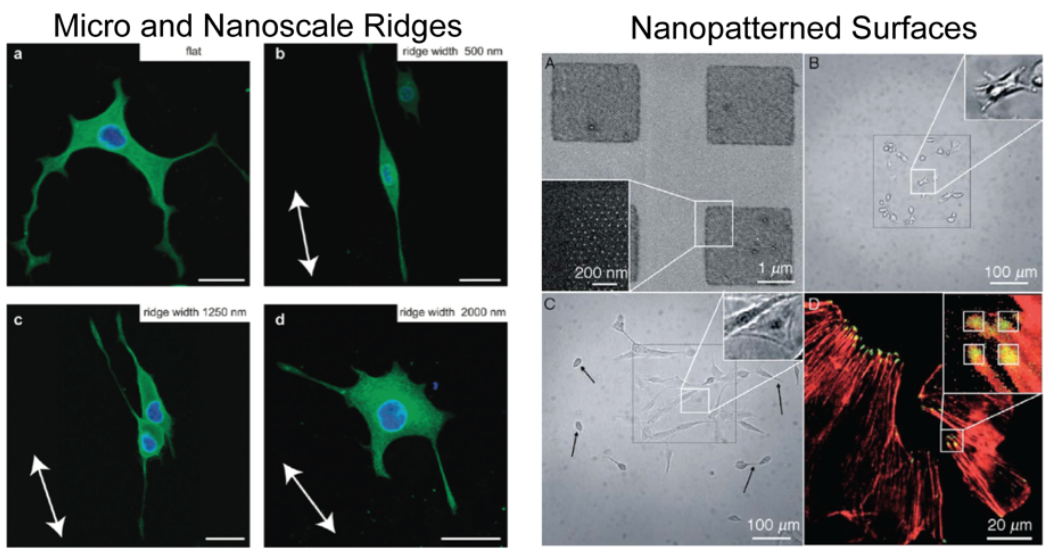

Figure 3. Examples of techniques to enhance electrical monitoring of neural activity. (A) (Left) Representative scanning electron micrographs of nanostructured materials (Platinum ${ }^{[327]}$ Reproduced per Creative Commons License, Gold ${ }^{[54]}$ Reproduced with permission. Copyright 2015, WILEY-VCH Verlag GmbH \& Co. KGaA. Carbon Nanotubes ${ }^{[328]}$ Reproduced per Creative Commons License, Iridium Oxide ${ }^{[52]}$ Reproduced with permission. Copyright 2004, IEEE. Titanium Nitride ${ }^{[53]}$ Reproduced with permission. Copyright 2002, IEEE. PEDOT:PSS ${ }^{[2]}$ Reproduced with permission. Copyright 2003, Elsevier B.V.). (Right) Representative graph illustrating the relationship between nanoscale feature size (estimated from scanning electron micrographs) and electrode impedances normalized to geometric electrode area reported in literature for each material. PEDOT:PSS, CNTs, titanium nitride, and iridium oxide all stand out with markedly lower electrode impedances compared to gold and platinum. However, gold and platinum appear to be fabricated in a significantly larger range of nanostructure. (B) Images demonstrating the use of nanostructure to control cellular coupling to underlying substrate and alignment. (Left) The effect of nanoscale roughness on the SHSY5Y human neuroblastoma cell line ${ }^{[69]}$ (Reproduced with permission. National Academy of Science). (Middle) The effect of micro- and nanoscale ridges on PC12 rat adrenal medulla cell line ${ }^{[70]}$ (Reproduced with permission. Copyright 2001, American Chemical Society). (Right) 


\section{WILEY-VCH}

The effect of nanopatterned surfaces on MC3T3 osteoblast focal adhesion formation ${ }^{[73]}$. Reproduced with permission. Copyright 2007, Elsevier. 


\section{WILEY-VCH}
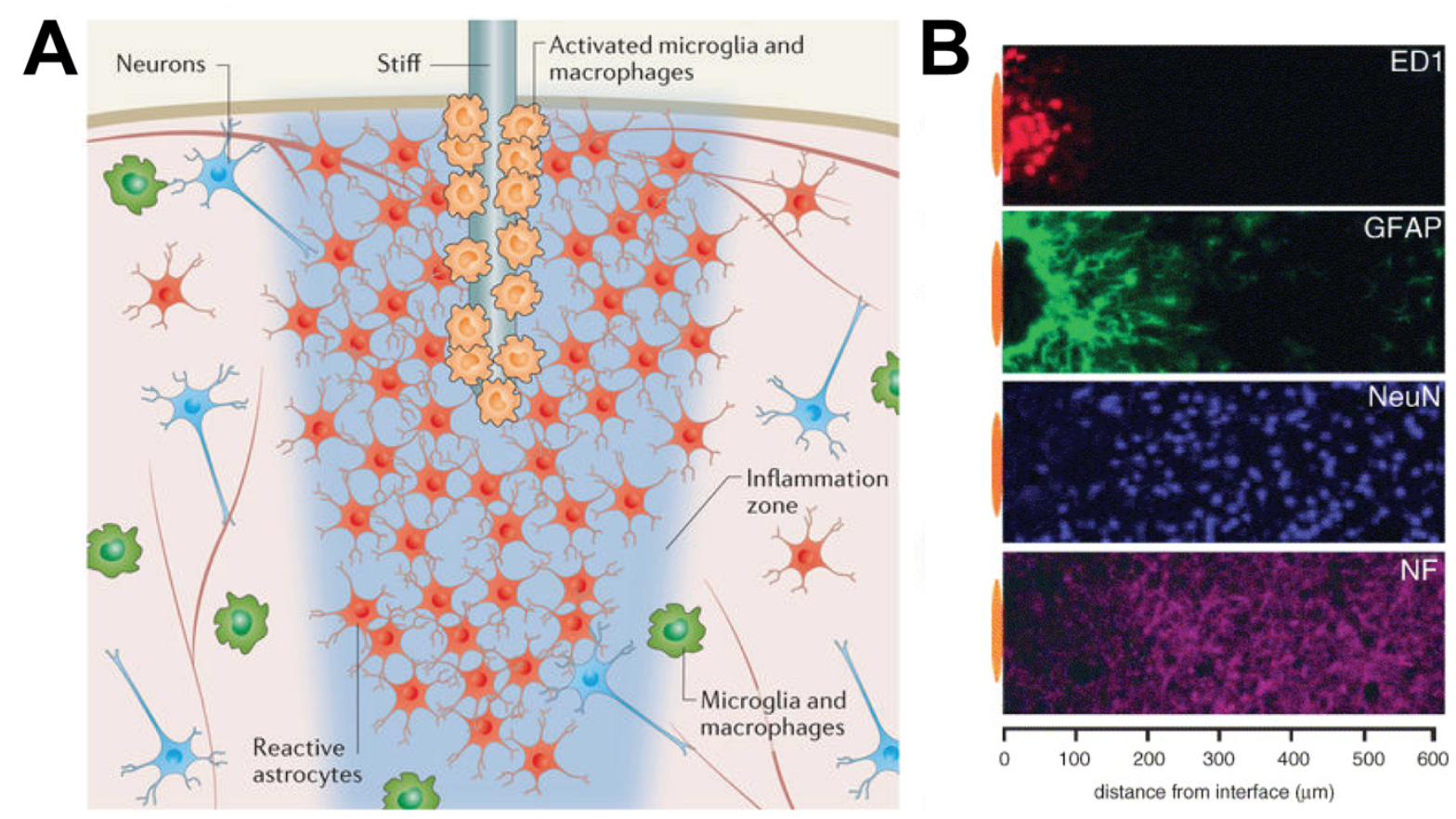

Figure 4. Schematic illustrating the adverse tissue response to a stiff intracranial implant. (A) Initial penetration and subsequent micro-motion around the implant leads to increased inflammatory response around the implant. This response induces astrocytes to a reactive phenotype that leads to scarring around the implant surface ${ }^{[78]}$. Copyright 2016, Nature Publishing Group. (B) Visualization of the scar formation around an implanted electrode site. Activated microglia (ED1 - red) cluster around the electrode surface leading to encapsulation by astrocytes (GFAP - green) which leads to the distancing of neuron cell bodies (NeuN - blue) and neuronal processes (NF - purple) from the surface of the electrode. This scarring both introduces an ionically-insulating layer to the surface and distances neurons from the electrode, which ultimately reduces the ability to effectively monitor signals from the surrounding cells ${ }^{[44]}$. Copyright 2005, Elsevier. 


\section{WILEY-VCH}

A
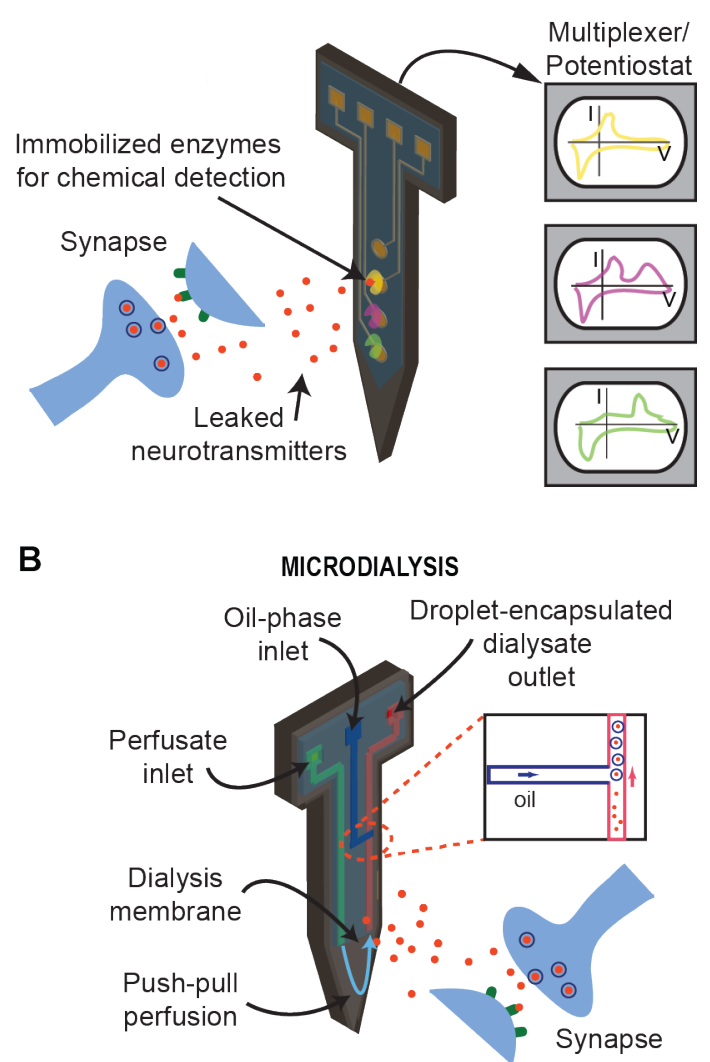

Figure 5. Schematic illustration of chemical interfaces for monitoring neural activity. (A) In electrochemical-detection, leaked neurotransmitters from the synaptic cleft and metabolites in the interstitial space are oxidized or reduced by applying an electrical potential between a working electrode (bare or coated with enzymes that are specific to the analyte of interest) and reference electrode. If the analyte is electroactive, at a molecule-dependent potential, an electrochemical current is produced due to the oxidation event. This current reveals the number of molecules detected, while the corresponding potential serves as an identifier for the analyte. (B) In microdialysis, the interstitial space is sampled through the diffusion of analytes through a semipermeable dialysis membrane, where the analytes are encapsulated in micro-emulsions to prevent their diffusive-dilution and detected downstream via various modalities (e.g., electrochemical, fluorescence). 


\section{WILEY-VCH}

A
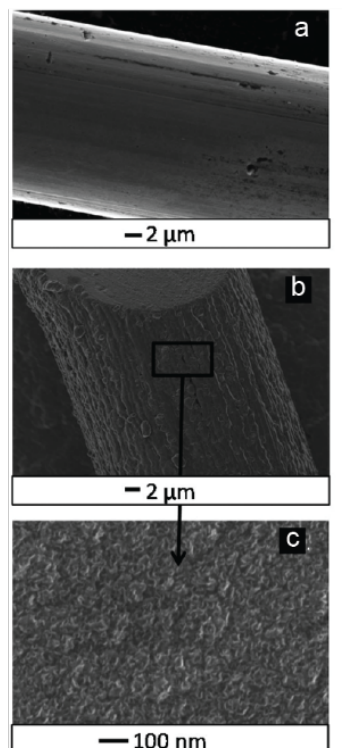

$-100 \mathrm{~nm}$

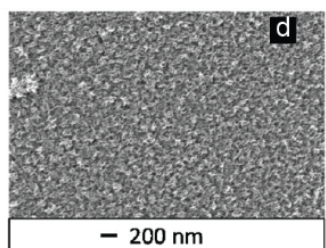

$-200 \mathrm{~nm}$
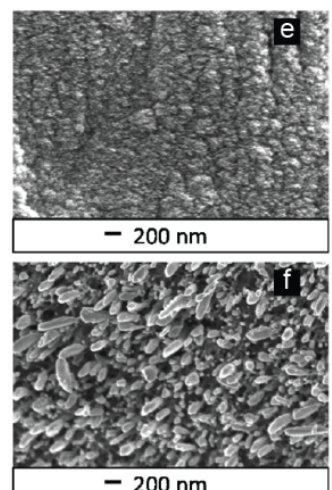

$-200 \mathrm{~nm}$
B
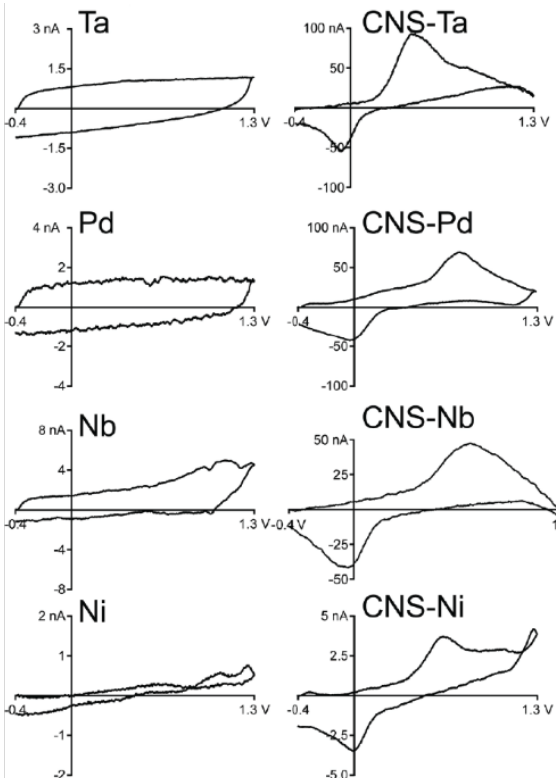

${ }_{100 n A}$ CNS-Pd
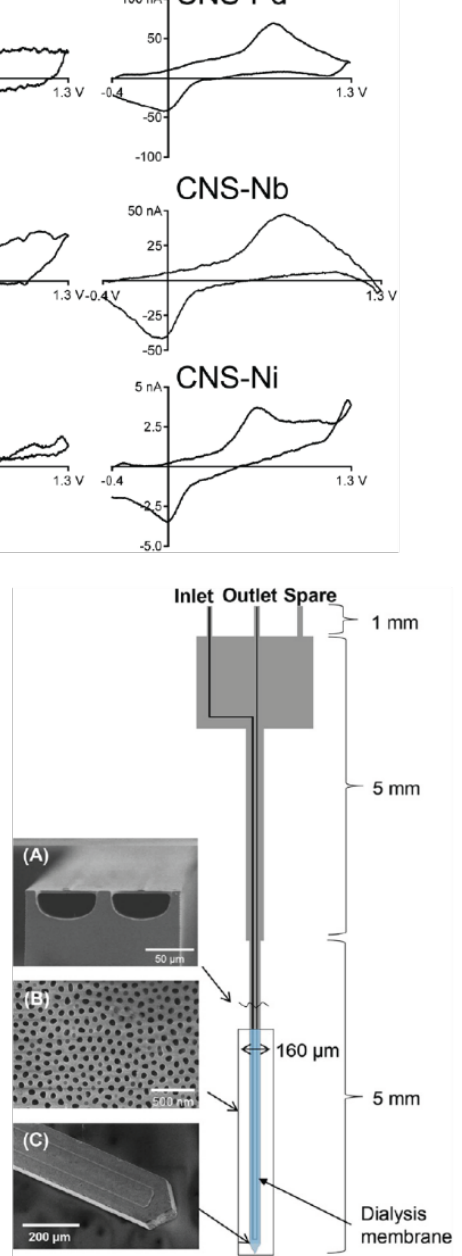

C

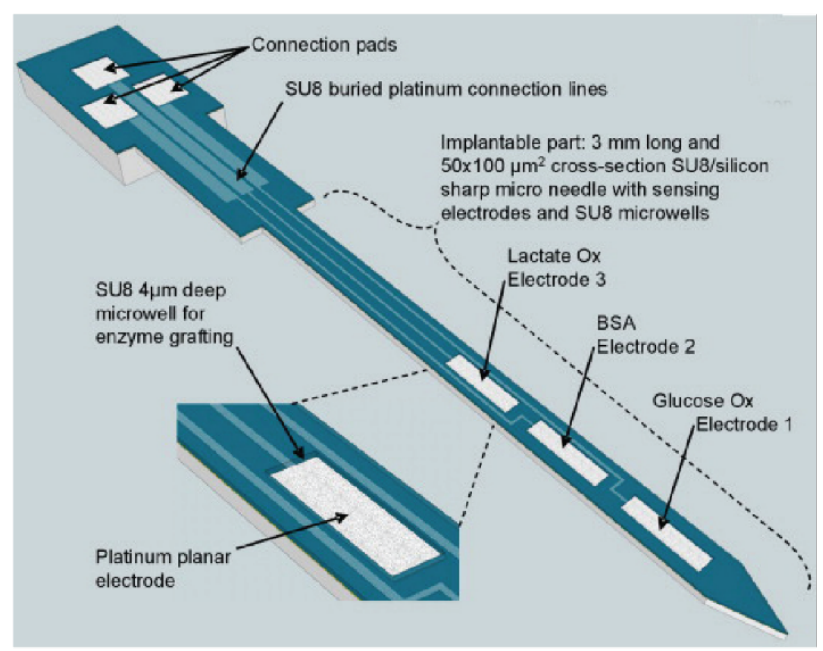

D
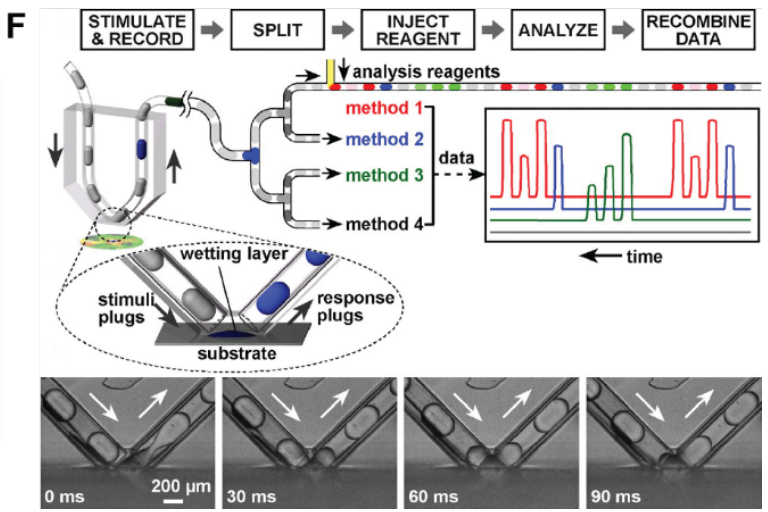

E

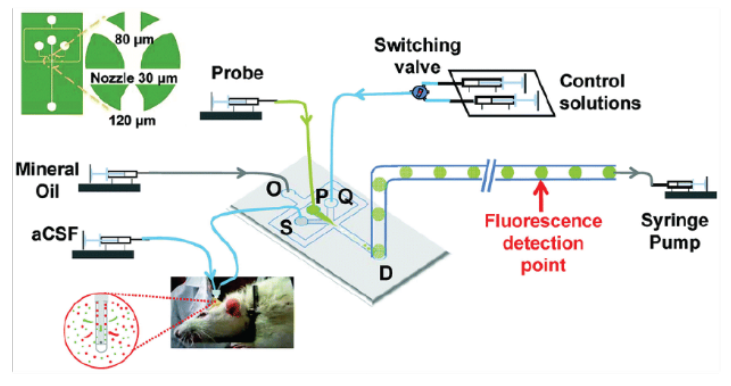

Figure 6. Examples of chemical monitoring technologies. (A) Scanning electron microscope images of insulated fibers of different metals coated with carbon nanospikes (CNS) via plasmaenhanced chemical vapor deposition: a-c: tantalum; d: Palladium; e: Niobium; f: Nickel. Reproduced with permission ${ }^{[329]}$ Copyright 2015, The Royal Society of Chemistry. (B) Corresponding cyclic voltammograms displaying characteristic current peaks for dopamine note that fibers without CNS do not reveal a dopamine signal. (C) A microfabricated implantable shank that displays multiple platinum electrodes functionalized with enzymes for detecting lactate and glucose (important metabolic markers). Reproduced with permission. ${ }^{[330]}$ 


\section{WILEY-VCH}

Copyright 2015, Elsevier B.V. (D) A microfabricated shank with buried microchannels for microdialysis. The semipermeable membrane is made of deep reactive ion-etched porous anodic alumina ${ }^{[139]}$, allows for an integrated membrane as opposed to a secondary polymeric coating prone to delamination. (E) A microfluidic circuitry for perfusing the interstitial space with artificial cerebrospinal fluid and encapsulating the dialysate via mineral oil-based droplet generator. The droplets are interrogated fluorescently downstream. Reproduced with permission $^{[143]}$. Copyright 2015, The Royal Society of Chemistry (F) A "chemistrode" for delivering soluble factors to theoretically modulate a region and sample from the same region for further downstream analysis ${ }^{[331]}$. Copyright 2008, National Academy of Sciences 


\section{WILEY-VCH}
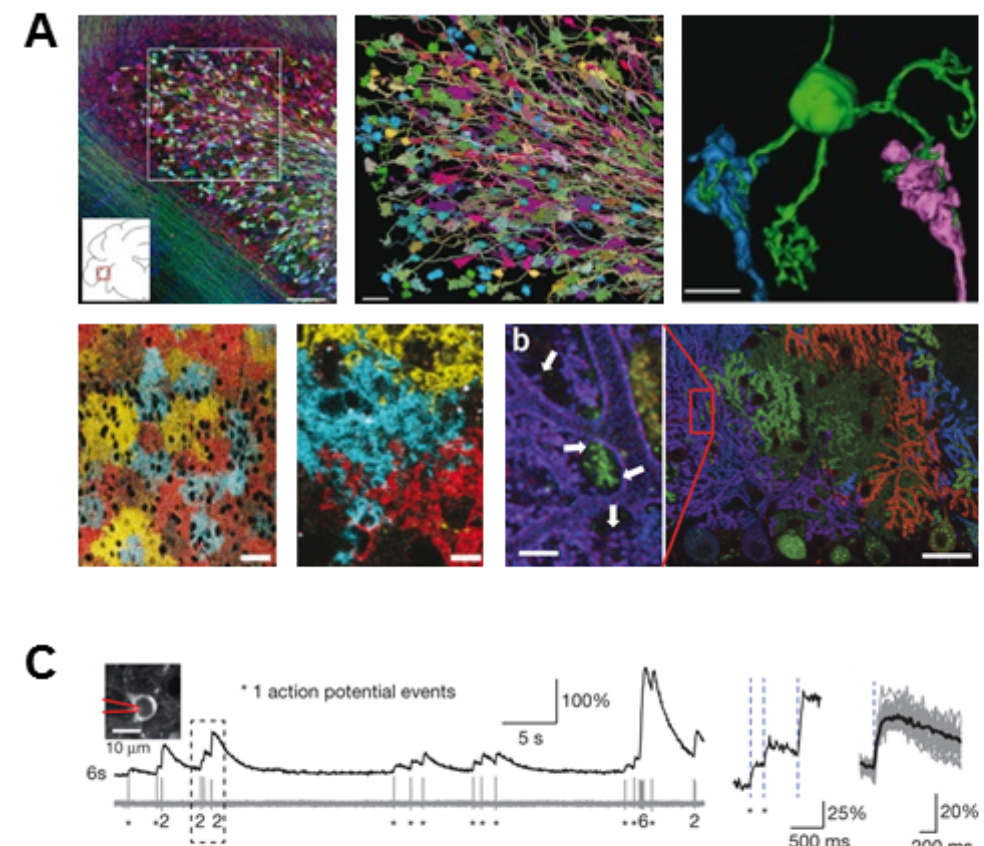

C
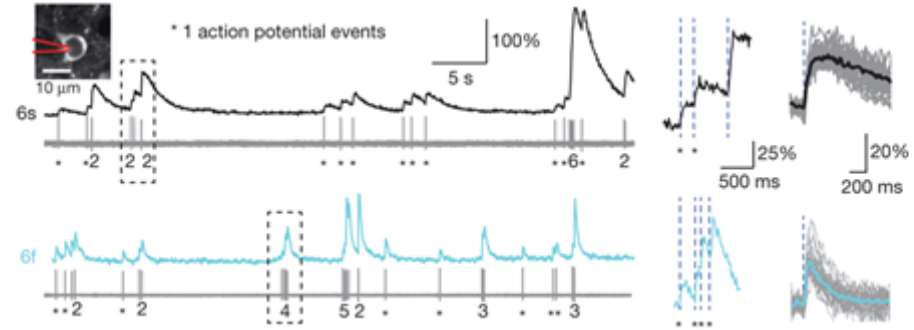
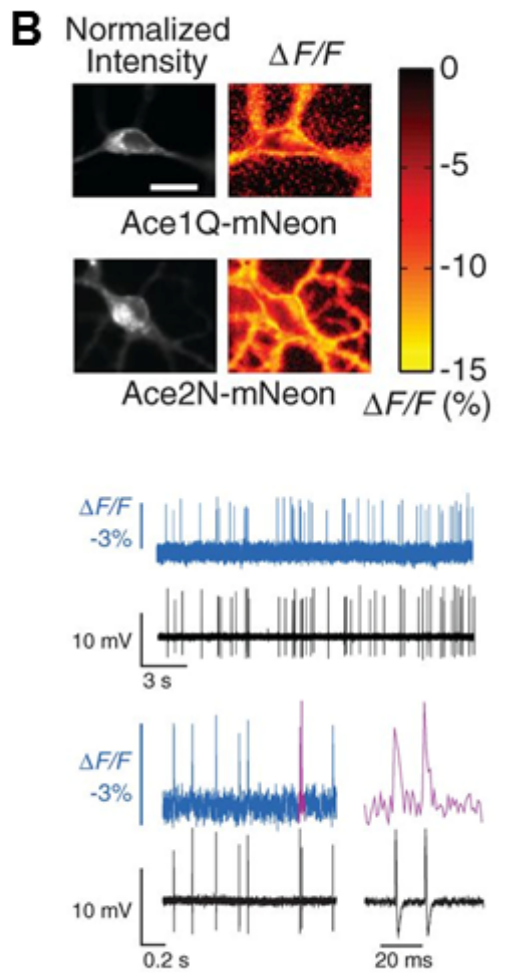

Figure 7. Examples of optical monitoring technologies. (A) Fluorescence images of neural cells labeled with different combinations of fluorescent proteins using the 'Brainbow' strategy. Top row (left to right), image of the cerebellar flocculus of an adult mouse taken using confocal sections. 3D digital reconstruction of the outlined region for circuit mapping. 3D reconstruction highlighting the connections between different neurons ${ }^{[161]}$. Reproduced with permission. Copyright 2007, Nature Publishing Group. Bottom row (left to right), Image of astrocytes labeled using the 'Brainbow' strategy showing their tiled structure ${ }^{[161]}$. Reproduced with permission. Copyright 2007, Nature Publishing Group. Image from the Brainbow 3.1 line of transgenic mice that allows for the visualization of fine processes through the expression of modified XFPs that were better trafficked to the axons and dendrites ${ }^{[162]}$. Reproduced with permission. Copyright 2013, Nature Publishing Group. (B) (Top) Example of the fluorescence signal from the GEVIs Ace1Q-mNeon and Ace2N-mNeon. (Bottom) Comparison of concurrent optical (blue) and electrophysiological (black) signals from in vivo recordings ${ }^{[332]}$. Reproduced with permission. Copyright 2015, The American Association for the Advancement of Science. (C) Comparing the fluorescence response of the GECIs GCaMP6s (top) and GCaMP6s (bottom) to loose seal, cell-attached recordings in the visual cortex ${ }^{[170]}$. Reproduced with permission. Copyright 2013, Nature Publishing Group. 
WILEY-VCH

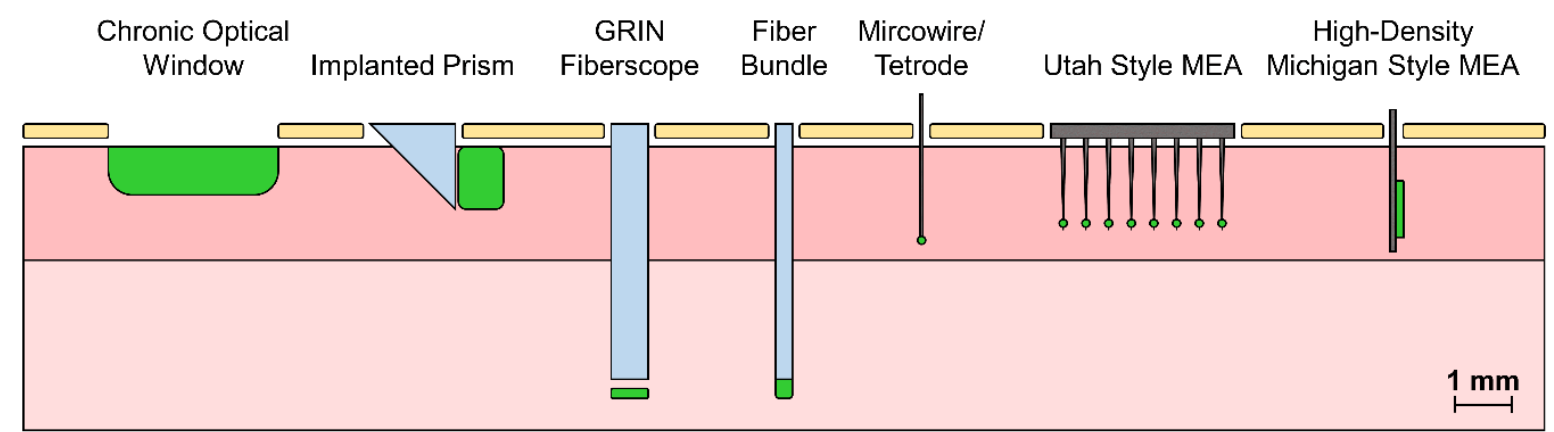

Figure 8. Representation of the volume of tissue (green) that can be monitored by optical interfaces and compared to some common electrical interfaces. The maximum distance from the interface that can be monitored is approximately $850 \mu \mathrm{m}$ for chronic optical windows ${ }^{[180]}$ and implanted prisms ${ }^{[185]}$. For the GRIN fiberscope and fiber bundle, the maximum image depth from the end of the fiber is approximately $340 \mu \mathrm{m}^{[190-192]}$. As a comparison, electrical interfaces can monitor neurons up to $\sim 140 \mu \mathrm{m}$ from the interface ${ }^{[41]}$. 


\section{WILEY-VCH}

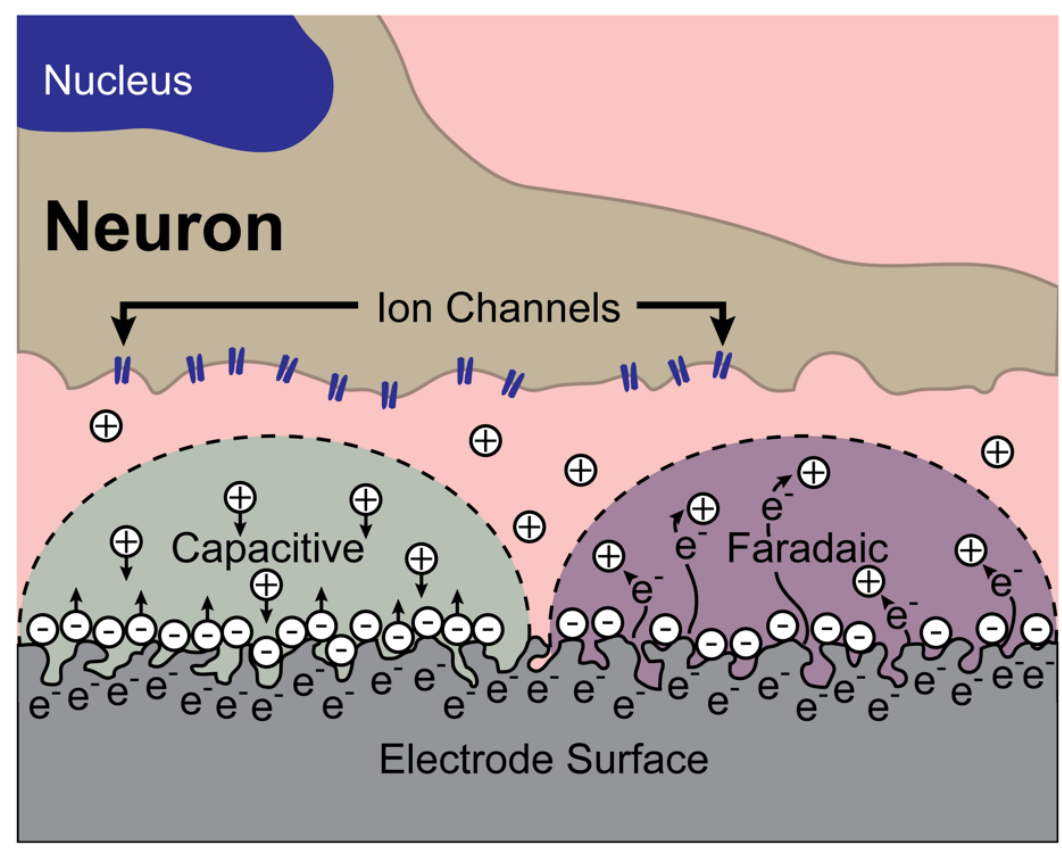

Figure 9. Equivalent circuit model of the electrical stimulation interface. During capacitive charge injection (green,) there are no chemical species produced or consumed, whereas during faradaic charge injection (purple), electrons are transferred between the electrode and surrounding media via redox reactions. Note: some materials behave in a pseudocapacitive manner (i.e., platinum and iridium oxide ${ }^{[4]}$ ) where their redox reactions are stable and no diffusion away from the material surface occurs. Since charge injection happens at the material surface, another major limiting factor is the electrochemically-addressable electrode surface area. 


\section{WILEY-VCH}

Improved Electrode Charge Injection Capacity via Nanostructured Materials
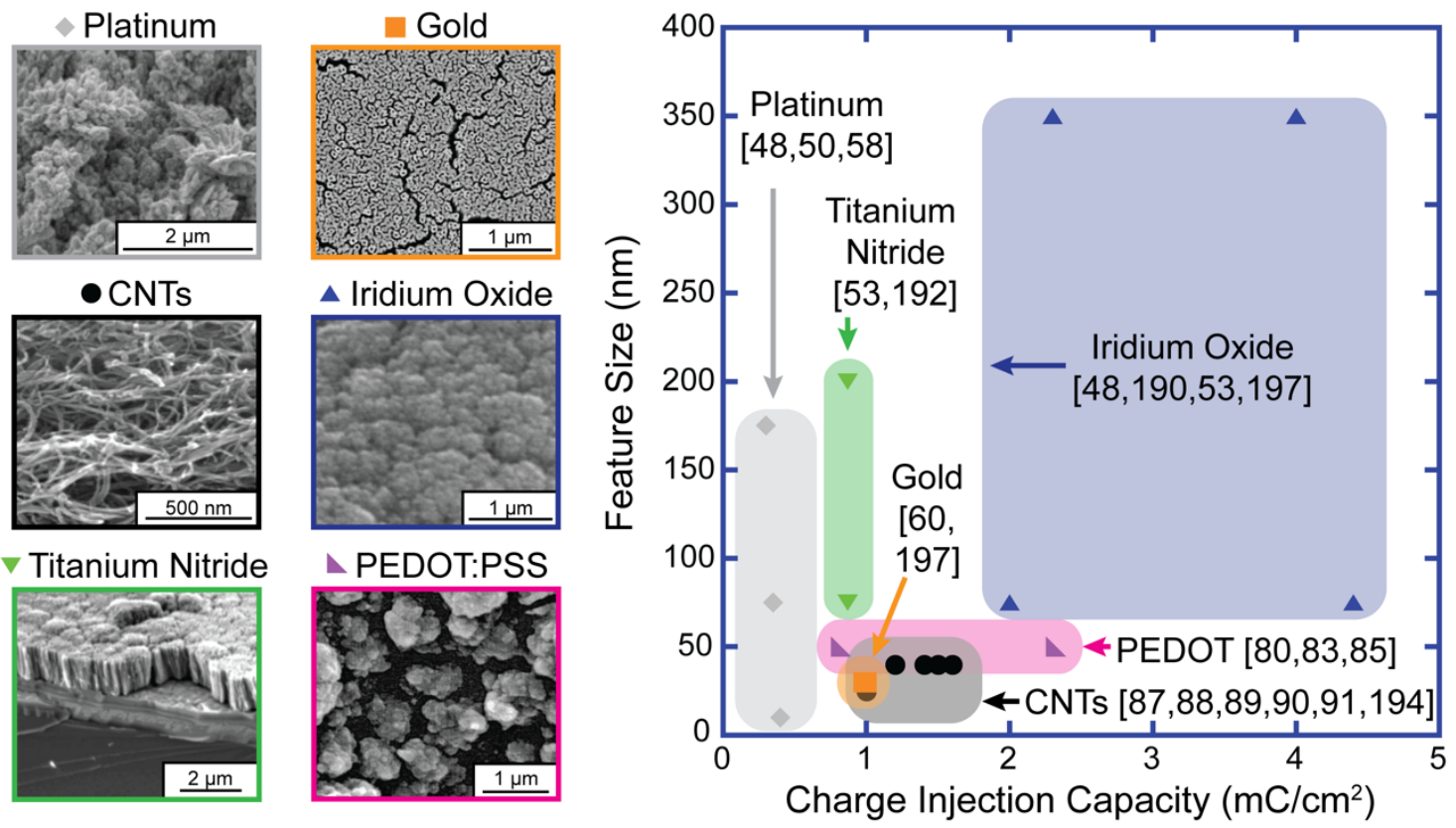

Figure 10. Examples of techniques to enhance electrical modulation of neural activity. (Left) Representative scanning electron micrographs of nanostructured materials (Platinum ${ }^{[327]}$ Reproduced per Creative Commons License, Gold ${ }^{[54]}$ Reproduced with permission. Copyright 2015, WILEY-VCH Verlag GmbH \& Co. KGaA. Carbon Nanotubes ${ }^{[328]}$ Reproduced per Creative Commons License, Iridium Oxide ${ }^{[52]}$ Reproduced with permission. Copyright 2004, IEEE. Titanium Nitride ${ }^{[53]}$ Reproduced with permission. Copyright 2002, IEEE. PEDOT:PSS ${ }^{[82]}$ Reproduced with permission, Copyright 2003, Elsevier B.V.). (Right) Representative graph illustrating the relationship between nanoscale feature size (estimated from scanning electron micrographs) and electrode charge injection limits reported in literature for each material. Overall, regardless of feature size, iridium oxide exhibits significantly higher charge injection capacity than the other materials reviewed. 


\section{WILEY-VCH}

A NEUROMODULATOR RELEASE VIA MICROFLUIDICS

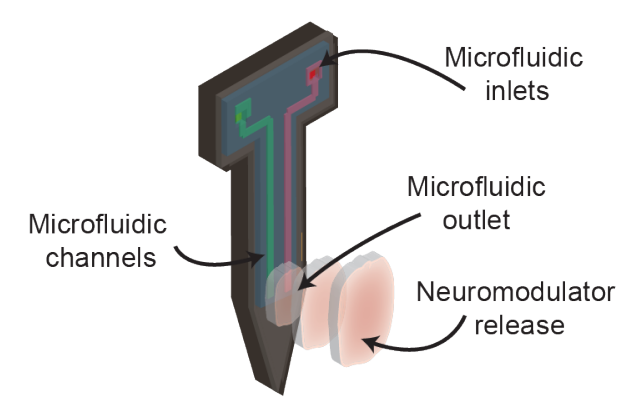

B NEUROMODULATOR RELEASE FROM COATINGS

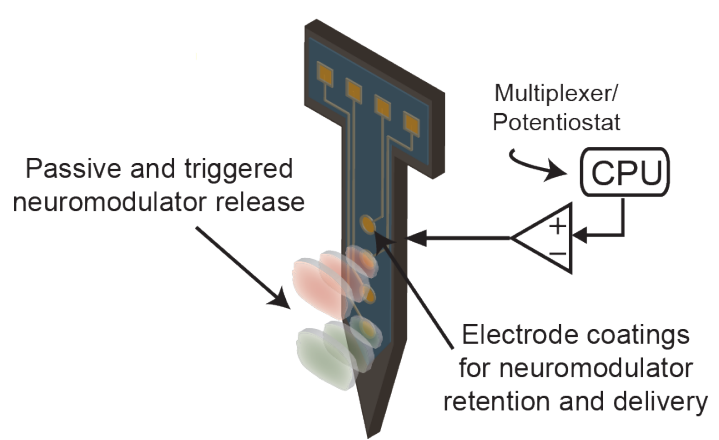

Figure 11. Schematic illustration of chemical interfaces for modulating neural activity. (A) A microfabricated shank with buried microchannels can deliver soluble factors via convective fluidic transport. (B) A microfabricated shank, where the electrodes are composed of or coated with materials that can retain neuromodulators and either release them. The release step can simply be passive diffusive efflux of molecules or it can be triggered by various stimuli (e.g., temperature, $\mathrm{pH}$ ). Electrically-triggered release allows for on demand delivery of neuromodulators. 


\section{WILEY-VCH}
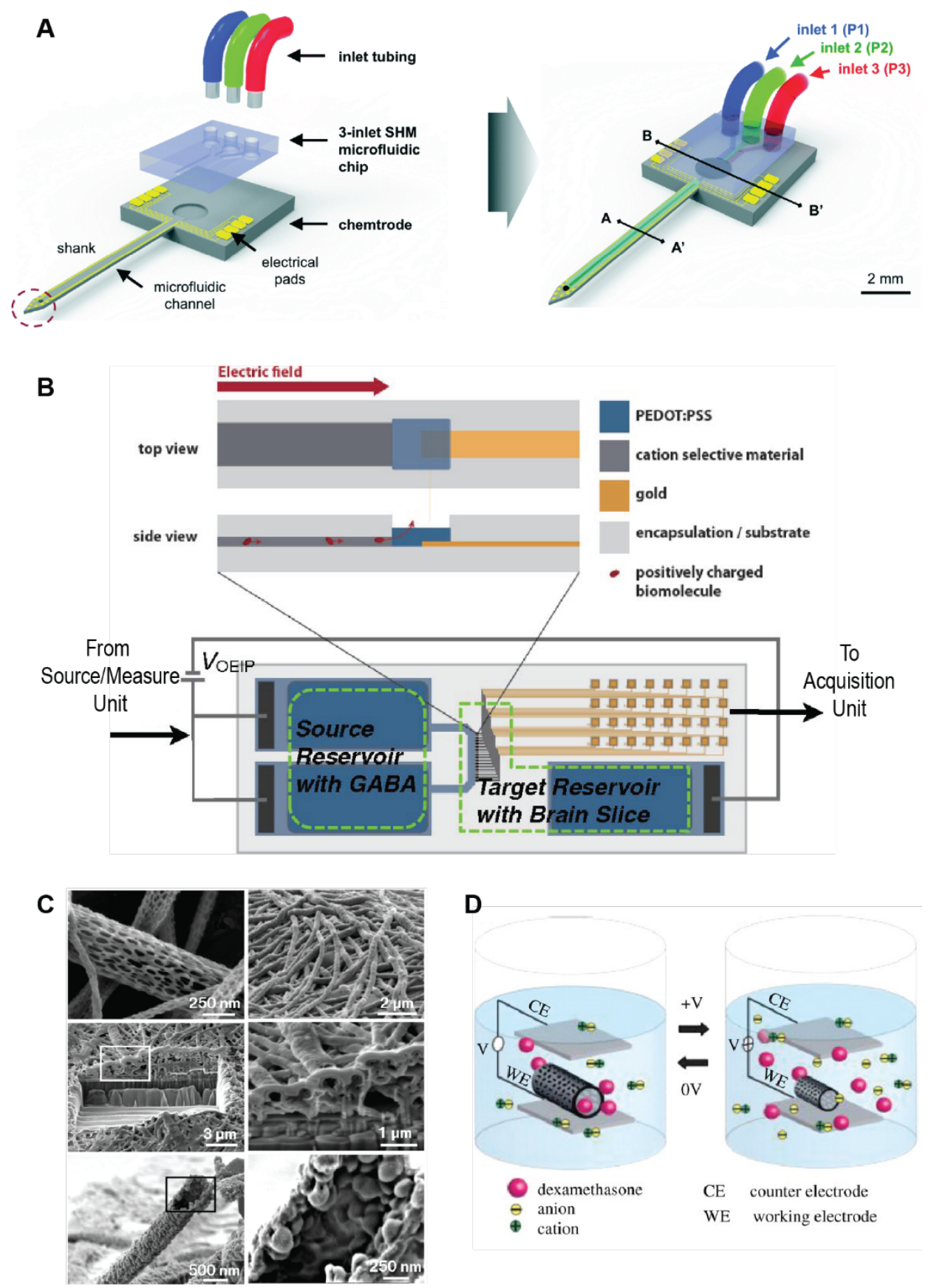

Figure 12. Examples of chemical modulation technologies. (A) A microfabricated shank with multiple microfluidic channels and corresponding release orifices. The electrical contacts on the same shank allow for electrophysiological recordings during fluidic delivery ${ }^{[219]}$. Reproduced with permission. Copyright 2015, The Royal Society of Chemistry (B) A device for electrically-triggered delivery of GABA, an inhibitory neuromodulator, and simultaneous monitoring of electrophysiological activity at the same site ${ }^{[88]}$. Reproduced with permission. National Academy of Sciences (C) Scanning electron microscope images of a polymer nanotube for electrically-triggered delivery of dexamethasone (D) ${ }^{[238]}$. 


\section{WILEY-VCH}

A

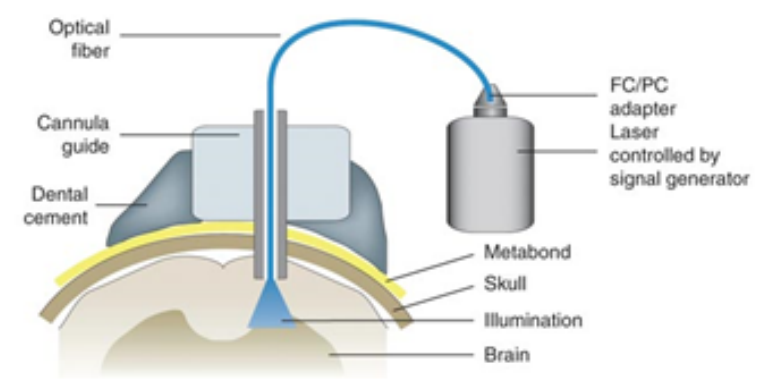

B
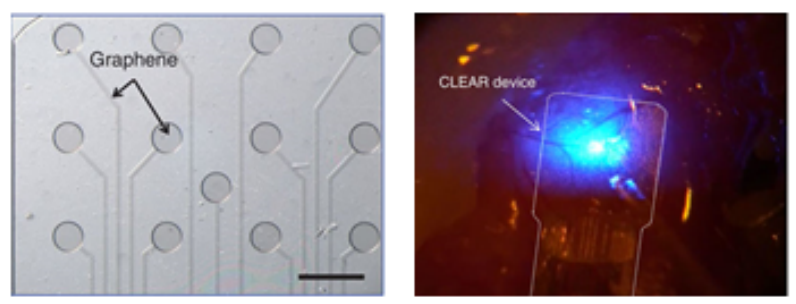

\section{C}
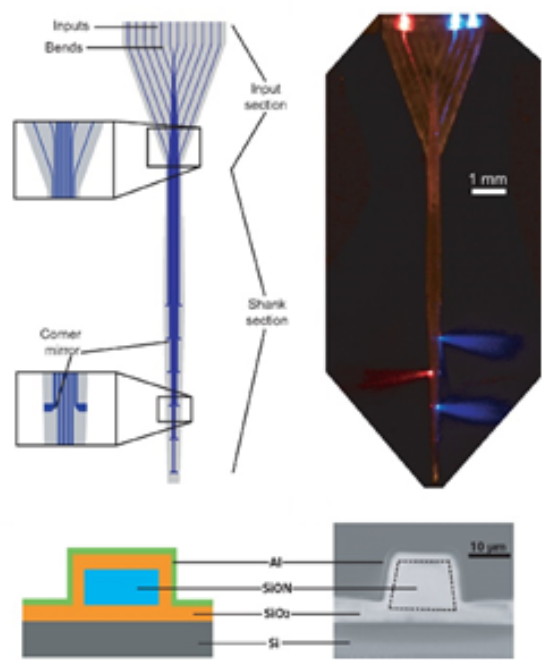

D
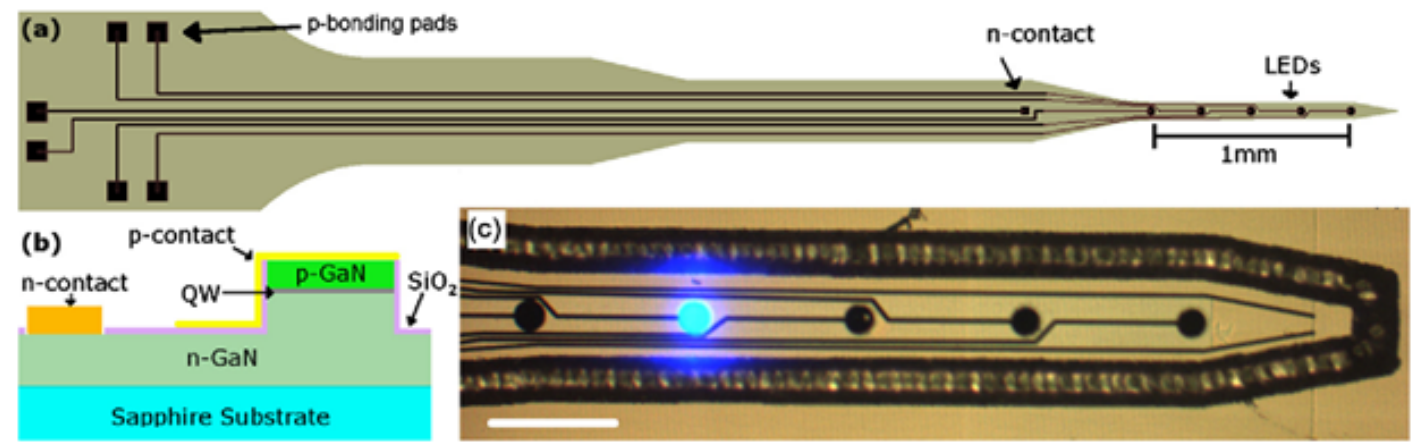

Figure 13. Examples of neural interfaces to provide optical stimulation. (A) Schematic of optogenetic stimulation using an optical fiber ${ }^{[333]}$. Reproduced with permission. Copyright 2010 , Nature Publishing Group. (B) Transparent ECoG array fabricated from parylene C and graphene ${ }^{[260]}$. Reproduced with permission. Copyright 2014, Nature Publishing Group. (C) (Top) Example of a multichannel waveguide for the independent optical stimulation of multiple sites. (Bottom) Fabrication schematic and cross-sectional image of an individual waveguide ${ }^{[263]}$. Published with permission. Copyright 2010, Optical Society of America. (D) Michigan-style microarray with micro-LEDs instead of recording electrodes ${ }^{[272]}$. Reproduced with permission. Copyright 2013, Optical Society of America. 


\section{WILEY-VCH}

A Biomaterial Development Library

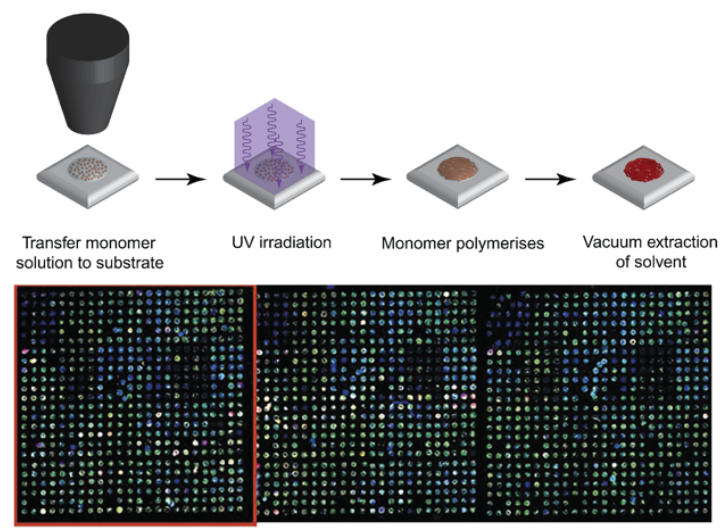

C Cell-Tissue Interaction Library
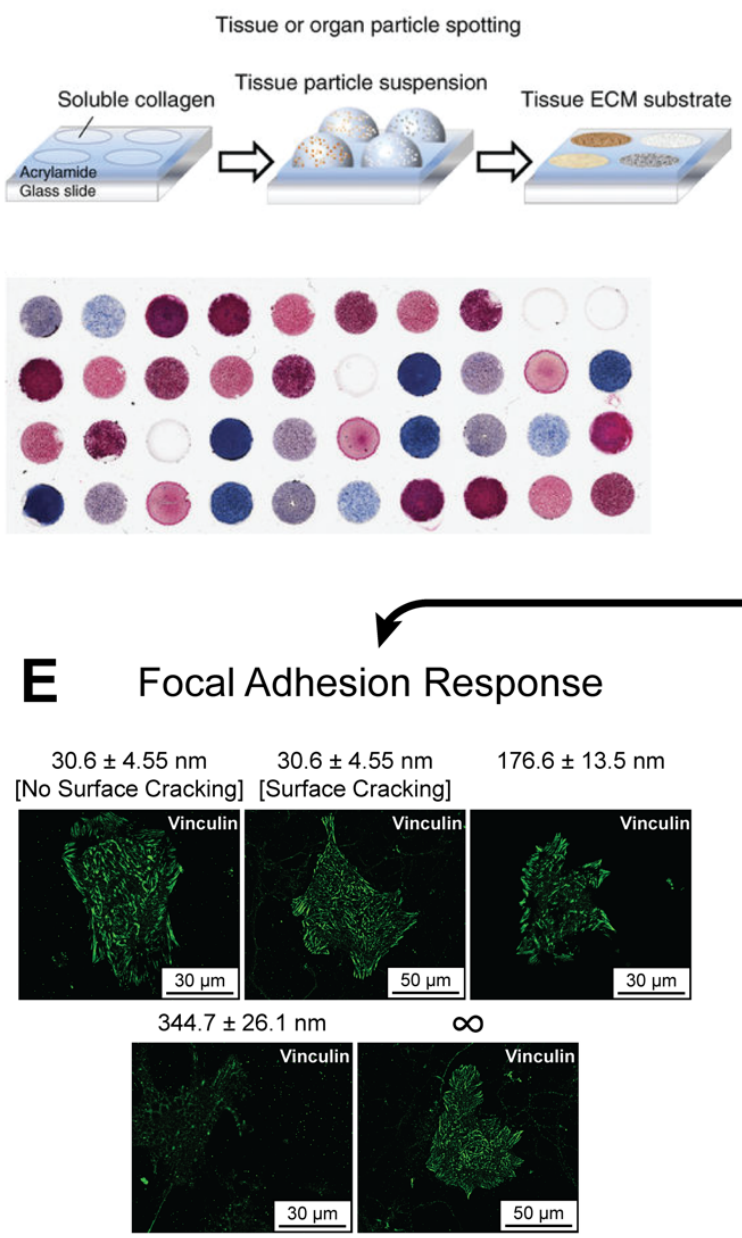

B Bacterial Adhesion Polymer Library
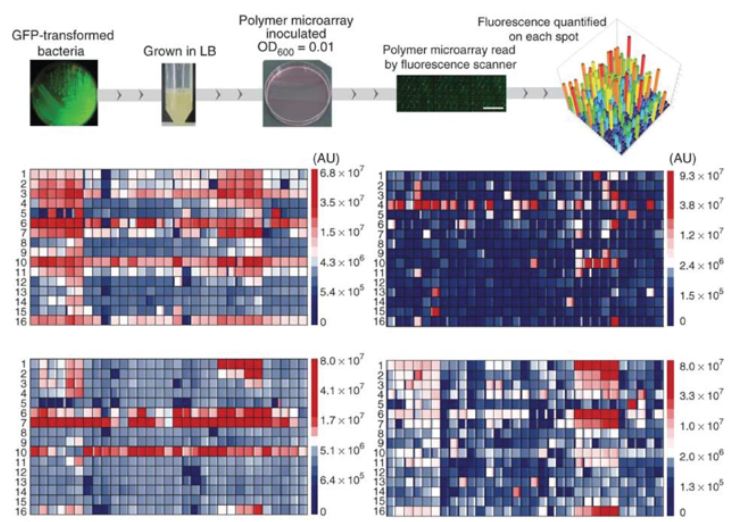

D Nanoporous Gold Material Library
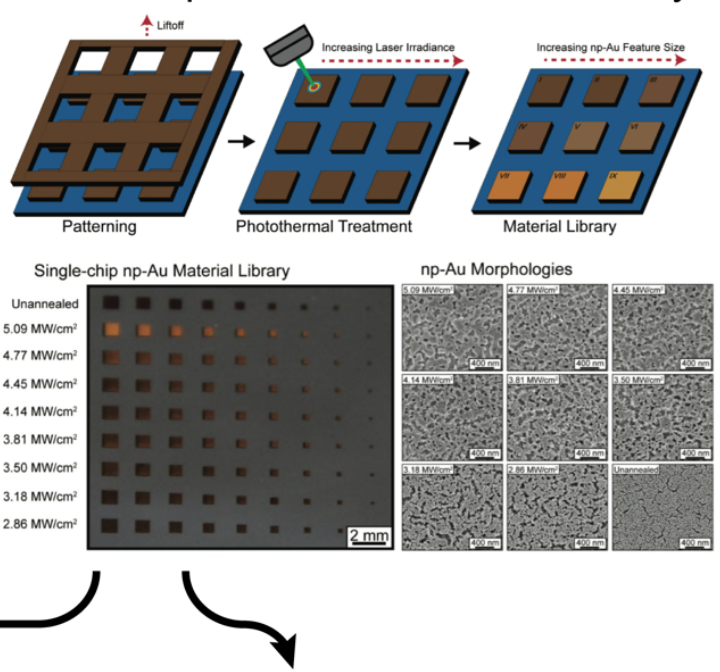

$\mathbf{F}$

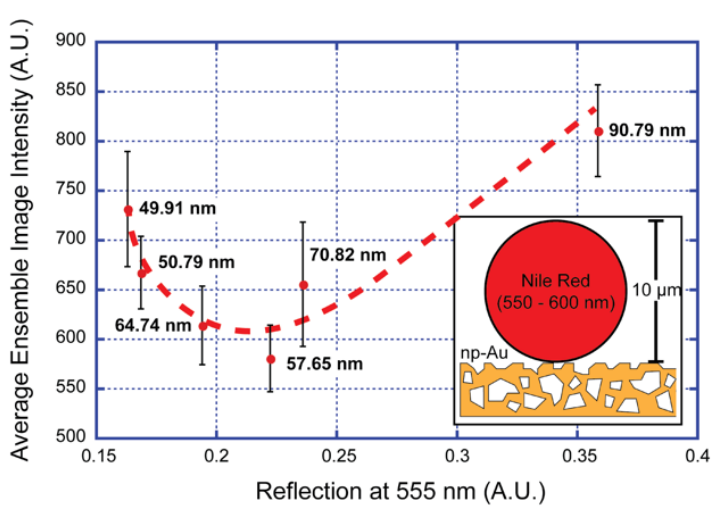

Figure 14. Representative material libraries for high-throughput screening. (A)

A biomaterial library for the study of new biomaterials for in vitro cell attachment, where the presence of green fluorescence identified differences in cellular reactivity to the biomaterial in question. ${ }^{[287]}$. Reproduced with permission. Copyright 2009, Elsevier Ltd. (B) A material library to study bacterial adhesion to polymers aimed at developing new antibacterial adhesive polymers, through quantifying heatmaps of fluorescence intensity of the GFP-transfected 


\section{WILEY-VCH}

bacteria $^{[290]}$. Reproduced with permission. Copyright 2012, Nature Publishing Group. (C) A combinatorial material library consisting of different tissue types. This library of tissues was used to study cell reactivity of certain cancers in a tissue type-dependent manner through a fluorescence readout ${ }^{[293]}$. Reproduced with permission. Copyright 2015, Nature Publishing Group. (D) A material library of different np-Au feature sizes. Through patterning and laser processing libraries with a large number of morphologies can be created ${ }^{[295]}$. Reproduced with permission. Copyright 2016, The Royal Society of Chemistry. Direct applications of the nanoporous gold material libraries. These libraries have been used to (E) study the cell typedependent adhesion to nanoporous gold as a function of feature size ${ }^{[54]}$. Reproduced with permission. Copyright 2016, Wiley-VCH Verlag GmbH \& Co. KGaA., as well as (F) study how fluorescence intensity varies depending on reflectivity and surface effects stemming from changes in nanoporous gold feature size ${ }^{[296]}$. 
WILEY-VCH

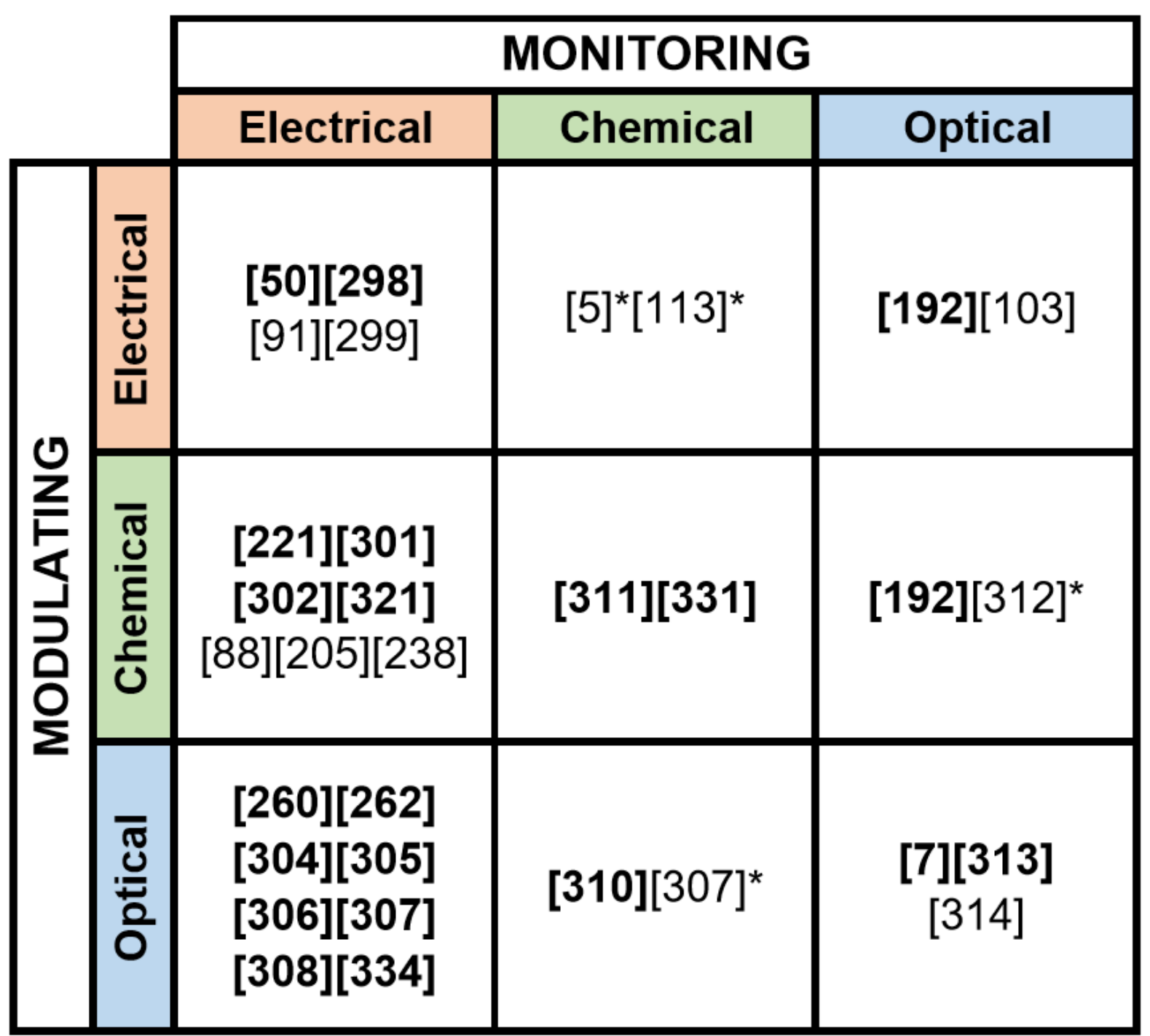

Figure 15. Combinations of modalities on a single footprint. Bolded references have had both the monitoring and modulating capabilities of the device demonstrated in vivo. Asterisks indicate references that contain devices with multifunctional capabilities that were not explicitly examined during the study. 


\section{WILEY-VCH}

Electrical Monitoring/Electrical Modulation

A
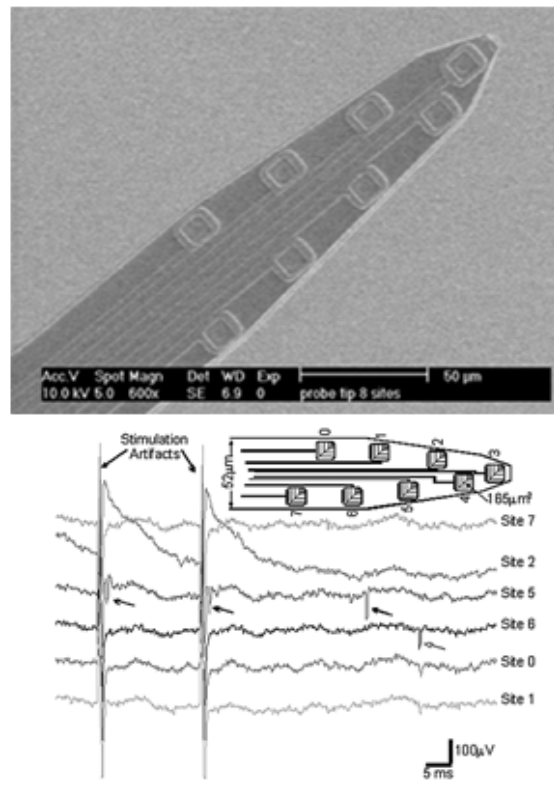

Electrical Monitoring/Chemical Modulation

B
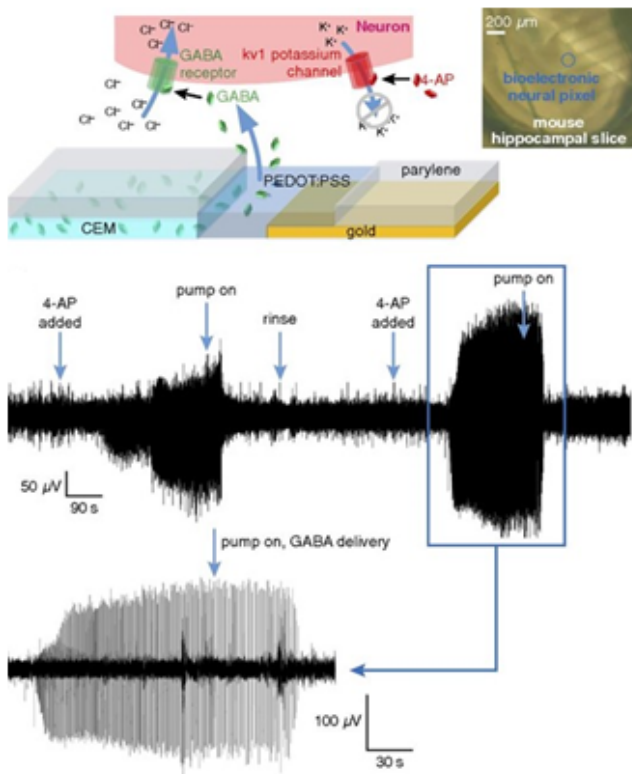

Electrical Monitoring/Optical \& Chemical Modulation
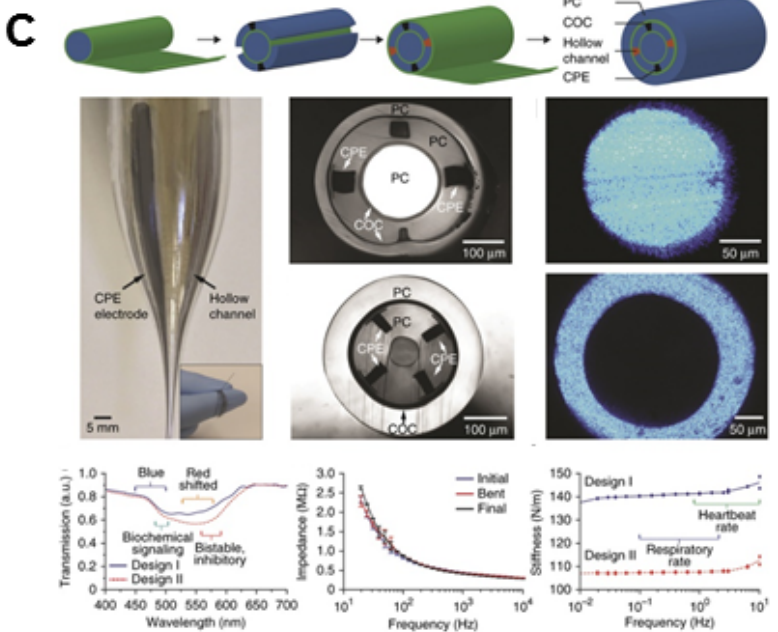

Electrical Monitoring/Optical Modulation

D

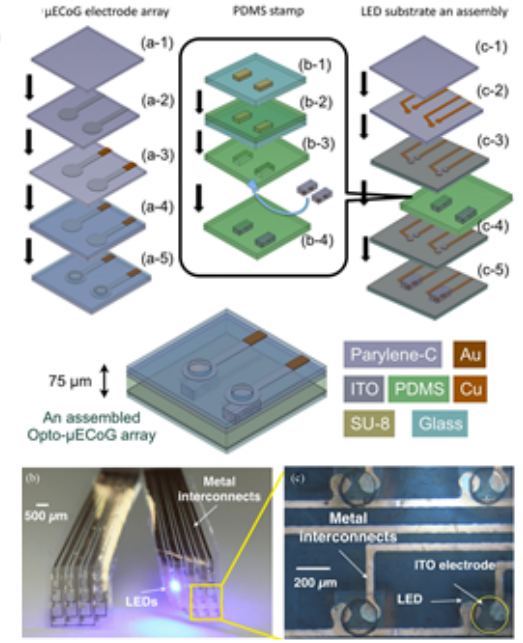

Electrical Monitoring/Chemical Modulation

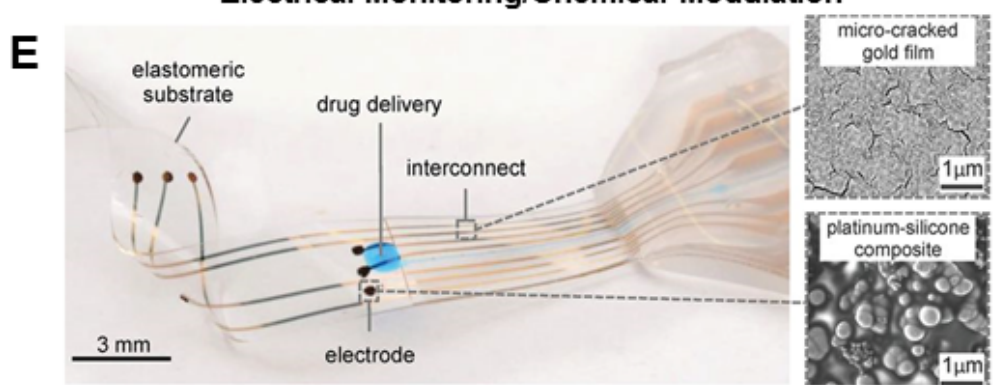

Figure 16. Examples of multifunctional neural devices and interfaces. (A) (Top) SEM image of a combined electrical stimulating and recording interface with iridium electrodes. (Bottom) Recording artifacts directly following the electrical stimulation ${ }^{[298]}$. Reproduced with permission. Copyright 2005, IEEE. (B) (Top) Schematic and image of the "bioelectronic neural pixel". Organic electronic ion pumps are integrated into PEDOT:PSS recording electrodes, and release the inhibitory neurotransmitter GABA when a current is applied. (Bottom) Epileptiform activity recorded prior to and following the release of GABA from the interface, demonstrating the ability of the interface to electrically record and chemically modulate simultaneously ${ }^{[88]}$. 


\section{WILEY-VCH}

Reproduced with permission. National Academy of Science. (C) (Top) Multifunctional interface capable of electrical recording and chemical and optical modulation fabricated using a thermal drawing process using polymeric materials. A schematic of the different polymer layers is shown and (Middle) images of the drawn fiber along with cross-sectional images showing the different polymers and optical transmission. (Bottom) The optical transmission through the fiber, impedance of the recording electrodes and mechanical stiffness of fiber is shown ${ }^{[304]}$. Reproduced with permission. Copyright 2015, Nature Publishing Group. (D) (Top) Fabrication schematic of an array of multifunctional interfaces consisting of a transparent ITO electrode and $\mu$ LED. (Bottom) Images demonstrating the capabilities of the device ${ }^{[334]}$. Reproduced with permission. Copyright 2013, IEEE. (E) Example of a flexible MEA with an integrated microfluidic channel for chemical modulation ${ }^{[321]}$. Reproduced with permission. Copyright 2015, The American Association for the Advancement of Science. 


\section{WILEY-VCH}

\section{Biographies}

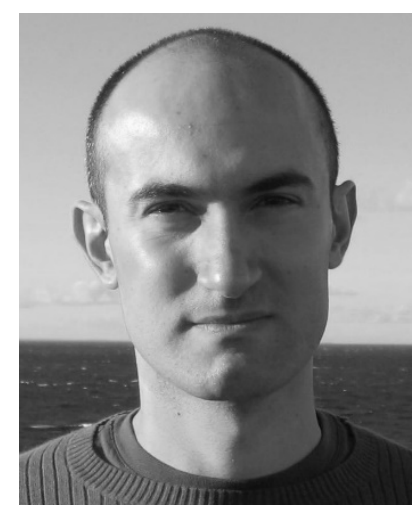

Erkin Şeker is an associate professor in the Department of Electrical and Computer Engineering at University of California - Davis (UC Davis) in 2011. He received his PhD degree in Electrical Engineering from University of Virginia (UVA) in 2007. He was a postdoctoral fellow in the Chemistry Department at UVA between 2007-2009 and was a postdoctoral research associate at the Center for Engineering in Medicine at Harvard Medical School between 2009-2011. He leads the Multifunctional Nanoporous Metals group that studies nanostructure-property relationships in the context of biomedical applications.

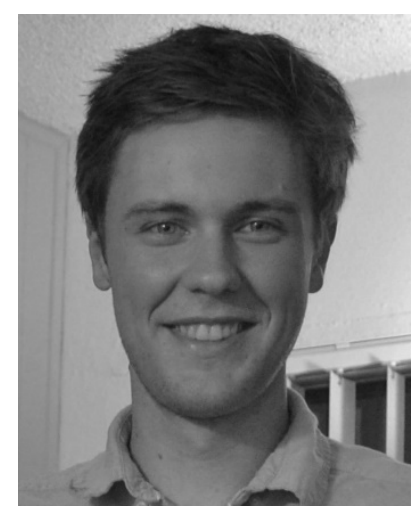

Christopher Chapman is a postdoctoral research associate in the Electrical Impedance Tomography group at University College London. He received his $\mathrm{PhD}$ degree in Biomedical Engineering from the University of California - Davis in 2017 under the mentorship of Prof. Erkin Seker. Prior, he obtained B.S. degrees in Biomedical Engineering and Mechanical Engineering from North Carolina State University in 2012.

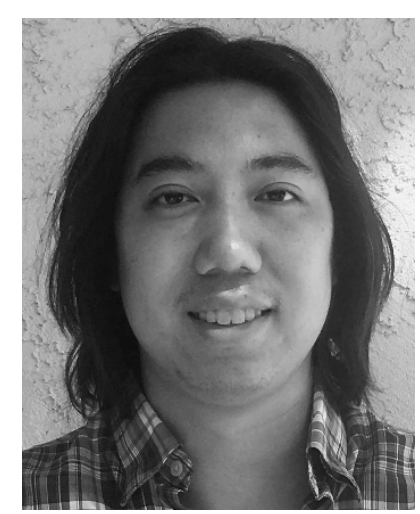

Noah Goshi is a Ph.D. student in the Multifunctional Nanoporous Metals group at the University of California - Davis. Prior, he obtained his M.S. in Bioengineering from San Diego 


\section{WILEY-VCH}

State University in 2016 and a B.S. in Bioengineering: Biotechnology from the University of California - San Diego in 2011. 


\section{WILEY-VCH}

The capability to monitor and modulate neural activity in a closed-loop fashion is essential for studying the nervous system and managing neurological disorders. This feature article introduces the prominent technologies for interfacing with the nervous system via electrical, chemical and optical means, followed by a discussion of opportunities and challenges in developing multifunctional interfaces that embody the three modalities.

\section{Multifunctional Neural Interfaces}

Christopher A. R. Chapman, Noah Goshi, Erkin Seker*

Multifunctional Neural Interfaces for Closed-Loop Control of Neural Activity

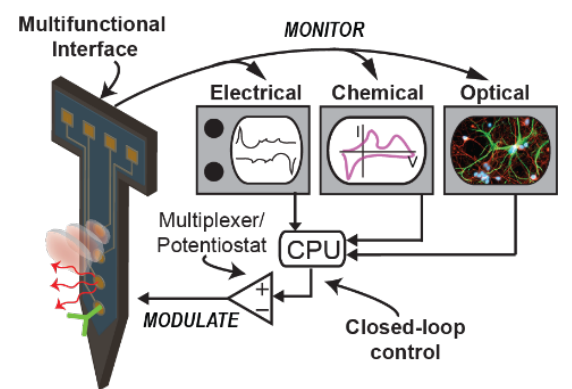




\section{WILEY-VCH}

Copyright WILEY-VCH Verlag GmbH \& Co. KGaA, 69469 Weinheim, Germany, 2016. 